\title{
DIAGNOSTICS FOR STEADY STATE PLASMAS
}

\author{
H.-J. Hartfuss, R. Koenig, A. Werner \\ Max-Planck-Institut fuer Plasmaphysik, EURATOM Association \\ 17491 Greifswald, Germany
}

\begin{abstract}
Problems related to the development of diagnostics for steady state fusion plasma experiments are being discussed. The paper concentrates on those necessities already appearing in nowadays non-burning plasma fusion experiments when extending pulse lengths beyond $10 \mathrm{~s}$, i.e. thermal load, erosion, deposition, and long-time signal integration in magnetic diagnostics. Problems arising from high power ECRH under conditions of incomplete absorption are outlined. Individual standard diagnostic systems are discussed to identify their specific problems as well as the opportunities connected with long pulse operation. Burning plasma experiments characterized by intense $\mathrm{n}$ - and $\mathrm{Z}$-radiation are briefly reviewed for reasons of completeness, dealing with radiation induced processes in windows, fibres, cables, and mirrors. Methods of data handling, real time monitoring, and plasma control are outlined.
\end{abstract}




\section{Index}

1. Introduction

2. Real time monitoring and control

3. Diagnostics to keep plasma performance

4. Diagnostics to permit device protection

5. General considerations for long pulse diagnostics

5.1 Difficulties associated with long pulses

5.2 Opportunities associated with long pulses

5.3 Data acquisition

6. Non-absorbed ECRH stray radiation

6.1 Expected stray radiation level

6.2 Specific effects

6.3 Non-specific effects

7. Problems and considerations for standard diagnostics

7.1 Magnetics

7.2 Probes

7.3 Spectroscopy

7.4 Thermography

7.5 Bolometry

7.6 Thomson scattering

7.7 Microwave diagnostics

7.8 Particle diagnostics

7.9 Divertor diagnostics

8. Radiation effects in burning plasma experiments

9. Plasma facing optical components

9.1 Windows

9.1.1 Thermo-mechanical effects

9.1.2 Radiation effects in windows

9.1.3 Contamination of diagnostic windows

9.1.4 Mirror and window cleaning techniques

9.2 Mirrors

9.2.1 Radiation

9.2.2 Erosion

9.2.3 Coating

10. Fibres

9.2.4 Long pulse experiments

11. Cables

12. Magnetic measurements

12.1 Plasma parameters and sensor requirements

12.1.1 Equilibrium

12.1.2 Fluctuations

12.2 Sensor design

12.3 The long pulse integration problem

12.4 Alternative concepts

12.5 Automated analysis

13. Summary and conclusions

References 


\section{Introduction}

Amongst present day fusion devices operating under relevant parameters, an increasing number of facilities exists, dedicated for long pulse or quasi steady state operation. Both tokamak and stellarator experiments like Tore Supra (TS), the Super Conducting Japan Tokamak (JT-60-SC), the Korean Superconducting Tokamak Advanced Research (KSTAR), the Chinese Superconducting Tokamak HT7-U, the Indian Steady State Tokamak (SST-1), the Large Helical Device (LHD), and the Wendelstein 7X (W7-X) with confining fields generated with superconducting coils are being operated or are under construction. They are preparing in many different aspects the tools for the successful operation of the burning plasma experiments (BPX) to come by contributing to the development in a variety of physics and technology fields. The internationally agreed ITER device is being planned, it will be constructed and operated to demonstrate and to understand the behaviour of plasmas dominated by $\square$-particle heating and to learn to operate the device, its instrumentation and the plasma diagnostic system under the harsh environmental conditions of a burning plasma [1-3]. The high level of neutron- and 7 -radiation will be one of the major challenges. The strong radiation will primarily affect all plasma facing components and the structural elements of the machine. Careful choice of all in-vessel materials and their chemical element composition is highly demanded.

Radiation will impose for the diagnostic systems in addition to the broadened parameter range to be covered and the necessity of providing new measuring techniques in that respect extreme challenges in particular for the design of the plasma diagnostics front ends. Windows and fibres as used in nowadays fusion experiments are subject of radiation induced absorption (RAI) and radiation induced emission (RIE, radio-luminescence) which strongly affect their optical properties. Refractive components can not be used anymore in the way they are used so far in close vicinity to the plasma. All diagnostics using UV, visible, or IR radiation must view the plasma via mirrors, which have to be mounted in a mirror-relay type of arrangement in a port-integrated labyrinth for sufficient shielding of subsequent components. However, neutron-bombardment changes the surface quality of metallic mirrors as well and might severely deteriorate their quality due to swelling of the structural and surface materials. Cables installed within the vacuum vessel are affected by the radiation causing their quality to deteriorate. Voltages and currents are being generated along the cable, introducing significant errors in magnetic measurement.

While the effects listed so far will only be present under BPX conditions, additional effects must be considered which pose severe constraints already for present day experimental devices operated under long pulse quasi steady state, fusion relevant conditions. High power heating as conducted for example in the TS tokamak, the LHD helical system, and the future W7-X stellarator and the KSTAR tokamak devices results in strong thermal loading of all plasma facing components (PFC) mainly owing to UV- and VUV-radiation. It demands for their active cooling on the one hand and on high reliable monitoring systems on the other. Cooling demands impose in particular severe problems to optical windows. In case continuous heating power is provided on a MW-level by wave heating, additional thermal loading and/or specific interactions of in-vessel components with non-absorbed background stray radiation of ICRH, LH, and in particular ECRH might impact their functionality.

All plasma facing optical components (PFOC), windows and mirrors are being degraded by surface modifications and erosion as a result of the continuous bombardment by energetic 
neutral particles resulting from charge exchange processes $(\mathrm{CX})$ in the plasma. In addition surface coating of mirrors and windows with re-deposited vessel wall material might change the transmission characteristics in a significant way demanding for cleaning and in-situ calibration techniques. Water cooled shutters need to be developed to protect PFOCs under wall conditioning scenarios and other operation conditions where optical viewing is not necessarily demanded.

It is mandatory to extend the operation of existing devices to long pulses and quasi steady state operation. In the frame of the tokamak concept with the plasma current generated by induction, scenarios have been established with zero loop voltage with driven currents fully sustained by non-inductive wave launching and/or NBI techniques to create and control the current profiles to avoid MHD activity or establish improved confinement regimes due to internal transport barrier formation. Long pulse advanced scenarios governing high confinement, exploring the $\square$ - and density limits, with reliable disruption avoidance, with impurity and particle transport control amongst many others must be further investigated and established. Studies of processes appearing on the longer time scales, for example current diffusion and particle retention in the wall must be conducted and technological opportunities and constraints connected with the development and safe operation of high heat flux resistant plasma facing components be explored since they are the important challenges addressing the future fusion reactor. Since time scales of various in-vessel components extend into the several hundreds of seconds range, pulse duration of up to $1000 \mathrm{~s}$ to reach total thermalisation of all in-vessel components must be envisaged. Tokamak long pulse discharges have successfully been conducted lasting more than 6 minutes in Tore Supra [4, 5], about $60 \mathrm{~s}$ in JT-60U [6], and $5 \mathrm{~h}$ and $16 \mathrm{~min}$. in TRIAM-1M [7].

Stellarators face similar problems as the tokamaks, however, since they are disruption-free, intrinsically steady state devices, once the confining field is set up by superconducting coils, no active position stabilization is needed. LHD successfully conducted long pulse high power discharges with 1.29 GJ total energy input, lasting more than half an hour [8]. Operation of stellarators might nevertheless be limited by incomplete particle and impurity control, and depend on the achieved level of controlled power exhaust, and the successful solution of the various technically oriented problems connected with thermal loading, erosion, and coating as mentioned before.

Since the development of the stellarator concept is lagging behind the tokamaks by one machine generation, further concept improvements need reliable data gained under quasistationary conditions. The W7-X experiment for example is a concept test for properties of reactor relevant plasmas in advanced stellarators. W7-X will be heated by continuous ECRH and pulsed NBI and ICRH. The macroscopic MHD timescale to establish equilibrium of confinement with respect to bootstrap current and current density distribution is about $10 \mathrm{~s}$. This means confinement studies already demand for pulse lengths of $100 \mathrm{~s}$. Time constants related to the gas inventory of the wall and impurity flux due to plasma-wall interaction are of the order of minutes. Divertor experiments and divertor component developments have to cope with these conditions and demand for the long pulses.

Two additional problems are solely connected with diagnosing steady state devices. One is dealing with the usability and operation of the important magnetic diagnostics in particular with the drift-free long-time integration of the induced signals of the inductance loops. The other problem is intelligent handling of the huge amount of diagnostics data, a significant part of which must be available in real-time for monitoring purposes to safely operate long pulse discharges. 
Given this frame, problems and demands for steady state plasma diagnostics are basically manifold. However, they can be divided into two main categories: Problems connected with long pulse operation in general and those connected with intense n-, and Gradiation in a future BPX. Of course, BPX diagnostics have to deal with both [9]. This paper will, however, concentrate on the first category. The radiation problems will be covered only briefly in this article. Excellent papers exist on this subject. Almost exhaustive information has been collected and prepared in the frame of ITER diagnostics definition and related preparatory work [1-3, 10-17]. Based on these papers a summarising discussion of the main problems is included for completeness. Problems connected with new measuring demands in BPX will not be reviewed: the determination of the birth profile and the distribution of $\square$-particles in the burning plasma for example or the necessary consideration of relativistic effects becoming dominant at electron temperatures as expected in ITER. In later operation phases of a BPX the diagnostic orientation might moreover change. Components tests at high neutron flux and fluence might become a main goal. The number and the sophistication of the diagnostic systems of the set might then be reduced and might change in favour of more technologically oriented measuring systems.

This paper tries to complement those mentioned by concentrating on the long pulse specification of the key standard diagnostics. Problems and development needs of these diagnostics are briefly discussed in separate paragraphs. On the basis of the experience gained developing W7-X diagnostics, the paper reviews in particular the thermal load and the coating of front-end windows, problems associated with non-absorbed microwave stray-radiation, and the long-pulse integrator development for magnetic diagnostics. Diagnostic opportunities associated with long pulses are included as well.

\section{Real time monitoring and control}

Beside plasma stabilization and real time plasma boundary control of tokamak plasmas, all steady state fusion experiments need excellent capabilities to analyze and to control the plasma. Automated analysis and monitoring of diagnostic data is mandatory in long pulses and since manual analysis of the huge data sets is anyhow no longer feasible at discharge durations of the order of 1000 s or more and therefore can only be selective. Automated analysis and monitoring is essential for a safe machine operation, since in steady state machines first wall components can be easily overloaded due to the missing thermal inertia effects.

Basically, a discharge can be operated passively, i.e. by pre-programmed machine control, or actively, i.e. the online analyses force reaction of the device control system. In tokamaks, low latency real time feedback loops for vertical stabilization and plasma shape control are used routinely via particular shape controllers. Beside this type of active control, in passive mode all other experiment settings are adjusted before the pre-programmed plasma start aiming at the discharge evolving into the steady state of interest, e.g. into an H-mode or a detached divertor plasma regime. Presumably, this approach will cost a lot of experimental time for the proper adjustment of the experimental settings and it is likely, that after several $L / R$ and plasma-wall interaction times the plasma state depends only weakly on the initial conditions. Active plasma control is also needed to establish advanced tokamak scenarios in long pulses. The needs for steady state tokamak control is summarized in [18].

In contrast to this, long pulse stellarators need also active plasma control, at least for accessing and stabilizing particular plasma operation regimes. Due to longer time scales 
involved, feed back and feed forward loops can be established making use of complex data analysis even under inclusion of predictive modelling. The latency depends on the complexity of the plasma parameters to be controlled or monitored. In W7-X plasmas for instance, the island divertor configuration is modified by the residual bootstrap current affecting the distance between X-Points and divertor targets. This parameter can be controlled by NBI or EC current drive on rather long time scales of the order of $10 \mathrm{~s}$, which are determined by current diffusion and L/R time constants. This latency allows rather complex data analysis including equilibrium reconstruction, divertor thermal load analysis and prediction of the required ECCD by ray tracing codes. In order to obtain a current control signal, predictive models for the current profile evolution have to be integrated as well [19].

A precondition for establishing this kind of plasma control is the automated data analysis. The daily experience in manual data analysis is that the information coming from a single diagnostic system is not accurate and reliable enough. In many cases the combination of different diagnostic data improves the information quality, even resolves contradictions and reveals systematic errors.

The integrated data analysis (IDA) allows an objective combination of all diagnostic data. It is based on the systematic combination of Bayesian statistical models of several (or even all) diagnostics, which include the modelling of uncertainties in a mathematically strict way [20]. Starting from the experimental data, the most probable plasma parameter set can be found that is in agreement with the data. This approach has become possible due to increased speed of modern computers, but presently the data analysis is still too slow for online data analysis. Thus, IDA is intended to be used for offline analysis.

The online data analysis, and consequently the data monitoring, has to be based on much simpler functions and approximations. Such rather simple functions may consist of analytical approximations or artificial neural networks and can be tested with regard to their suitability employing IDA results. Once the online analysis works reliably, real time variants may be derived from online analysis functions by further simplifications or implementation in dedicated hardware modules [21].

Real time monitoring requires reliable and steady state capable diagnostics. Tokamaks are well advanced and experience from JET with an outlook towards future steady state devices has been given recently [22]. For the development of real time capable diagnostics, a selection has to be made based on the availability for the whole discharge duration and whether the online analysis functions are reliably and fast enough to deliver responses within a certain latency time. In W7-X for instance, it is envisaged to get a suitable equilibrium reconstruction by function parametrization routines every $100 \mathrm{~ms}$. Consequently the magnetic diagnostic and the temperature and density profile diagnostics should run permanently at sufficiently high repetition rate. In particular the equilibrium information is essential for the mapping procedures in data analysis of other diagnostics enabling them to be used for monitoring and plasma control as well. For tokamaks, the reliability of magnetic sensors is of extreme importance and algorithms have to be developed, which can tolerate and detect loss of reasonable sensor signals. In case of ITER it has been reported that a 10-20\% loss of equilibrium sensor signals is believed to be tolerable [23].

\section{Diagnostics to keep plasma performance}

Steady state operation of tokamaks and stellarators requires, as discussed, continuous monitoring and feedback of a large number of physical quantities [24]. For tokamak control, 
measurements of the plasma shape and its position, of the loop voltage and the identification of modes are essential. Towards steady state a substantial fraction of current must be driven by non-inductive measures. Additional measurements are needed, and the tools need to be provided, to maintain plasma shear and shape at conditions for optimum confinement in advanced tokamak scenarios. Knowledge of the magnetic field structure and the current distribution is of fundamental importance for achieving optimum performance. Since the necessary measurements are difficult, equilibrium calculations are needed as discussed in the previous chapter. Under BPX conditions additional parameters become important like localization of $\square$-particle generation, their density as well as neutron emission profiles and related spectra. Generally diagnostics must provide conditions to sustain burn at a controlled fusion power level [14, 16, 25-27].

It is the aim to conduct future discharges in feedback with a variety of measurements. At TS a continuous data acquisition system has been built up which allows for the quasi real-time analysis of plasma data. Already for several seconds of pulse length in advanced regimes, active control of the plasma parameters is needed. All in-vessel PFCs reach a stable surface temperature after a few seconds. Not only plasma density and position are controlled, but also energy, temperature and current profiles. As profile sensors an IR-interferometer, a polarimeter, ECE heterodyne radiometers, and hard X-ray cameras are used. For the longer pulses feedback reaction of the heating systems LH, ICRH, and ECRH are included as well as particle control via pellets and gas valves and plasma position control via poloidal field coil currents [28-30]. Although otherwise quiet and apparently stationary, unexpected temperature oscillations are observed when approaching zero loop voltage after about $45 \mathrm{~s}$ in TS long pulse discharges as an interplay of the LH driven current density deposition profile and the local heat transport properties of the plasma [31]. It demonstrates the complex interplays, which are difficult to stabilize externally. Successful feedback experiments are reported also from other tokamaks operating in advanced regimes [26, 32-35].

High power long pulse operation demands for a careful detection and observation of transient processes like parasitic power loss, impurity production, or density rise as reported from the long pulse experiments in TS. Occurring only with modest amplitudes they might become dangerous under steady state conditions [36]. Long pulse discharges in TS can be limited by the production of supra-thermal electrons and ions in the $100 \mathrm{keV}$ energy range generated by LH and ICRH. They hit the wall onto very small spots where the power density limit of 10 $\mathrm{MW} / \mathrm{m}^{2}$ can be exceeded, rising the surface temperature to high values [37]. Hard X-ray tomography to diagnose runaway production and IR-monitoring are mandatory not only for safety reasons but also for diagnostic purposes to promote understanding. At TS an IR thermographic system has been designed to oversee the entire limiter surface and five RF antennae with a spatial resolution of $9 \mathrm{~mm}$ [5]. Calorimetry complements thermography by providing quantitative power loss measurements. Monitoring proper operation of the divertor including detachment, pumping and fuelling necessitates Langmuir-probes, pressure gauges, $\mathrm{H}_{\square}$-monitors, residual gas analyzers as well as erosion and dust monitors. Long pulse operation provides a unique opportunity to study particle balance and to address the problem of hydrogen retention in the wall, which does not reach saturation within minutes [38].

During long pulse operation of the superconducting helical system LHD, a large number of diagnostics are operated for both physics understanding, active feedback control and for interlock purposes. Maximum pulse length conducted so far is $3900 \mathrm{~s}$ [39] and the maximum total energy input through combined ICRH, ECRH, and NBI is 1.29 GJ [8]. There is no need to tailor and to keep the magnetic configuration since it is determined almost exclusively by the currents in the external superconducting coils. This makes plasma performance control far easier compared to tokamaks. Continuous operating diagnostics include FIR interferometers, 
ECE radiometers, visible spectroscopy of $\mathrm{H}_{\square}, \mathrm{C}$ II, C III, O II, O V, measurement of total radiated power, video observation of visible light and of various impurity lines. The FIR interferometer and the Rogowski-coils are used to actively control the line integrated density and the plasma current [40]. A spark caused by high-energy ions as generated by ICRH terminates the long pulse discharge. The spark results in a Fe X intensity increase connected with an increase of density and a sudden temperature decrease.

Early long pulse experiments with zero plasma current in a stellarator were conducted at the Advanced Toroidal Facility (ATF) with purely ECRH heated discharges [41, 42]. Pulse lengths of more than one hour (4667 s) were obtained with an input power of $70 \mathrm{~kW}$ from a $28 \mathrm{GHz}$ gyrotron with helium as the working gas. Thermal equilibrium of the wall was nearly obtained. Plasma control was achieved by feedback of the line density and the vessel pressure to keep the electron density below the cut-off density for second harmonic ECRH. The long pulses allowed for manual interaction setting the reference level of the density feedback. This mode of operation resulted in the longest sustained discharges. Hydrogen discharges could be conducted up to 20 minutes. Longer operation was not possible due to a loss of density control probably due to wall loading, demonstrating the importance of the plasma wall interface for steady state operation.

The long pulse discharges were conducted with only a small number of diagnostics, since all but two viewing ports were removed from the vessel after some were damaged during the long pulses by the microwave stray radiation (see chapter 6). In addition to the residual gas analyzer, a microwave interferometer, a diamagnetic loop, and an ECE radiometer were used.

\section{Diagnostics to permit device protection}

Protection of all in-vessel components of a fusion device is of importance, it becomes a seriuos issue for machines with pulse lengths in excess of $10 \mathrm{~s}$. Since typical thermal load at the $10 \mathrm{MW}$ heating power level ranges from several 10 to a few $100 \mathrm{~kW} / \mathrm{m}^{2}$, a typical diagnostic window of $100 \mathrm{~mm}$ in diameter is loaded with about $0.5 \mathrm{~kW}$, which can be handled by inertial cooling for a few seconds. However, going beyond, operation without active cooling of all in-vessel components is not possible at all. Thermal load varies as a function of toroidal and poloidal positions in particular in stellarators as shown in Figure 1 depending in addition on the magnetic configuration conducted. Cooling requirements for long pulse operation should therefore refer always to the maximum load expected. Stationary cooled PFCs are not only mandatory for safety reasons but also a necessary condition for density control. Careful observation of the surface temperature of all PFCs by IR thermography is necessary. It is of great importance in particular for all high heat load components like RF antennae and divertor target elements which might withstand $10 \mathrm{MW} / \mathrm{m}^{2}$ or even more, but could irreversibly be damaged when overloaded [43, 44]. Again IR-cameras for surface temperature monitoring play an important role. They are basically capable of providing the measurements with sufficient spatial and temporal resolution, but surface morphology might cause misinterpretations, which can be overcome in part by simultaneous measurement at different IR wavelengths [45-47]. The development of real-time algorithms for image processing to trigger safety feedback is another issue. Since absolute power balance is difficult on the basis of thermography, calorimetry of coolant's flow rate and temperature rise for as many as possible PFCs is reasonable for local quantitative measurements and to determine the global balance of injected and extracted power [45]. Almost 600 components are controlled in this way at TS. A similar number is foreseen for W7-X. 
Additional key parameters must be measured at a high level of reliability for machine protection: hard X-ray tomography for runaway detection, erosion monitors, halo currents, disruption precursors for disruption prevention in case of tokamaks, arcing monitors to control in particular wave launchers of ICRH, LH, and ECRH. Pulse-limiting arcing at or near to the antennas has been reported to occur still after a relatively long time of $85 \mathrm{~s}$ (TS) or after 1641 $\mathrm{s}$ (LHD) of otherwise quiet discharges. They are typically connected with a density increase and an increase of metallic impurities $[48,49]$. The increased radiation level results in a rapid decrease of the electron temperature. The abnormal termination observed in LHD is probably caused by local heating by high energy ions generated near the ICRH antenna system [49]. All diagnostic systems incorporated into the device protection monitoring must be built to operate with extreme reliability, unaffected by a possibly high level of steady state RF background radiation, and unaffected by erosion and coating of sensible PFC. Under BPX conditions, however, radiation effects will outperform and dominate all problems mentioned before.

\section{General considerations for long pulse diagnostics}

The selection of diagnostic systems dedicated for long pulse experiment is basically not different from the approach conducted to equip present day devices operating with pulse lengths below about 10 seconds. It is determined by the aims of the experiments, its scientific mission and the parameter ranges envisaged. It differs in the impact diagnostics need to have to keep plasma performance and to permit device protection, as discussed in chapters 3 and 4 . After defining the requirements for spatial and temporal resolution, diagnostic methods must be selected fulfilling the demands. Finally the diagnostic set can be assessed and assigned to the appropriate ports for optimum sightline conditions. Taking ITER as a model approach [50] this procedure for example has also been applied to establish the diagnostic system for W7-X necessary and sufficient to achieve its scientific mission and to verify the rigorous stellarator optimization realized for the first time with this machine [51-54]. Priority levels have been defined adapted to the various operational phases of the experiment. After that, feasibility studies were conducted concerning mechanical construction and necessary space to avoid collisions with other components in the vicinity of the machine.

Diagnostics for stellarators and helical systems are basically not different from those for tokamaks, however, they might have additional features like the possibility to measure the vacuum flux surfaces and they must be adapted to the intrinsically 3D structure of the plasma including in particular also the multi-dimensional divertor [55]. More emphasis compared to tokamaks might have to be put on the measurement of local electric fields because of their strong influence on stellarator transport in the fusion relevant low collisionality regime and in particular on the verification of the design elements of optimization since stellarators strongly rely on its successful implementation [53]. Amongst many others they include high-density diagnostics, sensitive current diagnostics able to measure the relatively small bootstrap and Pfirsch-Schlüter currents, diagnostics to measure the Shafranov-shift and those to prove fast particle confinement [51]. Tokamaks on the other hand may focus upon diagnostics to verify advanced scenarios including internal transport barriers, control of instabilities, and current drive control [35]. 


\subsection{Difficulties associated with long pulses}

Most of the diagnostic methods are able to operate steady state. However, to diagnose in an adequate way and along the proper sightlines, a number of sensitive components like detectors, preamplifiers, cables, retro-reflectors, lenses and mirrors are usually placed inside the ports or even inside the vacuum vessel relatively close to the plasma. All these components are vulnerable to thermal load and may suffer from erosion and deposition of wall material, which dramatically could affect their optical properties under long-pulse conditions. In addition, in particular in case of W7-X being equipped with $10 \mathrm{MW}$ of steady state compliant ECRH, all these components must be able to operate in an environment characterized by a high level of ECRH stray radiation. The associated problems will be discussed in chapter 6 .

The principal difference between implementing diagnostics in present day devices with extended pulse lengths and in BPX is the radiation dominated environment in which some of the key diagnostics will have to be installed and operated. The flux levels of the potentially damaging $\mathrm{n}$ - and $\mathrm{G}$-radiation, of photons and particles will be higher by orders of magnitudes compared to DT-operation in existing devices [56]. Therefore diagnostic selection has to cope with phenomena not previously important in diagnostics design. The most developed BPX diagnostic set has been prepared for ITER consisting of almost 50 individual measuring systems for machine protection, plasma control, and physics studies [3]. While still open questions exist $[2,14]$, the majority of diagnostic requirements assessed could be satisfied by adequate proposals based on existing experience or based upon special programs conducted to verify the applicability or to solve the problems associated with the harsh environmental conditions [57-59].

Main difficulties associated with long pulse operation in conventional devices are caused by loading of the diagnostics front-end by plasma radiation and particles either high energy atoms produced by charge exchange processes in the plasma or re-deposited sputtered material of the divertor and the first wall of the vacuum vessel. Thermal loading of PFCs by plasma radiation in the UV and VUV spectral regions can be of the order of $100 \mathrm{~kW} / \mathrm{m}^{2}$. It demands for active cooling of all PFCs, which can be quite difficult in case of optical windows as discussed in detail in chapter 9 of this paper. Re-deposition leads to coating on windows and mirrors by $\mathrm{Fe}-\mathrm{C}$ composite materials affecting transmission and prohibiting stable density calibration. All diagnostics based on optical observation of the plasma are affected. Unsolved issues here are the development of reliable actively cooled shutters with mechanical activation very close to the plasma to protect windows and mirrors in case they are not necessarily used, and the development of reliable cleaning techniques to remove the coatings. In case this is not feasible, methods for in-situ calibration need to be established. Generally, the construction of PFCs affected should be done in a way allowing for straightforward and uncomplicated maintenance or replacement.

As mentioned before, the other severe problem is connected with magnetic diagnostics. In long pulse discharges flux changes may for most of the time be very small. To obtain the quantity of interest, electronic integration of the voltage induced in the various loops has to be accomplished. Since all electronic circuits suffering drifts and noise, long time integration with existing integrators leads to unacceptable errors. Recent developments, however, seem to fulfil the demands and will allow for integration times into the $1000 \mathrm{~s}$ range. In addition, 
completely different approaches on the basis of the Faraday effect in fibre optics or rotating coils are being developed to fulfil the demands, as will be discussed in chapter 12.3 and 12.4.

Stepping forward to BPX devices, for example to ITER, neutron flux at the first wall of up to $10^{18} / \mathrm{m}^{2} \mathrm{~s}$, being still of the order of $10^{16} / \mathrm{m}^{2} \mathrm{~s}$ at the first mirrors in the diagnostic blocks will dominate all other interactions. It results in a volumetric heating of almost 10 $\mathrm{MW} / \mathrm{m}^{3}$ accompanied by $\square$ radiation at a dose rate of $10^{3}$ and $10 \mathrm{~Gy} / \mathrm{s}$ respectively, causing radio luminescence and modifications of in-vessel dielectric material [10]. CX-particle bombardment with fluxes of $10^{17}$ to $10^{18}$ per $\mathrm{m}^{2} \mathrm{~s}$ cause surface modifications of plasma facing mirrors degrading their optical properties [60,61]. As mentioned in the introduction, the wide field of these radiation-induced effects will not be covered here. Information can be found in the references mentioned before [10-13, 62, 63].

\subsection{Opportunities associated with long pulses}

Long pulse operation may result in the longer term in completely different modes of operation of a fusion device with real-time analysis of most of the diagnostic data, with extensive feedback control directly acting on the heating and current drive systems for multidimensional parameter scans or scans of diagnostics parameters. Even if this is still far ahead of today's experimental scenarios, long pulse steady state discharges have been conducted providing a number of diagnostic opportunities [64]: At LHD a $3 \mathrm{~m}$ VUV spectrometer has been slowly rotated vertically $(0.02 \mathrm{deg} / \mathrm{s})$ to measure the spatial profile of the spectrum [40] as well as toroidally providing 2-dimensional spectra [65]. In another example a polarizer in front of the ECE spectrometers has been rotated to determine the polarization state of the cyclotron emission [66]. Scanning of the neutral particle analyzers (NPA) provides a measure of the loss cone [67] or can provide a full radial profile of the ion energy with high spatial resolution. Many other diagnostics suffering sufficient signal-to-noise ratio can benefit from long pulse steady state discharges by time averaging of the signals. Examples are TVThomson scattering, X-ray CCD cameras as well as imaging bolometry [68, 69]. A new possibility could arise by applying modulated cw-laser Thomson scattering. For example a modest $(10 \mathrm{~W})$ modulated argon ion laser could deliver sufficient photons $(5 \mathrm{~J}, 50 \%$ duty cycle) in $1 \mathrm{~s}$. The modulation would enable clean discrimination against background. Longer integration times would allow for the use of phototubes and phototube arrays.

Many kind of correlation studies in the frame of fluctuation measurements need long observation times to reach the significance level like core temperature fluctuation measurements or active modulation experiments for transient transport studies relying on coherent averaging [70-72]. They all will benefit from long pulse stationary conditions.

\subsection{Data acquisition}

A major challenge in long pulse experiments is the data acquisition system. Present short pulse experiments evaluate the shot data after the discharge. Data collection typically takes a few minutes until diagnostic data are available for the data analysis software modules. In long pulse experiments, however, data evaluation must be possible during the discharge as well as for monitoring purposes or even for active plasma control on longer time scales. A precondition for satisfying the demands is a well structured storage of huge amounts of data. Typically, this is done by means of a database, which could be a particular development for fusion experiments needs like the MDSplus [73], which is used at many fusion experiments but is not steady state capable, or commercially available ones, preferably object databases 
due to the high performance needs and the complexity of the data structures. The data structure itself may change in time, for instance if diagnostics are activated or deactivated during the discharge.

A further challenge is the data acquisition at high sample rates, as needed for fluctuation diagnostics, and the data acquisition of camera data. Taking a typical diagnostic set into account, the data streaming capabilities of present hardware and software architectures of the data acquisition station and the data transfer rate and random access times of databases are likely to be insufficient. However, LHD has reported on a steady state capable data acquisition system [74], which allows the integration of existing non steady state capable CAMAC systems. This system is based on object-oriented databases and a compact PCI bus system for the fast digitizers on the DAQ station side and additionally on a Gigabit network technology. A continuously running data acquisition with $84 \mathrm{MB} / \mathrm{s}$ transfer rate to the host main memory could be demonstrated. A similar data acquisition system is reported for HT-7U [75]. A new data acquisition system is under development for W7-X employing a different structure dedicated for steady state operation [76]. In this system, the DAQ station has demonstrated streaming rate to the archive of $7.8 \mathrm{MB} / \mathrm{s}$.

In case of insufficient streaming data rates, several methods for data reduction exist. The first one is to define time windows, in which diagnostics signals are recorded at higher sample/frame rate and higher resolution. Such a system requires ring buffers inside the DAQ station and dedicated precise hardware for employing post-trigger logics [77]. The fast DAQ windows can be predefined in the central discharge control system, but also triggered by events in the plasma. This approach is driven by physical interest on long discharges. The physics studies motivated investigations are typically done on shorter time intervals given by the longest time constants of the plasma itself, predominantly the $L / R$ and current diffusion times. Thus, the physically motivated phase lasts approximately one or two minutes. Longer discharges are determined mainly by some technical aspects, i.e. thermal loading of first wall elements, particle recycling and the evolution of impurity concentration in the plasma. A long discharge could be recorded, thus, with a reduced diagnostic set, reduced sample rate and without or at least a strongly reduced fluctuation diagnostic set. However, taking into account the cost of a discharge in a superconducting device and the demand of analysing the unexpected events, this kind of data reduction should be avoided whenever possible. The second method is the data reduction by compression algorithms either with or without information loss. For imaging data like video diagnostics, even well developed algorithms as used for multimedia applications in consumer electronics may be used. However, in case of any machine operation failure all details in video images are of interest and in case of other camera diagnostics quantitative analyses do not allow any loss of information when archiving camera data [78]. In particular modern video reduction algorithms show artefacts at sharp edges and when the brightness of the image changes suddenly.

The general trends in data acquisition systems, the historical development and steady state aspects have been summarized in [79].

\section{Non-absorbed ECRH stray radiation}

In stellarators plasma start-up can favourably be achieved by RF-breakdown with ECRH. This is the typical scenario conducted at the former W7-AS device, the same is planned for the future W7-X. It will be equipped with 10 steady state gyrotrons operating at $140 \mathrm{GHz}$ with a 
maximum total output power of almost $10 \mathrm{MW}$ launched into the machine [80]. Under these conditions the danger of damage by non-absorbed ECRH radiation arises. Direct irradiation of non-absorbed beam power may cause local heat load. To avoid damage and hot spots, components opposite the beam launchers must be armoured and actively cooled. The measure is especially important if heating scenarios will be conducted with low first pass absorption of the primary beam. Non-absorbed microwave power will partly be absorbed at the inner wall, but the major part will be reflected building up, after multiple reflections in the vacuum vessel, an almost isotropic radiation level endangering a variety of in-vessel diagnostic components.

This background radiation level does not only affect built-in sensitive microwave diagnostic components but is high enough to thermally load windows, valves their sealing etc. Test measurements at W7-AS and FTU as well as code calculations have been conducted to simulate the expected radiation level and its distribution around the torus [81-84]. The level depends on the plasma parameters and the heating scenario conducted: the mode the primary beam is launched, ordinary $(\mathrm{O})$ or extraordinary $(\mathrm{X})$ mode, 1 st or 2nd harmonic of cyclotron frequency, or under conditions for current drive or for mode conversion to Bernstein-waves as conducted at high densities [85-87].

\subsection{Expected stray radiation level}

The power flux density of stray radiation expected inside the vessel is determined by the total input power, $P_{i n}$, the total power absorbed in multiple passes through the plasma, $P_{a b s}^{\text {(plasma })}$, and multiple reflections at the wall $P_{a b s}^{(\text {wall })}$, as well as the amount of power escaping through the area $A_{F}$ into ports and through the windows, $P_{l}^{\text {(window) }}$

$$
P_{i n}=P_{a b s}^{(\text {plasma })}+P_{a b s}^{(\text {wall })}+P_{l}^{(\text {window })} .
$$

The power flux density $p$ of stray radiation can usefully be estimated considering the single pass absorption $\square_{1}$, the wall absorption $\square_{W}$, the total wall area $A_{W}$, and the average plasma absorption $\left\langle\square_{P}\right\rangle$ in the plasma resonance layer area $A_{P}$ in multiple passes through the plasma following the wall reflections,

$$
P_{\text {in }}\left(1 \square \square_{1}\right)=p\left[\square_{W} A_{W}+A_{F}+\left\langle\square_{P}\right\rangle A_{P}\right]
$$

Considering the wall and window areas of $\mathrm{W} 7-\mathrm{X}$ results in up to $100 \mathrm{~kW} / \mathrm{m}^{2}$ power flux density under conditions of $60 \%$ first pass absorption in the plasma and $5 \%$ wall absorption during each reflection. This situation is typical for current drive or mode conversion scenarios, however, comparable low first pass absorption is expected as well at electron densities close to the cut-off densities of X2- and O2-modes.

The estimates simply based on a power balance assume homogeneous power distribution in the vessel. Geometrical details of the vessel are not included. To calculate the power distribution with higher accuracy, a model of coupled cells has been used [83] and 3dimensional ray-tracing calculations conducted $[81,84]$. The different codes have been tested by checking against measurements of non-absorbed stray radiation in W7-AS and FTU. [88]. Good agreement has been obtained. Subsequent calculations for W7-X show that the power flux density decreases by about an order of magnitude with increasing distance to the ECRH launching poloidal plane of the torus. Both the absolute value at any position inside the vessel 
and the rate of attenuation with distance depend on the wall absorption. Wall coating with boron carbide $\left(\mathrm{B}_{4} \mathrm{C}\right)$ results in about $30 \%$ absorption during beam reflection compared to only about $5 \%$ of graphite. $\mathrm{B}_{4} \mathrm{C}$ coating of all in-vessel surfaces could reduce the stray radiation level to almost negligible values. Thermal loading of in-vessel components owing to non-absorbed ECRH reaches under certain conditions the level of plasma radiation or might even exceed it. It must carefully be taken into account considering cooling of in-vessel components in long pulse experiments. In case of VUV, XUV plasma radiation only components directly facing the plasma are affected. Microwave radiation, however, propagates as well behind divertor components and into gate valves and might build-up high electric field strengths in these areas.

\subsection{Specific effects}

Specific interaction with the $140 \mathrm{GHz}$-radiation is expected only in case of microwave diagnostics operating in or near to this range. These are basically all microwave diagnostics, ECE, reflectometry, polarimetry, and interferometry. Although extremely sensitive detectors are used in these systems directly responding to the wave, counter-measures are possible and relatively straightforward to establish since narrow-band microwave notch-filters with an attenuation of several orders of magnitude are available which can be integrated into the signal path to reduce the radiation level to acceptable values.

\subsection{Non-specific effects}

Unspecific thermal effects based on dielectric losses are more difficult to avoid. Among the diagnostic systems, which might strongly be affected are the soft X-ray camera arrays in particular the detector diodes and the thin metal foil filters in front of them. In VUV spectroscopy the channeltron detectors could be damaged, in the UV region the vacuum windows, the fibre optics, and the CCD cameras used are exposed to danger, and especially the bolometer cameras must carefully be protected by high pass filters in front of each absorber element. Even heating the probe-tip insulating material of Langmuir-probes need to be taken into account. Heating of a zinc-selenide IR window by non-absorbed ECRH at 53.6 $\mathrm{GHz}$ in TJII produces an optical path length difference between the two laser beams of the two-colour interferometer $\left(\mathrm{CO}_{2}, \mathrm{He}: \mathrm{Ne}\right)$ introducing significant errors in the line density measurements [89].

All windows and in particular the sealing of the vacuum windows as well as the sealing of the vacuum gate valves must carefully be investigated concerning their absorption properties. Low loss materials for the $\mathrm{mm}$ - and sub-mm wavelength regions are listed in $[90,91]$ and the references given therein. Dielectric loss is usually expressed by the so-called loss tangent, $\tan \square=\tan (\sqcap / \square)$, of the complex dielectric constant $\Pi=\Pi \Pi i \Pi$. The loss tangent of materials used for vulnerable diagnostic components inside the torus should stay below about $10^{\square}$ (see chapter 9 for more details).

Large differences in the absorption of O-rings of valves have been measured at $140 \mathrm{GHz}$ ranging through many orders of magnitude for various materials. Pieces of $10 \mathrm{~mm}$ length and $4 \mathrm{~mm}$ diameter have been measured in a standard test set-up. The most significant results of three trade products of sealing materials are listed in Table 1: 
Table 1

\begin{tabular}{|l|l|}
\hline Material & Attenuation $(\mathrm{dB})$ \\
\hline Viton & 22 \\
\hline Kalrez 1050LF5 & 11 \\
\hline Kalrez 40017 & 1.5 \\
\hline
\end{tabular}

Early long pulse experiments under steady state ECRH conditions conducted at ATF revealed the whole problem [41]. To prevent damage to the Viton O-rings in the gate valves to the pumps, a microwave cut-off screen had to be installed. Other gate valves were removed from the machine. Viton rings were exchanged by silicon rubber rings, which proved to have a higher resistance to the microwave radiation. Also many viewing ports were removed from the vessel, since they were damaged during the long pulses. Microwave screens were installed in front of the diagnostic windows

Following this experience a large stray radiation test chamber fed by a $140 \mathrm{GHz}$ gyrotron has been built to test all in-vessel, and in particular the in-vessel diagnostic components for W7-X $[92,93]$. The cylindrical vacuum chamber made of aluminium of $2 \mathrm{~m}$ in length has a total volume of almost $4 \mathrm{~m}^{3}$. Due to the high reflectance of the aluminium wall material, the quality Q-factor of the chamber is measured to be of the order of $10^{6}$. This quantity determines the power flux density finally obtained for a given input power. The gyrotron feeding the chamber can be operated in pulsed mode at about $3 \mathrm{~Hz}$ without time restriction when running with a duty cycle of about 10 . The output power in the pulse is $180 \mathrm{~kW}$. This results in a measured average power flux density of homogeneous isotropic radiation of almost $30 \mathrm{~kW} / \mathrm{m}^{2}$, comparable to the values expected during operation of $\mathrm{W} 7-\mathrm{X}$. Homogeneity and isotropy within the chamber have been reached by applying a dedicated microwave launching scheme with the high power beam irradiating at grazing incidence to the wall [94] as shown in Figure 2. The devices to be tested are put into the central part of the chamber where homogeneity and isotropy of the power flux density are maximum to get most reproducible results. Test campaigns will be conducted to investigate the behaviour of all components to be mounted inside the W7-X vacuum vessel. The chamber is equipped with adequate diagnostics, thermocouples, an infrared camera, multi-mode so-called sniffer probe detectors [88] and mass spectrometry to investigate the behaviour and proper functioning of diagnostic components under the conditions of high background microwave radiation.

\section{Problems and considerations for standard diagnostics}

Specific problems connected to steady state operation of the most important and most affected diagnostics are briefly reviewed. Again we focus more on long pulse operation rather than on operation at BPX conditions within a highly radioactive environment. As mentioned before, specific BPX measurement requirements and dedicated BPX diagnostic solutions are not reviewed here at all. Diagnostic problems under long pulse conditions are mainly connected with the very front-end of most important optical diagnostics. They will be reviewed in chapter 9 .

Some problems not mentioned so far might be general to all diagnostic systems: 
(i) Long pulse operation results in temperature excursions of the vacuum vessel giving rise to thermal expansion and relative movements which will be up to about $10 \mathrm{~mm}$ both in radial and vertical directions in $\mathrm{W} 7-\mathrm{X}$ and up to several $\mathrm{cm}$ in ITER which must finally be accommodated in particular by the optical components of the diagnostic systems [95, 96].

(ii) Since accessibility to the torus hall of long pulse experiments with superconducting magnet systems is restricted when magnets are energized, all systems inside the bio-shield and inside the vessel need maintenance by remote handling, complicating their design and cost to a great extend, a fact of extreme importance for BPX. Under these conditions also realignment and re-calibration procedures become at least time consuming.

The necessarily high reliability requested demands for redundancy and the development of novel robust measuring techniques. One aspect, already mentioned in chapter 2, is important in this context. It might become indispensable in case of automated real time data analysis during long pulse operation, that is integrated data analysis (IDA). Traditionally diagnostics are designed as individual independent systems, however, substantial benefits can be obtained from an integrated approach where complementary diagnostics are synergistically combined to form a single measurement system $[20,97,98]$. Such a system should significantly improve overall reliability and accuracy. All diagnostics able to deliver information on one plasma parameter, the electron density for example, that is interferometry, Thomson scattering, and bremsstrahlung emission measurement, must be designed from the very beginning as one common system. Their sightline orientation, the parameter range covered etc, should be selected for maximum information and smallest error of a combined analysis [99].

\subsection{Magnetics}

Magnetic probes are amongst the important diagnostics measuring magnetic field changes caused by changes of the current inside the plasma. Different aspects of the plasma current distribution such as the total current, the diamagnetic current, or the dipole field of the parallel currents are of interest. In stellarators, in particular W7-X, all currents are very small. However, an accurate measurement of bootstrap and Pfirsch-Schlüter currents is mandatory to verify the neo-classical optimization applied $[51,53]$. The standard way to perform the measurement is to use coils or loops outside the plasma and to integrate the induced voltage to obtain the magnetic flux through the coils. Various types of coil arrangements like Rogowskicoils, diamagnetic loops, saddle coils, flux loops, and poloidal field coils represent the standard instrumentation [100]. The whole set will be used for equilibrium analysis as well and provides information on plasma shape and position and gives information on halo currents in supporting structures.

The main problem of magnetic measurements arises from offset drifts of the electronic integrators used. Standard integrators can be used for integration times of up to about $100 \mathrm{~s}$, meaning that a reduction of the offset drift by about one order of magnitude is necessary for steady state operation as envisaged. The problems are discussed in detail in chapters 12 of this review.

Besides thermal problems with cooling of the coil housings integrated into the first wall, nand $\square$-radiation induced effects as unavoidable under BPX conditions are the most serious challenge. Different interactions have to be distinguished affecting in particular the ceramic insulation of the coil wire. The breakdown voltage of the insulator and its conductivity are unfavourably influenced, and additional currents and voltages appear in the coils as a whole basically indistinguishable from true signals. More information on this subject is summarised in chapter 11. 


\subsection{Probes}

Probes are traditionally the standard diagnostic to probe the particle distribution in the very edge of the plasma. Information on the local electron temperature, the ion saturation current and the plasma potential can be derived from the current-voltage characteristic of the probe [101]. During long pulse operation probes have to be actively cooled in order to avoid thermal overloads. Adequate solutions are, however, technically very complicated. Moreover, targetintegrated probes at fixed positions have to be flush-mounted which involves problems concerning the interpretation of measured probe characteristics. To avoid active cooling only movable probes are being considered. Mounted on a sufficiently fast manipulator $(>1 \mathrm{~m} / \mathrm{s})$ or pneumatically driven target integrated pop-up arrays allow for the short exposition time of $100 \mathrm{~ms}$ in maximum [102, 103]. Most critical elements are the ceramic insulators enclosing the carbon probe tip in order to provide well-defined probe surfaces. When exposed to the plasma, the convective thermal load together with plasma radiation could exceed the maximum tolerable load of about $1 \mathrm{~W}$ for such components. While current loading occurs only when in contact with the plasma and thermal radiation is tolerable, radiation during BPX might strongly affect the material properties of the insulator used $[10,11,13]$.

\subsection{Spectroscopy}

Spectroscopic diagnostics cover a wide spectral range from X-rays to visible with both passive and active techniques applied to diagnose the plasma core, the radiation mantle, the scrape off layer, as well as the divertor plasma in various ways. Most important parameters as impurity species identification and their concentration, the ion density and temperature, the electron temperature, the effective ion charge, the plasma rotation, impurity transport, internal magnetic fields and many others are routinely deduced from measurements of line intensities, line width, Doppler-shift, and bremsstrahlung continuum intensity [104]. Basically grating and crystal spectrometers and the various types of detectors ranging from CCD-cameras to channel plate detectors and photomultipliers are long pulse compatible. Gain control and baseline drifts are not reported to be a problem.

Spectroscopy in the visible has technical advantages because of its relative ease to combine a large number of sightlines guided via optical fibres to spectrometers outside the torus hall however, the fibres might strongly be affected under BPX conditions and cannot be used anymore (see chapter 10 for more details).

The long-term stability and quality of the very front-end of the optical components close to the plasma boundary is a most critical issue. Intensive studies on coating effects and the development of active schemes including cooled shutters and on-line calibration techniques are currently undertaken and explored [61, 105]. The main problem connected with spectroscopic diagnostics is the stability of the optical transmission of the vacuum windows involved. In many cases due to aperture reasons they must be positioned very close to the plasma resulting in coating of the windows during plasma operation. Telescope arrangements need to be developed able to pass coated windows through locks for cleaning purposes. In addition shutters right in front the windows must be installed. Possibly most of the time during long pulse operation they will be closed and opened only for short times in given intervals. More details are given in chapter 9 of this paper.

Spectrometers in the VUV $(10-150 \mathrm{~nm})$ and XUV $(1-10 \mathrm{~nm})$ spectral ranges must directly be coupled to the machine vacuum vessel. The optical transmission path does not allow for any blocking by windows or transmission filters. Interference and heating problems connected 
with ECRH stray radiation may arise. Screening grids introduced into the optical path reflecting the mm-wave radiation could be a solution.

It appears that virtually standard X-ray diagnostics can be adapted if careful shielding of detectors in particular in BPX is achieved. Compact solid-state detectors as well as proportional counters are most sensitive to the high radiation background of neutrons. For example, crystals and detectors are arranged in Johann-type spectrometers in such a way that the detectors are not directly affected by n-radiation from the plasma. Well-collimated beams make sufficient shielding of the detectors possible [106]. Basically additional reflections in mirror-relay-arrangements must be considered to deflect the X-ray beam out of the neutron path. The measure has the additional purpose to reduce n- and $\square$-induced noise generating a high count-rate pedestal necessary to be subtracted and causing additional statistical shot noise errors.

Active CXRS measurement relying on a dedicated diagnostic neutral beam (DNB) as considered for $\mathrm{W} 7-\mathrm{X}$ and for ITER will provide information on low-Z impurities and by measuring the line profile also local information on the ion temperature is gained. In BPX it might also be the sole approach to measure the He-ash [107]. No specific long pulse problems seem to be connected with active measurements. Since the DNB will be a pulsed system, spectroscopic systems can be operated in pulsed mode as well with the dedicated first mirrors protected by shutters during the DNB off-phases. Additional open phases are only necessary to determine the plasma-only component of the spectral emission. The DNB can also be used in tokamaks for measurements of the motional Stark-effect (MSE) of Balmer- $\square$ components to derive the local current density from the poloidal to toroidal field pitch angle. Since the polarization states of the Balmer- $\square$ line components need to be determined in MSE measurements with high accuracy, the polarization changes of the front-end mirrors due to surface coatings will be of particular concern [108]. In spite of polarization distortion, it could still be possible to estimate $\bmod (\mathrm{B})$ based on the splitting of the Stark-multiplet. In addition, using appropriate polarization modulation and spectral discrimination techniques, it should also be possible to measure at least the temporal evolution of the orientation of the induced electric field (though not its absolute orientation) even in the presence of window- induced elliptization.

\subsection{Thermography}

Application of IR-thermography is used to conduct the total power balance of injected and extracted power and in particular in long pulse experiments to survey all power loaded invessel components [45, 46, 109]. Another application is connected with the successful operation of the divertor relying on semi-attached plasmas controlled amongst others also via thermographic monitoring. Fast algorithms have been developed and applied to IR thermocamera data to build-up interlocks reacting within about $0.2 \mathrm{~s}$ for the reliable protection of the sensitive targets elements. Only thermography can provide the accuracy, the dynamic range, and sufficient temporal and spatial resolution to detect most dangerous hot spots. Thermography is usually complemented by numerous thermocouples in a calorimetric diagnostic system forming the necessary control tool for absolute calibration and for power balance calculations, however, without having sufficient temporal and spatial resolution demanded for fast safety purposes.

As with all optical diagnostics, the quality of the PFOCs is essential for reliable operation. Depositions on first mirrors might be detected by in-situ calibration via carbon shutters or other build-in blackbody sources [45]. Additional problems arise from surface morphology effects, dust and co-deposited semi-transparent layers influencing the temperature measurements through local emissivity variations at given wavelength. 
In TS inspection of the inner vessel wall revealed the presence of carbon deposits of carbon evidenced by IR imaging appearing as hotter zones as the thermal conductivity is reduced vertically and making temperature measurements of PFC components very difficult [47]. Surface roughness is influencing in a similar way. The problems can partly be overcome by monitoring simultaneously at different wavelengths [110].

\subsection{Bolometry}

The determination of the total radiated power is of importance for the total power balance as well as to verify quantitative spectroscopy. Multi-sightline tomographic reconstruction using several hundreds of lines gives the spatial distribution of the radiated power important also for basic and advanced plasma control in feedback loops with gas puff fuelling [38]. In the standard detection system one makes use of the temperature sensitive resistance of gold meanders mounted on mica substrates, arranged to form a pinhole camera. The power absorbed is deduced from the differentiated temperature rise of the absorber elements. Electrical bridge circuits are used for resistance measurements. They are suffering from uncompensated thermal signal drifts difficult to correct in case of long pulse experiments. Active cooling must be provided to keep the bolometer heat sink at constant temperature to high accuracy. Another drift problem arises due to small differences in the resistors forming the electrical bridge. Simultaneous measurement of the bridge currents could improve evaluations [111]. One possible solution might be using two bolometer systems simultaneously with shutters alternatively closed to determine the baseline of the amplifiers during off-cycles. The systems are in addition sensitive to $1 / \mathrm{f}$ and shot noise. Using an ACbridge arrangement at sufficiently high chopper frequency, the $1 / \mathrm{f}$-component can be avoided. Additional problems arise from pick-up in the long leads from the sensitive front-end detectors mounted near the plasma to the subsequent amplifiers typically some distance away [112].

Another type of bolometer system views the temperature rise on a thin metal foil with an IR imaging camera. An early version of this infrared imaging bolometer relied on segmented copper masks to divide the bolometer foil into thermally isolated channels [113]. In a later concept known as the imaging video bolometer (IRVB), a single large gold foil is used and the two-dimensional heat diffusion equation in the foil is solved for the incident radiated power density [114]. In these diagnostics hundreds to thousands of channels without wire leads to the individual foil elements are realised, however, so far connected with a reduction of sensitivity by about a factor of 2-20 compared to metal foil detectors $[69,113,115]$. Since no wire connection is needed and no DC-amplifiers involved, the system is immune against magnetic pick-up and baseline drift and therefore more compatible with steady state operation. Prototype IRVB systems are being tested at LHD and JT-60U [114, 116].

For BPX applications the detector must be radiation hard to survive the $\mathrm{n}$ - and $\square$-fluxes. In case of the standard metal resistor absorbers this can be accomplished by changing from Au to $\mathrm{Pt}$ to avoid irradiation-induced damage by transmutation of $\mathrm{Au}$ to $\mathrm{Hg}$ and to replace the mica substrate by radiation hard ceramics with the platinum resistance tracks applied [117]. A $\square$ radiation hard bolometer development has been conducted at JT-60. The system made of gold absorbers on a polyamide sheet in a stainless steel mounting frame survived 220 MGy of fluence without significant change . 


\subsection{Thomson scattering}

Determination of the spectrum and the absolute intensity of laser light incoherently scattered from plasma electrons is amongst the standard methods to measure the local electron temperature and density [118, 119]. High power lasers with repetition rates up to $100 \mathrm{~Hz}$ are used. The classical scattering set-up uses perpendicular lines of sight for the laser input beam and the detection system. Different spatial points are measured with individual polychromators and detectors. The need for access to several ports can be overcome by applying backscattering arrangements so-called LIDAR systems whose key advantage is that only one access route is required. All spatial channels are viewed through one optical line, resulting in simpler optics compared to the wide field of view collection optics necessary in a conventional system. In addition automatic alignement is obtained between the primary laser beam and the detection system. Important for calibration is the fact that different spatial points are measured by the same set of detectors. Localization is accomplished by a time-of-flight measurement [120].

The relative spectral sensitivity of the detection system which must be known to infer the electron temperature from the scattering signals is measured by illuminating with a calibrated, tuneable, monochromatic light source or using tungsten continuum sources. This source is usually placed outside the vessel thus the transmission of the windows and the optical invessel components is not measured after the start-up of the plasma experiments. Absolute calibrations of the density measurements of the standard Thomson-scattering system are conducted applying Rayleigh- or Raman-scattering by filling the torus with a molecular gas. Cross-calibrations are possible against reflectometer and interferometer/polarimeter results. In LIDAR systems relative measurements are possible without calibration since the variation of the detection solid angle is determined exclusively by the observation geometry. It can be calculated or measured before start-up of a machine. It does not vary with time.

Main concern during long pulse operation is the stability of the PFOC components. Coating of first mirrors and windows will affect in particular the density measurements, however, since the scattering spectrum is rather wide extending over the whole visible spectral range, dispersion due to coating might affect severely also the temperature evaluation [121]. Tests are conducted to develop a method of re-calibration by measuring the scattering distribution at different laser wavelengths on the same plasma within a short time interval [122]. Problems with the optical components are not only due to interaction with the plasma. The high intensity of the primary laser beam might destroy windows and mirrors as well [123]. A water-cooled beam dump is mandatory.

Additional problems may arise from the stability of the laser pulses. Nd:YAG lasers are typically used with high repetition rates, able to operate over $1000 \mathrm{~s}$ without significant degradation. However, laser power must simultaneously be monitored. On the detector side the polychromators will use interference filters and avalanche detectors. The detector electronics must be well stabilized to avoid temperature drifts during long pulses.

In BPX the survivability of the first mirror for spectral range 400-800 $\mathrm{nm}$, low sputtering yield and high laser damage threshold is again the main challenge. It has to maintain good optical quality in the presence of nuclear heating, plasma radiation and neutral particle bombardment. Rhodium coated mirrors with copper substrate are considered a possible candidate [60].

Coherent Thomson scattering (CTS) do determine ion features of the plasma is far away from being a consolidated diagnostic. The availability of long pulse high power gyrotrons, however, seems to open the way [124-127]. There is no general concern prohibiting steady 
state operation. Detection systems are comparable to the ECE systems described in the next chapter.

\subsection{Microwave diagnostics}

Microwave diagnostics ECE, reflectometry, interferometry, and polarimetry are briefly discussed in common. Although interferometry/polarimetry systems are often operated in the FIR or IR spectral regions, they are included since they historically evolved from the same physics basis. Standard microwave diagnostics probe the electron component of the plasma. For B-fields of several Tesla of typical magnetized fusion plasmas, cut-offs and resonances governing the dispersion relations are in the $\mathrm{mm}$ - and sub-mm wave ranges.

Electron cyclotron emission (ECE) at the blackbody level covering typically about 100 to 300 $\mathrm{GHz}$ of frequency is measured with high spectral resolution with absolutely calibrated scanning Michelson-interferometers and total power multi-channel radiometers [128-131]. Collection optics include aperture antennas either directly viewing the plasma or via mirror relay arrangements based on Gaussian optics $[132,133]$. Signal transmission to the detectors typically far away from the torus is conducted with low-loss oversized waveguides. Since the requirements concerning surface quality are at mm-wavelengths reduced by about 4 orders of magnitude compared to optical spectroscopy, no severe mirror problems are expected in long pulse experiments, although absolute calibration is requested. Actively cooled mirrors and antennas made of stainless steel or carbon-based materials can be used. Absolute in-situ calibration is accomplished before start-up and re-calibration is possible with in- or ex-vessel blackbody sources with shutters and return paths included into the transmission lines or even using the heated empty vacuum vessel itself [130]. Baseline drifts of individual radiometer video amplifier channels can be detected during long pulse discharges by switching the radiometer onto matched loads of known temperature for short intervals.

BPX conditions demand for labyrinths in the front-end optics for radiation shielding, however, no severe radiation damage problems are being expected with the first mirror. Interpretation problems may arise with ECE measurements since relativistic effects play a major role at the high electron temperatures of BPX. In ITER harmonic overlap will limit the range of major radius that can be investigated, cut-offs will limit the applicability of the method at high densities, and shine through will affect measurements at the plasma edge [128, $134]$.

Reflectometry is probing the plasma density profile with a kind of radar technique determining the round-trip time delay as a function of frequency of a wave sent to the plasma and reflected back from the cut-off layer. The profile can be determined after Abel-inversion [131, 135140]. Probing frequencies are typically in the range 15-100 GHz. Separate antennas are used for launching and reception of the wave. Flat parts of the density profile are accessible launching waves in X-mode polarization. As with ECE measurements, no severe problems are expected in steady state experiments. Antennas used for ECE and reflectometry as well as surface quality requirements are basically similar. The method only needs minimal access to the torus for the antennas. As with ECE all in-vessel components are robust. The sensitive elements, microwave sources and detectors are outside the radiation area. Cooling of PFCs under long pulse conditions is necessary. For calibration the distance to a well-defined reference plane is determined. In-vessel back wall mirrors could basically be used, however, if thermal expansions are to be expected in long pulse experiments, complete additional reference waveguide runs available via waveguide switches must be considered. 
Interferometry determines the line integrated electron density by measuring the phase delay of a wave propagating through the plasma compared to a reference wave outside. Multi-channel interferometers in parallel beam arrangements allow for the determination of the local density applying Abel-inversion [141-144]. The optimum wavelength is defined by a compromise between ray refraction and the sensitivity to mechanical vibrations of the optical components. Most experiments use wavelength in the FIR $(118 \square \mathrm{m})$ and rigid frames to get sufficient mechanical stability. If refraction is most important as with probing the steep density gradients in the plasma edge region, a wavelength in the IR is preferable (10.7 $\square \mathrm{m})$ [145]. A short wavelength makes refraction negligible but the resulting moderate phase shift requires highly sensitive phase measurements. The total round trip phase will only be comparable often much smaller than the phase changes from mechanical vibrations. Vibration compensation in a two colour scheme is mandatory [146-148]. Generating the second wavelength via harmonic generation by doubling the frequency is advantageous in case optical path length changes by the plasma are smaller than those introduced by mechanical vibrations.

If separate ports for beam input and exit are not available, retro-reflectors must be mounted inside the vacuum vessel. In this case coating might give rise to problems in particular if the second wavelength in the two-colour scheme used is in the visible. Two wavelengths in the IR are then preferable on the expense of larger errors in the vibration compensation. During long pulse operation spatial drifts of the laser beams away from the most sensitive area of the detector diodes due to thermal deformations of optical components and drifts in mirror adjustments must be avoided by feedback control applying four-quadrant detectors and stepper motor driven mirror alignment [149].

Long pulse operation demands for a robust measurement of the line-integrated density for control purposes. Since interferometers suffer fringe jumps, which cannot be avoided completely, polarimeters are developed for this task [142, 150,151]. Since no fringe counting is necessary, even interruptions causing total signal loss are tolerated. Both circular and linear bi-refringence of magnetised plasmas is exploited in Faraday- and Cotton-Mouton-effect polarimeters (ellipsometry). Both effects appear basically simultaneously depending on the sightline chosen [150, 152]. Proof-of-principle experiments under the unique B-field conditions of a stellarator have successfully been conducted at W7-AS with a single channel Cotton-Mouton polarimeter operated at about $1 \mathrm{THz}[153,154]$. Similar systems are proposed for ITER as well as a tangential Faraday-polarimeter with multi-sightlines in toroidal direction operated in the FIR [155]. Since the vacuum field is known, the quantity of interest can be unfolded from the measurement.

Faraday polarimeters, also combined with an interferometer as interfero-polarimeters [156], with sightlines oriented in a poloidal plane are measuring the Faraday-rotation of a linear polarized wave due to the parallel B-field component from which in tokamaks the q-profile and the current density distribution can be deduced [157]. Long pulse operation seems not a problem, quite the contrary. Small Faraday rotation angles can advantageously be determined making use of extended measuring time for signal averaging in stationary discharges. Frontend demands are similar to those of interferometers and the other microwave diagnostics. No insurmountable problems are expected. However, if retro-reflectors must be used inside the vessel, surface roughness caused by sputtering of CX neutrals should stay below a quarter wavelength to maintain the phase front of the probing beam. It seems not to be an issue if tungsten mirrors are used [60]. In addition thermal expansion could affect the perpendicularity of the mirror arrangement which must be preserved to better than $10 \operatorname{arcs}$ [155]. 


\subsection{Particle diagnostics}

Hydrogen, helium and lithium beams. Particle diagnostics cover a wide field of applications. In active diagnostics beams of $\mathrm{H}, \mathrm{He}$, or $\mathrm{Li}$ are injected into the plasma. After interaction with the bulk plasma, the emission of beam atoms excited by electron impact (beam emission spectroscopy, BES) or deuterons and impurities (charge exchange recombination spectroscopy, CXRS) can be investigated. Both thermal and energetic atoms are used. Information deduced is mainly on electron density and temperature in the edge region of the plasma $[158,159]$. While thermal beams are produced using nozzles, integrated into the first wall or the divertor tiles, high-energy beams of Li allowing for deeper penetration into the plasma need external sources and accelerators coupled via differentially pumped vacuum lines.

Problems in long pulse operation are the problems of spectroscopic diagnostics in general mainly connected with the front-end optical components. As with DNB operation, the beams will not be run continuously in steady state discharges. The optical systems used for observation can be protected by using shutters during the off-phases, lowering significantly the problems connected with thermal load and coating.

Heavy ion beam probe. Heavy high-energy singly-charged ions like $\mathrm{Cs}^{+}, \mathrm{Tl}^{+}, \mathrm{or}_{\mathrm{Au}^{+}}$are injected to probe the local plasma potential with the so-called heavy ion beam probe (HIBP) by measuring the energy change and the trajectory of the double-charged ion reaching the detector $[160,161]$. The diagnostic, important in particular for stellarators, can benefit from stationary long pulses by signal averaging of the usually small detector signals allowing in addition for cross-correlation fluctuation measurements. No specific long pulse problems are expected, however, HIBP measurements are not requested over pulse periods longer than a few $10 \mathrm{~s}$.

Charge exchange neutrals. The energy and/or momentum analysis of charge exchange neutral particles leaving the plasma (CX-NPA) gives information on the ion energy distribution in the plasma $[158,162]$. Operated together with a DNB allows for local measurements. As is common to all particle diagnostics, as well as for VUV spectrometers, an extension of the torus vacuum to the beam source and the detector is necessary compatible with its pumping requirements and, in case of BPX, with tritium handling [163].

No problems are reported with long pulse operation of neutron diagnostics to get information on the total yield, the neutron source strength and its distribution in space and time, and from neutron spectroscopy on the ion temperature. Details of neutron diagnostics essential in BPX are given elsewhere [164-167].

\subsection{Divertor diagnostics}

Divertor diagnostics are adapted to the parameter range expected in the plasma edge yet are basically not different to core plasma diagnostics, however, access is typically much more restricted demanding for sophisticated design solutions [168, 169]. The weight and the applicability of individual diagnostics might have somewhat been shifted. Besides probes and particle beams, dedicated Thomson-scattering or LIDAR systems are used as well as microwave and spectroscopic systems including bolometry and thermography. To accomplish safe divertor operation is most important in particular in long pulse high power discharges. Protection of the target tiles by monitoring its surface temperature with IR cameras and IR detector arrays with sufficient resolution conducting calorimetry by measuring flux and 
temperature changes of the coolant when passing through tiles must have highest priority. They must provide in real time signals for fast interlocks.

For long pulse operation divertor erosion monitoring is necessary. It can be done applying Speckle-interferometry allowing for sub-micron resolution [170]. 


\section{Radiation effects in burning plasma experiments}

The short introduction is based primarily on references $[12,63,171]$ and references therein. Radiation effects are expected small under long pulse conditions in TS, LHD, KSTAR, and W7-X even under D-operation conditions, since the annual neutron budget is limited to about $10^{19}$ neutrons. Radiation effects occurring under BPX conditions, which are of concern for diagnostic components in particular the plasma facing optical components, the cables of the magnetic diagnostics and the fibres used in spectroscopic systems are briefly reviewed in the following chapters. The environment as expected for ITER is taken as reference. Neutron fluxes, dose rates, CX-particle fluxes, and thermal radiation from the plasma as expected for the ITER standard scenario are given in [56]. It is the radiation flux, which is of concern in the early phases of a BPX. In later phases both flux and the integrated fluence are responsible for the radiation damage, which has built up. The radiation field will induce numerous different effects in the materials, however, the primary effects can be divided into different components, damage due to displacements of atoms within the solid state body, ionization and transmutation [171].

Displacements are caused by collisions between the neutrons and atoms or ions of the solid body lattice resulting in defects, which may aggregate to form macroscopic defect zones. Both metals and insulating materials are affected. Recombination can compensate for displacement effects in parts. The first wall displacement dose of ITER will be of the order of $10^{-6} \mathrm{dpa} / \mathrm{s}$. Total integrated dose might build-up to about 1 [172].

Ionization is caused in metals and insulators by particles and electromagnetic Gradiation from the burning plasma. Energy transfer due to ionization causes volumetric heating in metals. In insulators charge transfer effects dominate. Dose rate for ITER first wall will be about $2 \times 10^{3}$ Gy/s [3].

Transmutation products will build up with time modifying important material properties. Transmutation is due to nuclear reactions initiated by high-energy neutrons producing finally impurities in the materials, which can significantly alter their mechanical and electrical properties. As a result, materials perish and swell, and window transmission is degrading. In addition radiation induced luminescence occurs permitting the use of silica based optical components near to the plasma completely $[11,57,172,173]$. While stainless steel can withstand more than $1 \mathrm{dpa}$ and $10^{9} \mathrm{~Gy}$, insulators are far more affected becoming useless at orders of magnitude lower doses [10]. The quality of electrical insulation by ceramics is however, a key issue for diagnostics. Ceramic insulators are used in feedthroughs, connectors, mechanical supports, mineral insulated cables and their seals. They are used as substrates for bolometers and as holders for electrodes in other sensor devices like pressure gauges, which have to be mounted inside the vessel. Radiation tests have been conducted using fission irradiation sources to establish a database for ceramics, windows, optical fibres, mirrors and cables $[57,174,175]$. De-calibration of thermocouples due to transmutation effects are measured to be small [176].

\section{Plasma Facing Optical Components}

In long pulse high power nuclear fusion experimental devices spectroscopic diagnostics which aim at a reasonable field of view, face the delicate problem that the highly sensitive first optical element needs to withstand the extreme conditions in the vicinity of the plasma wall. 
This means for non-BPX experiments that one needs to find solutions which make them cope with high heat loads of $50 \mathrm{~kW} / \mathrm{m}^{2}$ up to a few $100 \mathrm{~kW} / \mathrm{m}^{2}$ in the most extreme cases. These heat loads mainly originate from the predominantly short wavelength radiation $(<6 \mathrm{~nm})$ emitted by the plasma which is absorbed within the first millimetre of any material $[177,178]$. However, under certain operation conditions, as discussed in chapter 6, also high levels of ECRH stray radiation can contribute significantly, but with very different absorption characteristics. Apart from the pure heat load problem, the PFOCs have to withstand heavy particle bombardment mostly by CX-neutrals, which leads to erosion, implantation and redeposition of elsewhere eroded material on these components. The demands on the optical components following the first plasma facing optical element, i.e. lens and mirror based relay systems or optical fibres, are less stringent, the main problems being connected to $n-$ and $\square$ radiation. While the various presently investigated solutions for entire spectroscopic observation systems have already briefly been presented and discussed in chapter 7.3, the following discussion will concentrate on the details of how the individual optical components are affected by the conditions of the environment they are used in.

\subsection{Windows}

\subsubsection{Thermo-mechanical effects}

Windows made of different optical materials so far were the common way to gain spectroscopic access to fusion plasmas. In short pulse machines in most cases fused silica or quartz windows were used because they combine a large spectral transmission range with good neutron compatibility. However, the poor thermal conductivity of these materials makes them unsuitable for use in high heat load locations in long pulse machines. As Table 2 shows the optimum material for nearly all applications is CVD diamond [179]. The window material is extremely expensive and diameters larger than $120 \mathrm{~mm}$ and $2.5 \mathrm{~mm}$ thickness, which would be particularly viable, have not been produced so far. The thermal conductivity has been found to decrease with increasing total fast neutron fluence $(\mathrm{E}>1 \mathrm{MeV})[180]$ but remains even at very high fluences of $10^{22} \mathrm{n} / \mathrm{m}^{2}$ of the order of a few $100 \mathrm{~W} /(\mathrm{m} \mathrm{K})$ and thus sufficiently high to allow efficient heat removal via edge fluid cooling $[181,182]$ as demonstrated with the ECRH gyrotron output window developed for W7-X and ITER [183].

The high heat load compatibility of window materials other than diamond as well as different concepts for active cooling of windows has been discussed in various studies [178, 184-188]. Two main types of actively cooled window designs have so far been tested. At CEA Cadarache small diameter sapphire windows $(\varnothing \sim 20 \mathrm{~mm})$ bonded to metal ferrules in different ways have been successfully exposed to maximum heat loads of up to $25 \mathrm{~kW} / \mathrm{m}^{2}$ over many heat cycles in the vacuum chamber of the Infrared Irradiation Test Facility IRIFA. A large number of these windows is now being used in the TS IR-thermography endoscopes for long pulse operation [189]. For W7-X a different design approach is being followed which avoids any form of bonding. The window is simply being pressed between two Helicoflex seals, with the window rim sticking out into a surrounding water cooling channel. This design aims at window diameters of up to $200 \mathrm{~mm}$. The design allows for free thermal radial expansion, thereby avoiding the build up of thermal stress, and for an easy exchange of windows when they have reached high transmission losses due to the steady build up of coatings during long pulse operation. Moreover, this design appears suitable for any type window material. A similar approach has been successfully demonstrated for a small CVD diamond gyrotron entrance window (diameter: $30 \mathrm{~mm}$ ) for power loads of up to $100 \mathrm{~kW} / \mathrm{m}^{2}$ at cw operation $[185,190]$. The design of actively cooled windows for W7-X has initially been tested with quartz and sapphire windows in a specially designed high heat load vacuum test 
chamber equipped with a Boralectric IR heater plate [105, 177, 191]. So far sapphire windows with an exposed diameter of $57 \mathrm{~mm}$ have sustained heat loads of up to $25 \mathrm{~kW} / \mathrm{m}^{2}$. An insufficient cooling of the IR heater facing metal construction of the window resulted in an edge heat source for the sapphire window via the Helicoflex seal and thereby in a centre to edge temperature difference of $\square \mathrm{T} \sim 200{ }^{\circ} \mathrm{C}$. For slightly higher heat loads and therefore larger temperature differences, thermal stresses led to cracking of the window as expected $[188,192]$, while without edge cooling $\left(\square \mathrm{T}=0{ }^{\circ} \mathrm{C}\right)$ no rupture occurred up to the maximum tested temperature of $310^{\circ} \mathrm{C}$. The fracture limit had been slightly raised to $\square \mathrm{T} \sim 260{ }^{\circ} \mathrm{C}$ by using a- instead of c-cut crystals since they don't suffer from a drastic loss of compressive strength with increasing temperature and are less prone to twinning on rhombohedral crystal planes [193]. The poorly cooled metal front plate is now being redesigned to become compatible with higher heat loads. The limitations with respect to maximum window diameters for given thicknesses for the different special materials required for the UV or IR spectral regions, have been investigated theoretically $[178,188]$. Recently also investigations into not commonly used materials like magnesium spinel $\left(\mathrm{MgAl}_{2} \mathrm{O}_{4}\right)$, which has an optical transmission similar to sapphire [194] combined with excellent swelling behaviour under neutron irradiation, or yttrium oxide $\left(\mathrm{Y}_{2} \mathrm{O}_{3}\right)$ have begun [186].

\begin{tabular}{|c|c|c|c|c|}
\hline Material & $\begin{array}{l}\text { Transmission } \\
\text { range, } \\
\mu \mathrm{m}\end{array}$ & $\begin{array}{c}\text { Thermal } \\
\text { conductivity at room } \\
\text { temperature, } \\
\mathrm{Wm}^{-1} \mathrm{~K}^{-1}\end{array}$ & $\begin{array}{c}\text { Melting } \\
\text { temperature, } \\
{ }^{\circ} \mathrm{C}\end{array}$ & $\begin{array}{c}\text { Stress limit } \\
\text { at room } \\
\text { temperature, } \\
\mathrm{MPa}\end{array}$ \\
\hline fused $\mathrm{SiO}_{2}$ & $0.16-3$ & $1.4[195]$ & 1715 & 38.25 \\
\hline quartz & $0.2-3$ & $6.2[195]$ & 1700 & 50 \\
\hline $\mathrm{CaF}_{2}$ & $0.14-12$ & $8.1[195]$ & 1418 & 37 \\
\hline $\mathrm{BaF}_{2}$ & $0.15-15$ & $11.7[196]$ & 1280 & 26.2 \\
\hline $\mathrm{MgF}_{2}\left(\operatorname{Irtran} 1^{*}\right)$ & $0.11-7.5$ & 14.9 [196] & 1255 & 50 \\
\hline $\begin{array}{c}\mathrm{MgF}_{2}{ }^{\mathrm{sc}} \\
\text { (single crystal) }\end{array}$ & $0.11-7.5$ & $21[196]$ & 1255 & 50 \\
\hline $\mathrm{ZnSe}$ & $0.5-22$ & $18.2[195,197]$ & 1550 & 55 \\
\hline ZnS (Irtran 2) & $0.5-13$ & $15.49[196]$ & 1827 & 97.1 \\
\hline $\mathrm{ZnS}\left(\mathrm{CVD}^{* *}\right)$ & $0.5-13$ & $17[196,197]$ & 1827 & 102.7 \\
\hline sapphire $\mathrm{Al}_{2} \mathrm{O}_{3}$ & $0.17-5.5$ & $46.06[195]$ & 2050 & $350-690$ \\
\hline $\begin{array}{l}\text { diamond } \\
\left(\mathrm{CVD}^{* *}\right)\end{array}$ & $0.12-10$ & $1900[195]$ & - & $1100-2500$ \\
\hline
\end{tabular}

*) polycrystalline MgF2: Irtran is a registered trademark of the Eastman Kodak Company.

**) CVD: chemical vapour deposition.

Table 2: Window material properties [188]

Failure due to shock heating [198, 199], which is expected for similar temperature differences between front and back side of a window, as for thermal stress failure, hasn't been of much concern in present day pulsed fusion experiments, which are mainly equipped with fused silica windows, which have a fairly high shock resilience [200]. The limit for sapphire is considerably lower and decreases with temperature. IR windows made of $\mathrm{ZnSe}$ already have a significant failure probability for thermal loads in excess of $1.5 \mathrm{MW} / \mathrm{m}^{2}$ in a $50 \mathrm{~ms}$ burst, as calculations and an accident on RFX demonstrated [200]. Therefore the risk of thermal shock failure due to sudden events needs to be investigated before allowing large windows made of 
materials with poor heat conductivity (see Table 2) to run up to high central temperature during long plasma pulses.

A further point that needs to be considered in the design of an optical system observing through an actively cooled window is that the radial temperature gradient in the window results in a radial variation of the refractive index due to its temperature dependence [179, 201]. The window thereby becomes effectively a gradient index lens with varying focal length during a long high power plasma experiment. The effective focal length of a $\mathrm{ZnSe}$ window can get less than $10 \mathrm{~m}$.

ECRH can also affect the heat load of any window as already discussed in parts in chapter 6 . Under certain operation scenarios high levels of ECRH stray radiation can build up. This can lead to arcing at sharp edges, but it will also be absorbed by any of the optical components [202]. The power fraction absorbed in the window material is determined by the loss tangent of the material and is given by $f=e^{[[(2 \square / \square) \tan \square] b}$, where $\square$ is the wavelength of the microwave radiation and $b$ the window thickness. The loss tangent $\square$ for different optical materials has been tabulated as a function of microwave frequency and window temperature [90].

Compared to the short wavelength plasma radiation which is nearly completely absorbed by any window, only a fraction of the mm-wave radiation is absorbed in a window. While sapphire absorbs only a small amount of $140 \mathrm{GHz}$ radiation at $200{ }^{\circ} \mathrm{C}(0.2 \%$ for a $10 \mathrm{~mm}$ thick window) $\mathrm{ZnSe}$ and $\mathrm{ZnS}$ windows have a 20 times larger loss tangent, leading to an absorption of $\sim 5 \%$ for the same window thickness and temperature. For an un-cooled $\mathrm{ZnS}$ or $\mathrm{ZnSe}$ vacuum window this can lead to temperatures in excess of $400{ }^{\circ} \mathrm{C}$ at which both materials start to decompose in an oxidising atmosphere, like air or oxygen. Under these conditions a $\mathrm{ZnO}$ film forms at the air side of the windows [203] affecting their transmission properties [204, 205], e.g. an uncooled ZnSe window in the W7-X ECRH stray radiation test chamber became opaque in the visible [92]. The relatively low power absorption of the ECRH stray radiation by the vacuum window means that the stray radiation can penetrate deep into the optical system, with the high risk of not only heating the optical elements but also being strongly absorbed by gaskets, optical fibres, plastics, ceramics or other materials. The installation of a screening micromesh at the window to prevent the stray radiation from penetrating into the system should be considered.

When designing a sapphire window for high heat load operation one needs to take into account that the emissivity of this material strongly varies with temperature. The fact that good absorbers are also good emitters means for the case of sapphire, which is only transparent for IR radiation up to about $4.5 \mu \mathrm{m}$, that the emittance depends in first approximation on the blackbody spectral power distribution at a given temperature. At room temperature $\sim 99 \%$ of the total exitance is emitted above $4.5 \mu \mathrm{m}$ and at $200{ }^{\circ} \mathrm{C}$ that figure still amounts to about $90 \%$. Therefore the assumption of an emissivity coefficient of $\square \sim 0.9$ ( $\square=1$ in totally opaque case) is a reasonable assumption for temperatures in this range. For higher temperatures the detailed temperature dependence of the total emission coefficient needs to be included in any window calculations based on finite element modelling (FEM). In many calculations, however, simply a conservative constant value of $\square=0.1$ is being assumed instead. Experimentally determined total emission coefficients have been reported for low temperatures, only for non transparent white $\mathrm{Al}_{2} \mathrm{O}_{3}$ for $24{ }^{\circ} \mathrm{C}(\square=0.94)$ and $93{ }^{\circ} \mathrm{C}(0.90)$ [206]. These values can be used as a first approximation since at such low temperatures a blackbody radiates predominantly in the non-transparent long wavelength region for sapphire. For temperatures between $730{ }^{\circ} \mathrm{C}$ and $1230{ }^{\circ} \mathrm{C}$ several normal, total emittance values have been published [207] (Figure 3), while for 500 to $1000{ }^{\circ} \mathrm{C}$ spectral emissivities, derived from 
measured normal incidence absorption coefficients (Figure 4), can be used to calculate total emissivities [202].

Values for intermediate temperatures need to be calculated from measured spectral absorption and transmission coefficients [202, 208-211] and the temperature dependent real part of the refractive index. For sapphire, however, only a single experimental thermo-optic coefficient, $\mathrm{dn} / \mathrm{dT}$, is given by the various manufactures. In this case temperature dependent Sellmeier equations can be used which satisfactorily characterise the thermo-optic coefficients of presently available optical materials [212].

\subsubsection{Radiation effects in windows}

In non-BPX long pulse high power experiments neither window transmission losses nor luminescence radiation play any role due to the fairly low path length (thickness) of these optical components, while in BPX experiments they cannot be used at all as first plasma facing optical components.

The neutron flux at the position where windows are foreseen in BPX experiments need to be lowered by shielding (labyrinth) to values below $\sim 10^{7} \mathrm{n} / \mathrm{cm}^{2} / \mathrm{s}$ [213]. Based on ITER R\&D, fused silica glass or quartz windows are recommended for spectroscopic systems for the visible region, and sapphire windows should be used for the IR region. Optical absorption in sapphire is relatively insensitive to Zradiation and only displacement damage associated with neutron irradiation induces notable absorption. Thus, under high-flux ionising-radiation, sapphire will have some advantages over fused silica. Irradiation of sapphire windows of 10 $\mathrm{mm}$ thickness with neutron fluences of up to $510^{23} \mathrm{n} / \mathrm{cm}^{2}(0.05 \mathrm{dpa})$ demonstrated that they can be used in the IR region from $850 \mathrm{~nm}-5 \mu \mathrm{m}$, while in the visible spectral region absorption losses of $10 \%(400 \mathrm{~nm})$ to $60 \%(600 \mathrm{~nm})$ have to be expected [214]. Radioluminescence is very strong in sapphire [213, 215-219] and will be a problem above 100 $\mathrm{Gy} / \mathrm{s}$ [172]. A very detailed review of possible window materials for fusion reactor diagnostics [220] came to the conclusion that for the spectral region from $350-2000 \mathrm{~nm} \mathrm{KU}-$ 1 quartz is the most appropriate material under the conditions expected for ITER $n$ - and $\square$ radiation. At fluences of $10^{22} \mathrm{n} / \mathrm{cm}^{2} \mathrm{KU} 1$ does not only retain its transparency but doesn't even suffer from radioluminescence as its intensity level is negligible compared to the expected bremsstrahlung intensity for the ITER ignition plasma regime and therefore will not affect any optical measurements of plasma parameters.

In the last years work concentrated on the UV spectral region from $200-350 \mathrm{~nm}$ where the high and low $\mathrm{OH}$ quartz glasses KU1 and KS4V [11, 221-223] and even more so sapphire $[219,223]$ show strong increase in absorption and luminescence at irradiation doses of $10-$ $130 \mathrm{MGy}$. By increasing the temperature the absorption of the KU1 window can be significantly reduced [222]. By rising the temperature from $35^{\circ} \mathrm{C}$ to $170{ }^{\circ} \mathrm{C}$ about a factor of three in transmission can be gained while surprisingly the absorption in KS4V increases by a similar amount [221]. More detailed studies revealed that the temperature dependent absorption of $\mathrm{KS} 4 \mathrm{~V}$ goes through a maximum at $120{ }^{\circ} \mathrm{C}$ [221]. Investigations of the photoluminescence of the $4.2 \mathrm{eV}$ band at $295 \mathrm{~nm}$ showed clear correlation with the $\mathrm{OH}$ content of the materials [224], increases with dose rate [225] and can be thermally quenched by rising the temperature above $150{ }^{\circ} \mathrm{C}$ [11]. A study of $\mathrm{CaF}_{2}$ irradiated by $14 \mathrm{MeV}$ neutrons $\left(1.119^{19} \mathrm{n} / \mathrm{m}^{2}\right)$ showed significant absorption peaks in the UV and visible spectral range [226]. Moreover, the ease with which colour centres are induced by either neutron or X-ray irradiation suggests that $\mathrm{CaF} 2$ is not a good final optic window for fusion experiment. Its 
behaviour for applications at wavelength below $200 \mathrm{~nm}$ has, however, not yet been studied. In summery, for operation temperature below $50{ }^{\circ} \mathrm{C} \mathrm{KS} 4 \mathrm{~V}$ seems to be the most suitable material for the UV region while above this temperature KU1 is more favourable.

The damage probability of laser windows increases due to n- and 5 -irradiation. However, at the dose rates and accumulated doses expected for non-BPX experiments, KU1 quartz glass and high purity sapphire are both suitable materials for which no increased damage probability need to be expected. Sapphire shows no notable changes in laser damage probability up to the investigated dose of $4.510^{4} \mathrm{~Gy}$, e.g. in W7-X a maximum dose of $10^{4}$ Gy in the course of 10 years can be expected. In case the laser window requires active cooling due to high thermal loads from plasma irradiation, sapphire with its high thermal conductivity will probably be the most appropriate material. In BPX experiments, however, where the laser windows are shielded from direct plasma radiation and thus high heat loads, KU1 presently seems to be the most appropriate choice since it has a considerably lower damage probability at high laser power densities. Tests on KU1 at power densities of up to $510^{16} \mathrm{~W} / \mathrm{m}^{2}$ and dose rates of $70 \mathrm{~Gy} / \mathrm{s}$ (ITER LIDAR expected: 10-100 Gy/s) even showed a reduction in damage probability with increasing accumulated total dose from $20 \mathrm{kGy}$ up to $10 \mathrm{MGy}$ compared to unirradiated windows [227]. Allowing $0.1 \%$ absorption, a $4 \mathrm{~cm}$ optical path length double window made of KU1 quartz could be used at a neutron fluence of $810^{20} \mathrm{n} / \mathrm{m}^{2}$ at the window location for $\sim 50000$ ITER discharges of $1000 \mathrm{~s}(\sim 12500 \mathrm{~h})$ duration, i.e. it would most likely not even be necessary to heat the window in order to anneal created colour centres [120]. By pre-irradiating the KU1 windows with doses of $\sim 20 \mathrm{MGy}$ at $200{ }^{\circ} \mathrm{C}$ one could remove any potential problem of ionising radiation induced laser absorption since this has been observed to result in a reduction of optical absorption at $500 \mathrm{~nm}$ by at least a factor of 10 [222].

\subsubsection{Contamination of diagnostic windows}

In the course of one operating period of short pulse fusion experiments of typically one year a build-up of a deposit on many windows leads to a significant deterioration of their transmission. Such a one year period, however, is comparable to just two days of long pulse operation like foreseen e.g. for the stellarator W7-X with up to $10 \mathrm{MW}$ of heating power for two pulses of 30 minute duration per day. This means that the growth of deposits on observation windows in high power long pulse experiments poses a serious problem, which needs to be carefully addressed. One has to take any measures possible to minimise the deposition rate, use blinds to shield the windows during the different stages of vacuum chamber preparation, develop special cleaning techniques and for some extreme locations even might have to consider methods for window exchange via vacuum locks.

The contamination of diagnostic windows originates from various physical processes, resulting in different avoidance and removal schemes. The main contaminants in fusion experiments, with carbon based divertor structures and wall protection, are different types of soft and hard layers of hydrocarbonates and metals.

When trying to minimise the build-up of metallic contamination on windows inside viewports due to sputtering by CX-neutrals geometry plays a significant role. In case of a port with a retracted diagnostic window, $\mathrm{CX}$ induced sputtering inside the port is expected to result in the build-up of a significant deposit on the window if the duct length $\mathrm{L}$ is larger than its diameter. This results from the fact that the ratio of the average flux of sputtered atoms from the duct wall onto the window surface is approximately six or even more times larger than the inverse flux from the window surface onto the wall [228]. For short ducts $(\mathrm{L}<<2 \mathrm{r})$ this ratio is less 
than one, i.e. the contribution from the walls to the deposit on the window becomes negligible. In this case the effect on the window depends on the rates of direct deposition and erosion due to physical and, possibly, chemical sputtering. Fairly unaffected operation can be expected for very long ports, $L / 2 r>(\cos \square)^{-1} \sim 10$ ( $\square$ : angle of maximum sputtering yield measured from normal to surface [229]) since in this case sputtering off the port wall takes place far from the window, so that the material will be re-deposited on the duct wall and hardly reach the window. The deposit growth on the window can be further reduced by installing diaphragms made of materials, which have a low sputtering yield, like Ta or W, inside the port. A disadvantage of far retracted windows is of course that they allow only for small viewing angles, but they are invaluable for cross calibration of other contaminated windows, via bremsstrahlungs measurements [230, 231]. On JET for example the bremsstrahlung seen with the vertical line of sight active CXRS observation system through an always clean window which is located about $2 \mathrm{~m}$ from the plasma, is being used to continuously cross calibrate the CXRS profile system which has a gradually deteriorating window very close to the plasma.

The measures described above to minimise the build-up of metal coatings are particularly important for the vacuum windows of high power laser radiation. Initial experiments with thin gold layers deposited by sputtering onto KU1 quartz led to a decrease in damage threshold for laser radiation by several orders of magnitude with decreasing layer thickness (peak around 5 $\mathrm{nm}$ layer thickness), implying a serious risk of window failure during operation at typical laser power densities of $10^{12}-10^{13} \mathrm{~W} / \mathrm{m}^{2}$ [232]. Fortunately, recent experiments with stainless steel (310 SS) sputtered onto KU1 quartz windows showed a much more favourable behaviour [233], with the damage probability increasing with layer thickness and with window failures for the largest investigated film thickness of $200 \mathrm{~nm}$ only occurring at laser power densities of $\sim 10^{15} \mathrm{~W} / \mathrm{m}^{2}$, which are a factor 100-1000 higher than e.g. the power densities at the vacuum windows of the JET Thomson scattering system [234] or those foreseen for the ITER systems [120, 235-237]. A problem which could potentially become important at required power densities is the observed increase in damage probability with increasing window temperature observed for layer thicknesses of $70 \mathrm{~nm}$ at $400{ }^{\circ} \mathrm{C}$ [233], but only if it would increase significantly with layer thickness (not yet investigated). This is of no concern for the ITER Thomson diagnostics since there the vacuum windows will be located deep inside the labyrinths where they are not subject to high heat loads and where the neutron fluence is sufficiently reduced so that annealing of created colour centres through high window temperatures will not be required [120].

In fusion machines with high heat load carbon components, the build-up of brownish hydrocarbon films on the windows is regarded as having the more detrimental effect on window transmissivity [238]. Physical sputtering, chemical erosion and chemical sputtering of carbon by hydrogen isotopes lead to a release of hydrocarbons which are redeposited all around the machine, even in shaded regions [239]. Laboratory experiments have shown that the structure and physical properties of a-C:H layers depend sensitively on the deposition conditions [240]. One can roughly distinguish two forms of a-C:H films, so called hard, diamond-like films and soft, polymer-like films [241]. They can be distinguished by their mechanical properties. Hard films are very scratch resistant while soft ones can be easily scratched off. The hard, amorphous layers have a large hydrogen content (H/C $\square 0.4)$ and form only under the impact of impinging ions with energies larger than $50 \mathrm{eV}[240,242]$, while the soft layers, containing equal amounts of hydrogen and carbon $(\mathrm{H} / \mathrm{C}=1)$, form at low ion energies $(<50 \mathrm{eV})$ [240] typical for divertor plasmas, e.g. average kinetic energy of impinging ions on the JET Mark IIa divertor surface $\sim 30 \mathrm{eV}$ [243]. Particularly thick soft deposits (up to $40 \mu \mathrm{m}$ ) with high hydrogen content $(\mathrm{D}+\mathrm{T}) / \mathrm{C}=0.7$ were found on the water 
cooled louvre at the entrance of the inner divertor pump duct shadowed from the plasma already after a short operation period of JET in 1996 [244, 245], which indicates that diagnostic observational access to the divertor plasma from these regions, as e.g. foreseen in W7-X, will most likely require the integration of a window cleaning technique. Analysis of samples from different machines, cleaning by thermal annealing and scratch tests, suggests that predominately soft, polymer-like films are deposited on in-vessel components and windows [239, 243, 244, 246, 247].

A further way to distinguish between soft and hard a-C:H layers is via spectral reflectance measurements [248] which not only allow to measure film thicknesses from $10 \mathrm{~nm}$ up to the $\mu \mathrm{m}$ range, but also to determine the complex refractive indices $\underline{n}=\mathrm{n}+\mathrm{i} \mathrm{k}$ of a layer [249, 250]. It was found that soft polymer-like layers have a characteristic refractive index of $\mathrm{n} \sim$ $1.57\left(\mathrm{k} \sim 1.510^{-3}\right)$ while for dense hard layers values of up to $\mathrm{n} \sim 2.4(\mathrm{k} \sim 0.13)$ have been observed, with the refractive index being a good measure of the hydrogen content of the films $[240,242]$. The measurement technique requires a continuous light source, a few optical fibres and a micro-spectrometer. It can be integrated into many optical diagnostics, particularly easily, of course, into fibre optic based systems.

Time resolved deposition measurements with the quartz microbalance (QMB) technique have demonstrated the strong dependence of film growth on discharge type in various machines [245, 251, 252]. Measurements by Skinner [251] with a couple of back-to-back installed QMB crystals, located $77 \mathrm{~cm}$ from the plasma at the end of a $\mathrm{L}=38.5 \mathrm{~cm}$ long tube $(\mathrm{L} / \mathrm{r} \sim=$ 4) located about half way down from the torus midplane, show high deposition rates on the plasma facing crystal, resulting in a $120 \mathrm{~nm}$ thick hydrocarbon film already after $200 \mathrm{~s}$ of accumulated plasma operation. Interestingly, at this remote location a few disruptions reduced the film thickness by $80 \mathrm{~nm}$. A further frequently observed phenomenon is a step-up in layer thickness just in the first one or two shots of the day and a slow continuous rise in deposition between discharges, possibly due to evaporation of polymer films after each discharge, followed by deposition in the inter-pulse period, as postulated [243]. A tenth of the deposition has even been found on the rear facing crystal, suggesting that also optical components not facing the plasma directly might have to be shielded by shutters and will require frequent cleaning when running high power long pulse experiments.

Detailed investigations particularly into the chromatic transmission of predominantly hydrocarbon coated windows have been undertaken in the field of Thomson-scattering diagnostics since the wavelength dependence leads to significant errors in derived temperatures, but of course also density measurements $[238,246]$. The measured spectral transmission losses of a TFTR window [238] were found to be very similar to samples coated in laboratory glow [253] and ECR discharges in $\mathrm{H}_{2} / \mathrm{CH}_{4}$ gas mixtures. Based on windows used in JT60-U during 1992-1995, a simple polynomial description for the wavelength dependent attenuation by a film of thickness $d$ has empirically been derived [246]. It can be used to describe chromatic transmission of a coated window

$$
\begin{aligned}
& \mathrm{T}(\square)=\mathrm{T}_{0} \exp [-\square(\square) \mathrm{d}], \\
& \text { with } \square_{\mathrm{fit}}(\square)=0.0439-9.9210^{-5} \square+6.2310^{-8} \square^{-2} .
\end{aligned}
$$

On JET the chromatic transmission losses made it necessary to clean the observation windows approximately every 4 weeks by laser blow-off [254].

Even though it is known from laboratory experiments that a higher $\mathrm{H}$ content as well as a higher temperature and ion energy results in a higher erosion yield and that net 
erosion/deposition depends on the dynamic state of the surface during interaction with the plasma [239], the subject of erosion of window surfaces has not been discussed in any fusion diagnostic papers so far. The impact of erosion on diagnostic mirrors for future fusion devices like ITER, where windows cannot be used at all as first plasma facing optical component, however, is a major research subject. The reason why erosion of window surfaces hasn't posed a problem in any fusion experiment so far might be, that perhaps they are always deposition and not erosion dominated due to their naturally slightly more retracted installation inside ports. Erosion, however, is potentially dangerous for laser launching windows of Thomson-scattering diagnostics since erosion driven roughening of window surfaces would lead to increased laser absorption resulting in window failure within few laser pulses. Not only direct erosion but also the exchange of oxygen by hydrogen in an about $1 \mu \mathrm{m}$ thick surface layer of laser entrance windows, through CX losses of fast hydrogen isotopes from the plasma, increases the laser vacuum entrance window damage probability significantly. Tests on a KS4V quartz sample irradiated with a dose of $510^{16}$ high energy $\mathrm{H}^{+}$ions $(55 \mathrm{keV})$ revealed a monotonic increase in optical absorption from IR to the UV which could lead to a local increase in temperature due to laser absorption in the $1 \mu \mathrm{m}$ hydrogen implantation layer [255]. This is expected to have a higher probability for laser damage than material deposition. An in-situ removal of such a layer by laser ablation is, of course, not possible and thermal annealing at $450{ }^{\circ} \mathrm{C}$ unfortunately even further degrades the window surface. It is therefore important to assess the particle spectrum and expected fluences of the energetic $\mathrm{H}$ atoms at the location of laser entrance windows. The $\mathrm{OH}$ content of laser entrance window materials seems not to play an important role, as the almost identical damage behaviour of high and low $\mathrm{OH}$ quartz glasses, KU1 (OH content: 800-900 ppm) and KS4V (OH $<0.2 \mathrm{ppm})$, showed, which both are very resistant to laser damage [233].

\subsubsection{Mirror and window cleaning techniques}

In long pulse machines in-situ cleaning of PFOCs will be unavoidable. The development of suitable techniques which can be integrated into the diagnostic view ports has been identified as high priority topic within the ITPA Topical Group on Diagnostics. While finding in principle suitable techniques is already difficult, their integration into the diagnostic ports is often even more challenging.

Photo-cleaning. Various techniques like photo-cleaning, thermal annealing or plasma etching are presently being discussed [256], but only laser cleaning of Thomson-scattering light collection windows using the diagnostic laser has actually been applied on nuclear fusion experiments [246, 254, 257]. Full transmission recovery can already be achieved with few pulses at laser energy densities of $0.3 \mathrm{~J} / \mathrm{cm}^{2}$. With a typical beam diameter of $\sim 4 \mathrm{~cm}$ at this energy density, cleaning of an entire window area is possible with a small number of steering steps [254]. Single pulse laser blow-off, requiring $2 \mathrm{~J} / \mathrm{cm}^{2}$, can be performed below the safe energy density level of $5 \mathrm{~J} / \mathrm{cm}^{2}$ given by lens makers and well below the damage threshold found experimentally for 500 laser pulses of $50 \mathrm{~J} / \mathrm{cm}^{2}$. At this energy density sweep speeds of $\sim 6 \mathrm{~cm}^{2} / \mathrm{s}$ can be realised, allowing for the cleaning of a $30 \mathrm{~cm}$ diameter windows in 7 minutes [257]. Metal coatings, however, might pose a problem. While stainless steel (310 SS) evaporated onto KU1 quartz windows could easily be removed by laser cleaning, the same layers being sputtered onto the window could not be removed with this technique at all [233].

Apart from lasers also the use of other short pulse ultra high intensity irradiation sources like flash- or pinch-lamps are being considered for photo-cleaning [256]. A removal rate of 2.6 $\mathrm{m}^{2} / \mathrm{h}$ for a $7 \mu \mathrm{m}$ soft a-C:H film with a flash-lamp $\left(3.4 \mathrm{~J} / \mathrm{cm}^{2}, 5 \mathrm{~Hz}\right)$ has been reported and 
removal rates of $100 \mathrm{~m}^{2} / \mathrm{h}$ for $50 \mu \mathrm{m}$ film thickness are believed to be feasible with commercially available $10 \mathrm{~J} / \mathrm{cm}^{2}$ flash-lamps operating at $500 \mathrm{~Hz}$ [258]. Technically problematic, however, is that in-vessel access with a manipulating arm, placing the light source right in front of the components to be cleaned, is required. In places where the available space allows the integration of steering optics near the plasma facing window, fibre optics can be an easy means to deliver sufficiently high power laser or flash lamp light bursts for window cleaning.

Various processes, like sublimation, vaporisation, thermo-mechanical shock, photo-chemical decomposition or acoustic shock can contribute to photo-cleaning. The dominant process can be chosen to some extent by the selection of source wavelength, power, pulse length, etc., which means that an optimum method for given contaminant and substrate materials could be selected.

Cleaning by thermal annealing. Experiments on soft and hard a-C:H films, similar to the coatings found on windows in fusion experiments, which were produced in RF and ECR discharges, respectively, have demonstrated that at least the soft films can efficiently be completely removed by thermal annealing [241]. Figure 5 shows that in vacuum a soft film of $300 \mathrm{~nm}$ thickness can be evaporated completely by heating a window to temperatures of about $400{ }^{\circ} \mathrm{C}$ for approximately 1 hour. While heatable high temperature (up to $550{ }^{\circ} \mathrm{C}$ ) viewports with fused silica windows are commercially available (Thermionics ClearView ${ }^{\mathrm{TM}}$ ), solutions which allow alternating operation of cooling and heating, during and after long pulse experiments, respectively, still need to be developed in particular, of course, for other window materials. Thermal annealing unfortunately cannot be used to remove any hard layers. Increasing the temperature only leads to a release of hydrogen and progressive graphitisation of the remaining carbon layer. The increased layer thickness is attributed to a release of intrinsic mechanical stress [241, 247]. As Figure 6 shows, removal of hard layers by thermal annealing requires an air vent of the machine. Cleaning of an ASDEX-Upgrade window in air at $650 \mathrm{~K}$ worked much faster than removal of a hard layer from a coated sample of the same thickness and under the same conditions, indicating that the main contaminant accruing on the windows in fusion experiments is a soft polymer-like substance, which can easily be removed by thermal annealing. In long pulse experiments a hard layer, nevertheless, might gradually build up due to the bombardment of the soft films with high energy CX neutrals. In order to avoid an air vent, provisions for other cleaning methods, like photo-cleaning, need to be taken, which most likely need not be applied daily, but at regular intervals. The gradual build up of a hard layer could be monitored by spectral reflectance measurements via the refractive index as described above.

Cleaning by plasma etching. A further cleaning technique presently being studied for its possible suitability is plasma etching by inductively coupled RF plasmas using gas mixtures of argon and oxygen [259, 260]. In such discharges charged particle bombardment leads to oxidation of the a-C:H layers, thereby forming $\mathrm{CO}, \mathrm{CO}_{2}$ and $\mathrm{H}_{2} \mathrm{O}$, which can be easily pumped. However, there is well founded concern that due to the use of oxygen the vessel condition will get lost. This would be avoided by localised low power ECR discharges operating with pure hydrogen. First tests on the cleaning ability of such discharges in the RIKEN ECR-2 device, using $50 \mathrm{~W} \mu$-wave discharge in $910^{-4}$ mbar of pure $\mathrm{H}_{2}$, were promising [253]. 


\subsection{Mirrors}

Mirrors can be used with advantage in long pulse experiments since active cooling can be provided much more easily than in case of windows. To avoid the elaborate development of actively cooled windows as conducted at W7-X, at KSTAR mirrors will be used throughout when long pulse operation with pulse lengths exceeding $10 \mathrm{~s}$ is envisaged. In future BPX the use of mirrors is mandatory. Their behaviour as PFOC under the harsh conditions of a fusion reactor is being studied since many years both theoretically and in experiments $[60,261]$. The mirrors are affected mainly in the volume by n-radiation, at the surface by erosion due to CX atoms bombardment and by coating of re-deposited sputtered wall material. Long pulse exposure experiments have been conducted and are still ongoing at devices like TS, TEXTOR, JET, NSTX, T-10, and LHD [262, 263]. In these experiments erosion and deposition are being studied. The behaviour of plasma facing materials under intense thermal loads as occurring during disruptions are discussed elsewhere [264].

\subsubsection{Radiation}

As has been reviewed before, the lifetime of all refractive components exposed to reactor grade plasmas is very short. Therefore all diagnostics that use UV, visible or IR radiation view the plasma via mirrors. Heating by UV and X-rays at ITER $1^{\text {st }}$ wall might be up to 500 $\mathrm{kW} / \mathrm{m}^{2}$ and neutron volumetric heating up to $8 \mathrm{MW} / \mathrm{m}^{3}$, which must effectively be cooled away under steady state conditions. The neutron flux causes besides heat in particular atomic displacement, which results in modifications of the material, i.e. decrease of heat conduction, swelling, and embrittlement [60]. However, irradiation shows little effect on the reflectivity of metallic mirrors made of $\mathrm{Cu}, \mathrm{W}, \mathrm{Mo}$, and stainless steel up to conditions of $10 \mathrm{dpa}$ [265]. They survive in the extreme hostile environment and maintain good optical performance. About $50 \%$ of all ITER diagnostics use in-vessel mirrors as front-end plasma facing component. Wavelengths span from $5 \mathrm{~nm}$ in XUV spectroscopy to $119 \square \mathrm{m}$ for interferopolarimetry and to about $5 \mathrm{~mm}$ for ECE and coherent scattering. The tolerance in surface quality, which is acceptable, strongly depends on the wavelength used. The relative change in reflectance $R / R_{0}$ at wavelength $\Pi$ and average roughness of dimension $d$ can be expressed by $\exp \square(4 \square d / \square)^{2}$. Material selection should provide $d<\Pi / 50$ for long term conditions to stay below $10 \%$ reflection loss [60].

\subsubsection{Erosion}

The erosion of near plasma facing components and the vessel wall is an old topic in fusion research [266] causing impurities on one hand and modifying the surface of mirrors on the other. The erosion of frontend components is almost exclusively caused by the bombardment of $\mathrm{CX}$ atoms and ions in the energy range up to several $\mathrm{keV}$ from the plasma giving rise to erosion and even damage when simultaneously irradiated with high power laser pulses of the LIDAR system as a result of synergistic effects. Ion bombardement experiments conducted with stainless steel mirrors show a clear dependence on the ion energy [60]. The reflectance at $600 \mathrm{~nm}$ is reduced to $75 \%$ at a sputter depth of $3 \square \mathrm{m}$ for $1.4 \mathrm{keV}$ ions. The same depth caused by $0.3 \mathrm{keV}$ ions results in only $5-7 \%$ reflectance loss. While destroying phase front characteristics, surface roughness has a stronger influence on mirrors for image transmission compared to those just used for collecting and reflecting light. The first are much more sensitive. It could be shown experimentally that the resolving power of optical systems is strongly reduced with increasing surface roughness [60]. Experiments with $\mathrm{Cu}$ showed a 
reflectance reduction by $30 \%$ at $650 \mathrm{~nm}$ and $1.5 \square \mathrm{m}$ thickness of sputtered layer, while the resolving power of the system is reduced to about $10 \%$ (Figure 7 ).

The situation is much improved using monocrystalline material. While polycrystalline $\mathrm{W}$ at $650 \mathrm{~nm}$ shows a monotonic decrease of reflectance with thickness of sputtered layer, monocrystals are almost unaffected. Monocrystalline Mo and $\mathrm{W}$ turned out to be resistant to erosion under long-term sputtering conditions. The reflectance and the degradation depends on the orientation of the crystal surface. Existing technology allows for the production of Mo mirrors of up to about $10 \mathrm{~cm}$ in diameter and up to $5 \mathrm{~cm}$ in case of W. For larger mirrors facetted structures have to be used. Instead of single crystalline material also metal films on metal substrates seem to be possible.

Metal films of 1-10 \m thickness on metal substrates give the possibility to choose the mirror material and to fabricate larger sizes. Examples are $\mathrm{Be}$ on $\mathrm{Cu}, \mathrm{Cu}$ on $\mathrm{Cu}$, and $\mathrm{Rh}$ on various substrates. $\mathrm{Rh}$ on polycrystalline $\mathrm{Cu}$ substrate is a good candidate where the reflectance is not very high but stays rather constant during bombardment.

Calculations of flux and mean energy of CX atoms to the first wall in ITER result in about $10^{19} / \mathrm{cm}^{2} \mathrm{~s}$ and $350 \mathrm{eV}$ respectively. Under these conditions single crystalline $\mathrm{W}$ and Mo mirrors have lifetimes sufficient for ITER i.e. $10^{4}$ shots of $1000 \mathrm{~s}$ duration. In case small entrance apertures in front of the first mirror are used, the CX flux is reduced by orders of magnitude to levels where no significant effects are expected.

Due to the wavelength dependence discussed before, the reflectance of mirrors for FIR at 100 $\square \mathrm{m}$ and the microwave diagnostics is not significantly reduced, even at mean roughness of 2$3 \square \mathrm{m}[267]$.

\subsubsection{Coating}

As already discussed in the context of windows, the coating of the plasma facing surface by eroded material transported and accumulated at the mirror might strongly deteriorate their optical quality. Mirrors are more affected than windows since the light passes two times through the coating film. It seems that no good models exist to calculate the transport of eroded material to predict the rate of deposition of contaminants on the PFOC surface. The optical properties and the thickness of the film strongly depend on the experimental conditions, the mirror material and the location of installation in the machine [61]. Experiments have shown that thin films $(10 \mathrm{~nm})$ of $\mathrm{C}-\mathrm{H}$ or Be can strongly modify the reflectance of mirrors as well as its polarization characteristics [268, 269]. The latter is of concern in particular for the important MSE diagnostics. Theoretical studies were carried out to determine the effects of $\mathrm{Be}$ and $\mathrm{C}$ deposits onto $\mathrm{Au}$ mirrors foreseen [108]. The optical properties are affected by any of theses deposits, however, $\mathrm{C}$ induces stronger optical changes on the $\mathrm{Au}$ mirror than Be for the same thickness of the deposits. Polarization changes expected for MSE are significant. Measurements on plasma-exposed mirrors indicate the need for in-situ calibration systems monitoring the changes of the mirror optical properties [270]. Carbon based films of up to $50 \mathrm{~nm}$ thickness have been found after exposure of stainless steel mirror samples in LHD. The properties are strongly depending on the location ranging from hard films mainly consisting of $\mathrm{Fe}(40 \%), \mathrm{C}(40 \%)$, and $\mathrm{O}(10 \%)$, which were difficult to

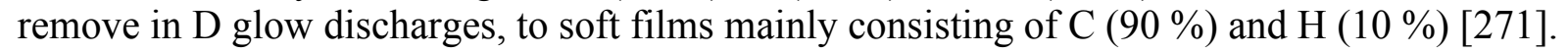
The deposition strongly depends on the individual poloidal position in the torus and the temperature of the surface. The development of cleaning techniques is essential. Different methods can be envisaged, the chemical erosion by ions from low temperature deuterium plasmas or the removal by laser ablation as discussed before. Both methods were successfully applied to clean stainless steel mirrors from $\mathrm{C}$ films. 


\subsubsection{Long pulse experiments}

Long time exposure experiments were conducted at TS with a number of mirrors located on the high field side of the plasma vessel about $140 \mathrm{~mm}$ away from the last closed flux surface [262]. The samples are mounted parallel to the toroidal direction. The results are assumed typical and are briefly summarised. Monocrystalline Mo, polycrystalline stainless steel and $\mathrm{Cu}$ samples were used and exposed in total to about $26000 \mathrm{~s}$ of pulse length with a cumulated energy of about $18 \mathrm{~kJ} / \mathrm{cm}^{2}$ wall load. In the experimental period lasting about one year also wall conditioning procedures like glow discharges in $\mathrm{He}$ or $\mathrm{D}_{2}$ have been conducted as well as boronization.

The analysis after exposure showed almost no changes of the roughness of the Mo and stainless steel samples, however, the $\mathrm{Cu}$ sample was clearly affected. The surface roughness was increased by about a factor of 10. Chemical analysis using SIMS showed carbon deposits enriched with hydrogen, deuterium, boron, and oxygen with a thickness of about $12 \mathrm{~nm}$.

The optical reflectivity was only slightly reduced as a consequence of both, erosion and absorption, probably mainly by the latter.

The reflectivity loss measured is slightly higher for stainless steel since its surface is stronger affected by erosion. $\mathrm{Cu}$ was deteriorated most. It showed clear modifications concerning surface roughness, net-erosion, and reflectivity. The effects are most pronounced in the near UV. Almost no effects are found for FIR wavelengths.

Large polycrystalline metal mirrors of Mo and W have been exposed in the SOL plasma of TEXTOR mounted on inclined target holders [269]. Reflectivity degradation has been determined in the wavelength range of 250 to $2500 \mathrm{~nm}$.

\section{Fibres}

Other than in the case of windows, radiation effects in optical fibres can have a significant effect already in non-BPX experiments since the optical materials are exposed to the $n$ - and $\square$ radiation over comparatively long path lengths. The effect of radioluminescence in fibres, however, not only depends on the local induction of the luminescence in the fibres but also on their transmission properties for the induced radiation. Luminescence and loss of transmission in fibres has experimentally been evidenced not only in the D-T experiments on JET and TFTR, where they were of course most prominent, but also already at levels of D-D fusion yields of many present day fusion experiments [272, 273]. The early studies of the effect of ionising radiation on optical fibres and various possible mitigation strategies have been explored mainly for fission reactors applications, often using radioactive ${ }^{60} \mathrm{Co}$ sources. In these studies fibres with pure silica cores where investigated, since they were found to be least susceptible to radiation induced attenuation in the $800 \mathrm{~nm}-1.5 \mu \mathrm{m}$ region used for optical communications [274]. This holds for both, short times following pulsed irradiation as well as long times after steady state exposure. The radiation sensitivity of these fibres depends on their $\mathrm{OH}$ contend, with high $\mathrm{OH}$ fibres (sometime called 'wet' fibres) being least affected by radiation losses [274] in this spectral regime.

Since both $\mathrm{Si}$ and $\mathrm{O}$ have extremely low thermal neutron cross sections, it is mainly $\square$ radiation from the (n, $\square$ ) reactions of the neutrons produced in high power NBI heated discharges which affect the silicon fibres. An expected enhanced luminescence due to the ${ }_{14}^{28} \mathrm{Si}(n, p)$ reaction with a threshold at about $4 \mathrm{MeV}$ [272], which is above the $2.45 \mathrm{MeV}$ birth energy of D-D neutrons has, but well with the $14 \mathrm{MeV}$ energy of D-T neutrons, however, has 
not been observed during the DTE1 experiments on JET, where the luminescence has been found to just scale with the neutron production rate with no deviation due to the $14 \mathrm{MeV} \mathrm{D}-\mathrm{T}$ neutrons [273].

Radiation induced absorption (RIA). So far most of the work has concentrated on high purity fused silica fibres with silicon polymer (usually designated as plastic glad fibre (PCS), $\left.\mathrm{T}<150^{\circ} \mathrm{C}\right)$ or aluminium $\left(\mathrm{T}<350^{\circ} \mathrm{C}\right)$ cladding. Radiation induced opacity has both transient (time scale seconds) and permanent components [272]. Assuming that the transmission $\mathrm{T}$ is related to the defect density $n_{D}$ and the fibre length $L$ by

$$
T=e^{\square k_{1} n_{D} L}
$$

and that the defect density $n_{D}$ as a function of time is

$$
\frac{\square n_{D}}{\square}=\square \frac{n_{D}}{\square_{D}}+k_{2} F
$$

where $\mathrm{F}$ is the Gflux which causes the defects and the k's are constants, one can determine a finite defect lifetime $\square \mathrm{b}$ [272]. By fitting this model to the fibre transmission recovery phase following a high power NBI heating phase in TFTR $(F=0)$, a typical defect self-annealing time of approximately $\mathrm{b}_{\mathrm{b}} \sim 1.5 \mathrm{~s}$ was derived. Knowing this figure, the experimentally determined time dependent transmission loss could well be described as $T(d B)=k_{3} L F \square_{D}\left(1 \square e^{\square t / \square_{D}}\right)$ by making use of the above two equations.

In a similar experiment on JET during the DTE1 experiment with tritium, a fibre transmission recovery time of about $7 \mathrm{~s}$ has been observed in an unheated PCS fibre loop located near a main horizontal port [230]. Recovery times at room temperatures obviously can vary over a fairly large range from milliseconds, using e-beam pulses, to $10-100 \mathrm{~s}$ using ${ }^{60} \mathrm{Co}$ sources [274]. By in-situ thermal annealing at temperatures of up to $400{ }^{\circ} \mathrm{C}$ it was possible to reduce the transmission losses by more than a factor of 100 in aluminium clad radiation sensitive low-OH fibres while in high-OH fibres no transmission losses at all could be detected any more, neither during the TFTR [275] nor the JET DTE1 experiments $\left(\mathrm{T}_{\text {fibre }} \sim 250{ }^{\circ} \mathrm{C}\right.$ at the plasma facing end as well as along the lead fibre) $[230,276]$. However, the variability in transmission losses between fibres drawn from different raw glass blocks can be quite substantial. For example in one sample of 'dry' fibres a temperature increase from $20{ }^{\circ} \mathrm{C}$ to $420{ }^{\circ} \mathrm{C}$ led only to an improvement in fibre transmission by a factor of 7 and in case of high$\mathrm{OH}$ fibres drawn from different batches considerable variation in fibre transmission at room temperature was found. It is therefore strongly advisable to only use fibre compensation loops drawn from the same raw material batch [275].

In-situ measurements have been performed on silica and sapphire fibres $(400 \mu \mathrm{m})$ in the UVvisible spectral range from 200-800 $\mathrm{nm}$ with gamma rays (dose rate $0.092 \mathrm{~Gy} / \mathrm{s}$, total dose $34.5 \mathrm{kGy}$ ) and protons (dose rate $100 \mathrm{~Gy} / \mathrm{s}$, total dose $1.8 \mathrm{MGy}$ ). For the silica fibres, with increasing total Girradiation dose from 0.33 to $12.22 \mathrm{kGy}$ not only the attenuation in the 220 $350 \mathrm{~nm}$ spectral region increases, but also a clear widening of the overall absorption peak due to a shift from the $248 \mathrm{~nm}$ peak (corresponding to the B2a colour centre) towards the $318 \mathrm{~nm}$ peak (associated to the bound chlorine colour centre) is observed. In case of the proton irradiation the overall UV absorption peak shows a fairly similar behaviour. [277]

For BPX plasmas investigations have shown that fibres can be used with neutron fluences of up to $10^{13} \mathrm{n} / \mathrm{cm}^{2}$ for wavelength higher than $300 \mathrm{~nm}$. The behaviour of optical fibres is 
excellent in the IR region (low absorption and low radio luminescence). Only at $1.39 \mu \mathrm{m}$ an $\mathrm{OH}$ vibration band can lead to strong absorption for polymer clad KS4V fibres which, however, can be avoided by using metal cladding [278]. R\&D indicates that the radiationinduced loss could be less than $10 \mathrm{~dB} / \mathrm{m}$ in the wavelength region of $800-1200 \mathrm{~nm}$ for some optical fibres, with a fast neutron fluence of $10^{25} \mathrm{n} / \mathrm{m}^{2}$ and ionising radiation dose of $10^{10} \mathrm{~Gy}$.

Radiation induced luminescence (RIL). Luminescence in high purity fused silica fibres is largely unpredictable. The band around $650 \mathrm{~nm}$ is believed to arise from a non-bridging oxygen (NBO) defect in the silica matrix and depends strongly on manufacturing technique, $\mathrm{OH}$ content and radiation damage history while the blue band centered at $450 \mathrm{~nm}$ is less well understood [272, 279] (Figure 8). In low fluence experiments like TFTR or JET both effects are thermally quenched at fibre temperatures above $150{ }^{\circ} \mathrm{C}$ resulting in radio luminescence being dominated by Cerenkov radiation at elevated temperatures. While the luminescence yield due to Cerenkov radiation cannot be reduced by fibre heating, it can be reduced proportional to $1 / \mathrm{d}$ (d: fibre diameter) by using larger diameter fibres (Figure 9) [275]. The reason for this is believed to be that the radiation interaction length is proportional to $\mathrm{d}$ while the throughhput of the fibre and hence of the sensitivity of the optical system increases as $\mathrm{d}^{2}$. Therefore the ratio of radioluminescence over throughput of the fibre falls as $1 / \mathrm{d}$. As Figure 9 shows the fibre size scaling does not depend on $\mathrm{OH}$ content, fibre type or manufacturer.

Figure 8 shows that the luminescence signal observed at JET near $523.5 \mathrm{~nm}$ in an un-heated PCS fibre can be expected to solely originate from Cerenkov radiation. Therefore the luminescence linearly scales just with the neutron production rate and is insensitive to the energy of the plasma neutrons, i.e. $2.45 \mathrm{MeV}$ neutrons from DD-reactions and $14 \mathrm{MeV}$ neutrons from DT-reactions during the DTE1 experiment produce identical results (Figure 10). The results show that even in high power deuterium discharges the $Z_{\text {eff }}$ determination via measured bremsstrahlung can be significantly affected. Especially for clean, low $Z_{\text {eff }}$ plasmas an installation of parallel blind fibres or fibre loops for measuring luminescence alone is already mandatory for $\mathrm{Z}_{\text {eff }}$ measurements in DD plasmas with high neutron rates [273].

At high fluence of $\mathrm{n}$ - and Gradiation the observed luminescence signals show rather complex dependences leading to sometimes large data scatter and non-linear responses due to a dynamic competition between temperature recovery and displacement damage procedures. Moreover, the Cerenkov radiation, which is dominant in silica fibres, decreases with increasing wavelength while the absorption goes the other way leading to an apparent peak [11].

Luminescence measurements on sapphire show strong narrow bands at 605 and $695 \mathrm{~nm}$ and a very broad band ( $\sim 100 \mathrm{~nm}$ wide) at $450 \mathrm{~nm}$ increasing with increasing proton irradiation dose from 1.32 to $3.32 \mathrm{MGy}$ [277]. From the viewpoint of radio luminescence, fused silica has an advantage over sapphire as a window material.

Radiation hardening. Two different techniques based on hydrogen and fluorine doping have been applied to step index pure silica core fibres of $200 \mu \mathrm{m}$ diameter with a core cladding ratio of 1.1 to 1.2 . The hydrogen treatment of the KU-H2G fibre results in a clear suppression of the broad $2 \mathrm{eV}$ absorption band centred around $620 \mathrm{~nm}$ which develops in silica due to the formation of non-bridging oxygen hole centres (NBOHC) and peroxy radicals (POR). This leads to a clear improvement in the radiation induced absorption of this fibre over the two fluorine doped fibres and the low-OH KS4V fibre (Figure 11 and Figure 12) [173]. The KU$\mathrm{H} 2 \mathrm{G}$ fibre underwent a pre-irradiation followed by a molecular hydrogen treatment at high temperature $\left(>80^{\circ} \mathrm{C}\right)$ and pressure $(>100$ bar $)$. Whether this technique can also be successfully applied to large core fibres of $600-1000 \mu \mathrm{m}$ still needs to be demonstrated. The 
high level of radioluminescence in fibres still is of concern and a way to reduce it hasn't been found yet. The hydrogen doped fibre even showed a slightly higher luminescence than the undoped one and the highest value of all 5 tested fibres. Operation at high temperature (300 ${ }^{\circ} \mathrm{C}$ ) resulted in a further reduction of the transmission losses in KU-H2G fibres during irradiation but also in an apparent rise of luminescence due to improved transmission of the Cerenkov emission induced by the Gradiation. It should be noted, that both fluorine doped fibres suffered from very high optical bending losses and that one of them showed a quickly degrading transmission and did not survive the reactor test.

Radiation induced changes in numerical aperture. If rays with larger angles were attenuated more than others due to radiation induced transmission losses this could affect the optical filling of a spectrometer and thereby change the instrumental function resulting in slightly skewed Doppler profiles or small changes in velocity measurements. Such an effect could result from radiation induced damage to the cladding and other boundary effects causing losses during internal reflection which depend on the number of reflections and the angle of the emerging light ray $\square$. Under this model assumption the fibre transmission could be described in first approximation as

$$
T(d B)=\square \frac{10 L \square}{d n} \square R
$$

with $\mathrm{L}$ fibre length and $\mathrm{n}$ refractive index. By fitting this model to experimental data the change in the effective reflection coefficient $\square R$ can be derived. For an investigated $22 \mathrm{~m}$ long, $1 \mathrm{~mm}$ diameter PCS fibre a value of $\mathrm{dR} \sim 310^{-6}$ has been found. This effect is small enough not to require serious changes in optical matching, as long as the total losses are kept low by e.g. fibre heating [275].

Removal of fibre transmission losses by photo-bleaching, which has been reported on earlier [280], seems not to be effective for un-doped silica core fibres and had only a marginal effect on Ge-doped fibres in the wavelength region of lowest loss increase $(800-1200 \mathrm{~nm})$ [281].

\section{Cables}

As briefly introduced in chapter 8, radiation effects may change mechanical and electrical properties of wires and cables used for diagnostic purposes. The ITER R\&D program has established a database and has initiated tests since these effects are the most critical issue in BPX.

Mineral insulated (MI) coax cables are typically used in radiation environments [282]. In the field of ionizing radiation they must accommodate radiation induced currents and voltages representing additional noise, introducing in particular measuring errors. The error signals occur between inner and outer conductors of coax cables and as a differential voltage through asymmetries in the radiation field in otherwise symmetric electrical circuits. This is of serious concern for sensitive magnetic diagnostics. A number of radiation induced (RI) effects have been identified. The following four are of importance for diagnostic cables: radiation induced conductivity (RIC), radiation induced electrical degradation (RIED), radiation induced electromotive forces (RIEMF), and radiation induced thermoelectric sensitivity (RITES). 
Radiation induced conductivity means an increase of electrical conductivity of an insulating mineral material in the presence of ionizing radiation [174]. Electrons are excited from the valence band into the conduction band followed by trapping into levels near to the conduction band from where they can thermally be excited into the conduction band again. The response therefore depends on the dose rate present and on the temperature of the irradiated material. For given cable, RIC increases as the square of the cable length. While qualitatively well understood [283], experimental tests need to be conducted to determine the complex response quantitatively $[171,174]$. Materials as $\mathrm{MgO}, \mathrm{Al}_{2} \mathrm{O}_{3}$ have conductivities smaller than $10^{-6} \mathrm{~S} / \mathrm{m}$ for relevant dose rates up to $10^{3} \mathrm{~Gy} / \mathrm{s}$ and at temperatures of $450{ }^{0} \mathrm{C}$ [171]. Since magnetic diagnostics for ITER require a conductivity of better than $10^{-6} \mathrm{~S} / \mathrm{m}$, RIC should not be a main problem for these diagnostics.

Radiation induced electrical degradation. In contrary to RIC, RIED seems to be not fully understood and is still controversially discussed [171, 284]. It is similar to RIC since it is affecting also the insulator conductivity yet with additionally an electric field present. The electrical conductivity is increased, induced by defects created by the radiation field in the presence of an electrical field. The main difference to the former is that the induced degradation builds up and is conserved depending on the total fluence applied. The degradation of the insulating properties occurs for dose rates of about $10^{2} \mathrm{~Gy} / \mathrm{s}$, at temperatures above about $200{ }^{\circ} \mathrm{C}$ and electric fields higher than about $50 \mathrm{kV} / \mathrm{m}$.

Radiation induced electromagnetic forces is the generation of spurious currents, which can lead to error signals in the measurement [57, 285-289]. The currents can create voltages between the core and the sheath of a coax cable depending on the insulation resistance of the cable and its external load. Expected currents are $1 \square \mathrm{A}$ in maximum. Mineral insulated cables consist of one or two metallic core wires surrounded by the insulator and a sheath made of stainless steel. Current creation between core and sheath occurs under the influence of intense $\mathrm{n}$ - and Gradiation where different nuclear reactions are initiated resulting in the injection of energetic electrons into the insulating material. The electrons are emitted with some delay as the result of the decay of unstable isotopes formed after neutron capture, or they are produced prompt due to direct $\square$ processes creating free electrons via photo-effect, Compton scattering, or pair formation [286]. Time constants of the delayed processes can vary between minutes and hours. The total charge released depends on the cable materials, on the materials in the cable surrounding, on the energy spectrum of the radiation as well as the temperature of the exposed material. Complex temperature dependencies are reported [289].

Due to the sensitivity of the RIEMF induced currents on the surroundings, the induced current in a coiled cable is different from the current in an uncoiled one of the same length. Monte Carlo codes have been developed to predict the currents [286]. For a typical coiled $1 \mathrm{~mm}$ mineral insulated cable the induced current under Girradiation is about

$5 \mathrm{pA} /(\mathrm{m} \mathrm{kGy} / \mathrm{h})$. The use of copper leads to higher currents with lower time constant compared to stainless steel or Inconel. $\mathrm{Cu}, \mathrm{Al}$, and $\mathrm{Mn}$ should be avoided [287].

In case of poor common mode rejection of differential amplifiers used in magnetic diagnostics, RIEMF can cause also in two core wire circuits error signals indistinguishable from true signals. On the other hand asymmetries in the radiation field can cause differential voltages as well. Experiments conducted at the JMTR fission test reactor showed during longterm integration large drifts in symmetric arrangements of up to $40 \mathrm{mVs}$, being far beyond ITER requirements. 
Radiation induced thermoelectric sensitivity. In-situ measurements of the voltages across mineral insulated prototype ITER coils at the same test facility showed that they are mainly originating in thermoelectric effects called RITES [290]. The thermal sensitivity develops gradually during irradiation and might be caused by transmutation and lattice damage varying along the wire, introducing variations of the thermo-power of the wire material. The necessary temperature gradients to generate a net-voltage are provided by nuclear heating in a nonuniform radiation field [291]. These results suggest that RITES may dominate the other radiation-induced effects occurring in diagnostic cables. The effect could be reduced placing the coils in uniform radiation flux regions and by cooling.

\section{Magnetic Measurements}

After giving a brief overview on magnetic diagnostics in chapter 7, in this chapter the challenges of the magnetic measurements are discussed in detail with respect to long pulses. The most commonly used information by other diagnostics is the geometry of the plasma making different diagnostics at different locations comparable. Magnetic measurements are also essential for the determination of the magneto-hydrodynamic (MHD) properties of the plasma and can be basically divided into two different parts. The first is related to the determination of MHD equilibrium. These signals determine the magnetic fluxes and local magnetic field values in steady state or at least slowly varying in time (slower than any MHD response). The magnetic equilibrium configuration is required for the precise control of current profiles, for establishing advanced scenarios and edge configuration for particle exhaust and low local power fluxes. The other part is related to the detection of MHD activity, which is a fast variation of the plasma overlaid on the equilibrium state (linear approximation). The magnetic signals can give information on spatio-temporal evolution of plasma instabilities and, thus, provides essential means for mitigation or avoidance of them.

The diagnostics used for such measurements are typically based on inductive sensors. With increasing duration of the discharges demands on intrinsically steady state capable diagnostics arise. Some alternative sensors are given in the literature (see section 12.4).

\section{1}

\section{Plasma parameters and sensor requirements}

\subsubsection{Equilibrium}

The requirements on equilibrium reconstruction are different for stellarators and tokamaks. The poloidal field in tokamaks is generated mainly by the toroidal plasma current. In order to know the position, shape and the safety factor of the flux surfaces, precise knowledge on the poloidal flux distribution and local field values is needed. These quantities can be directly measured outside the plasma column only. The solution of the Grad-Shafranov equation leads to the distribution of the poloidal flux. Information necessary for the active plasma control and stabilization can be derived [292-294]. Taking into account nonlinear effects of the magnetized transformer core, accurate solutions are more difficult to obtain. In long pulse tokamak experiments the need for real time equilibrium reconstruction grows in order to achieve optimum performance [295]. In particular the shape and position of the last closed flux surface is of major importance during long pulse operation to avoid thermal overload of 
limiting structures. Long pulse control of the plasma position based on magnetic measurements has been performed successfully at the TS tokamak [296].

At first glance, stellarators do not have such strong demands, since the main poloidal flux is generated externally and good flux surfaces exist even without any plasma current. This vacuum magnetic field configuration can be well analyzed by field line tracing using electron beams [297]. The flux surface geometry and rotational transform is modified by the finite plasma pressure and the toroidal plasma current profile, even in the case with vanishing net current. A hybrid system based on a quasi-axisymmetric configuration (NCSX) is presently under construction, where a significant fraction of the rotational transform is obtained by the bootstrap current [298]. In such systems, the demands on equilibrium reconstruction are much closer to tokamaks. The advantage of knowing the vacuum field configuration is reduced by the much more complex MHD equilibrium computations due to the 3D shaped plasma geometry, since e.g. in highly 3D shaped plasmas it is difficult to find toroidal flux contours, on which a flux loop could deliver a good measure of the poloidal flux. In this respect, NCSX has the strongest requirements on equilibrium reconstruction and prediction, because MHD equilibria have to be computed in $3 \mathrm{D}$ with a significant contribution by plasma currents and have to be calculated as function of time for equilibrium evolution prediction [299]. Even in modern stellarators of different types like the Heliotron/Torsatron, quasi-symmetric systems like QAS, QHS and QPS, and stellarators of the Heliac and Helias type, the need for real time equilibrium reconstruction grows in case the advantages of long pulses are exploited using more advanced feedback loops. Such feedback loops may comprise an intelligent divertor operation or simply needed to protect first wall components from thermal overloading.

In steady state discharges, the needs for tokamaks and stellarators merge, since intelligent plasma control and, consequently, automated data analysis is mandatory for advanced scenarios in both systems. The final goal of all diagnostics for steady state experiments is to have the analyzed data immediately available with lowest possible latency. This holds in particular for the equilibrium reconstruction, since many other diagnostics rely on this basic information. The most prominent information is the mapping between laboratory and magnetic coordinates, which allows in an integrated data analysis for the combination of diagnostics observing at different locations. The term real-time denotes different time constants for stellarators and tokamaks. For tokamaks, feedback loops have shortest time constants in the order of several milliseconds [22] for plasma stabilization purposes, whereas in stellarators plasma control time constants are in the order of seconds, given by current diffusion and L/R time constants [19]. The equilibrium reconstruction latency is planned to be of the order of $100 \mathrm{~ms}$ for $\mathrm{W} 7-\mathrm{X}$.

The following parameters obtained by magnetic measurements are essential for the determination of the plasma equilibrium [23, 282].

The net toroidal plasma current $\left(I_{p}\right)$ is typically measured by Rogowski-coils enclosing the plasma column poloidally. One important design criterion of this coil is a homogeneous winding area density, which makes the current measurements independent on the current distribution or position.

The loop voltage $\left(U_{\text {loop }}\right)$ is measured by a loop along the toroidal coordinate. Ideally, this loop is located inside the plasma in order to get the electromagnetic force $(\vec{\square} \square \vec{E}=\square \dot{B}$ by the ohmic transformer) driving the plasma current. The loop yields essential information on all devices with an ohmic transformer. 
The poloidal flux distribution outside the plasma needed for the plasma shape reconstruction is determined by loops following contours of constant poloidal flux. In 2D axi-symmetric devices these loops are simply toroidally circular. This kind of loop is difficult to design in case of highly $3 \mathrm{D}$ shaped plasma configurations, since iso-contours of the poloidal flux usually differ from the vessel shape and may change with the magnetic vacuum configuration. This signal has to be time integrated.

Moments of the toroidal current profile like the internal inductance $\left(L_{i}\right)$ and the centroid of $I_{p}$, can be derived by local magnetic field values $(\boldsymbol{B})$ measurements in both normal $\left(B_{n}\right)$ and poloidal tangential $\left(B_{s}\right)$ direction. These fields are recorded by virtue of localized pick-up coils, with sufficiently high winding area for maximal raw signals and, consequently, minimal integration errors.

The plasma stored energy $\left(W_{p}\right)$ is obtained by diamagnetic loops measuring directly the change in toroidal flux. This loop ideally encloses only the plasma region which is, however, usually not realizable and the loop also detects a significant contribution of the flux changes in the vacuum region. Hence, compensation loops are required measuring the flux change in the vacuum region only. Typically, a linear combination of the diamagnetic and compensation loops reduces the response to vacuum field changes.

The Pfirsch-Schlüter currents (PS-currents) are obtained by sets of saddle loops, mounted on top and bottom of the plasma vessel. The dipole structure of the PS-current produces a vertical flux intersecting the saddle loops. It contains information on the Shafranov-shift in combination with equilibrium reconstructions.

The pressure anisotropy, which is considered to be essential for a precise equilibrium reconstruction in case of discharges with strong ICRF- or NBI-heating, can be derived from a comparison of saddle loop and diamagnetic loop signals, since the latter one depends on the perpendicular pressure $p_{\square}$ only and the saddle coil signal on the total pressure $p_{\square}+p_{\|}[300]$. Maximum information is obtained, when the diamagnetic loop is aligned such that no vertical flux by the PS-currents intersects its cross section. In the tokamak case, anisotropic pressure has been derived from equilibrium reconstructions including an appropriate model taking into account the q-profile as measured by MSE [301].

Accuracy studies for ITER equilibrium reconstructions have shown, that the plasma shape at the main plasma wall gaps can be reconstructed with an accuracy of $1 \mathrm{~cm}$ or better when the noise level of the pick-up coil signal is less than $1 \%$ based on an artificial neural network (ANN) or function parametrization (FP) reconstruction. It can be relaxed to $3 \%$ in case of an EFIT reconstruction [23, 282, 302]. The plasma current measurements have been specified to be accurate to $1 \%$ or at least $10 \mathrm{kA}$ absolute in the low current range $(<1 \mathrm{MA})$. Similar requirements on the magnetic diagnostics are reported from TS [303]. Here, the claimed plasma position of below $1 \mathrm{~cm}$ requires an absolute measurement uncertainty below $1 \%$. In terms of plasma shape and position uncertainty, an accuracy of $1 \mathrm{~mm}$ has been reported [296]. More details on the accuracy requirements emerging from equilibrium reconstruction analyses can be found in [304-307].

In the average, an accuracy of $1 \%$ turns out to be a typical value envisaged for magnetic diagnostics on tokamaks. In case of stellarators, less information on the required accuracy is reported in the literature. This is due to the nature of the equilibrium reconstruction, where the modifications by the plasma can be considered as a perturbation on the flux surface geometry. The reconstruction of the equilibrium including the current profile by the external magnetic measurements is an ill-posed problem [295, 308, 309]. Thus, the quality of any equilibrium 
reconstruction is very sensitive to inaccuracies as introduced by noise, stray fields and systematic errors due to signal integration imperfections (section 12.3). The current profile determination for W7-X based on 80 NEMEC-equilibria revealed, that almost 4 moments of the current profile $I_{m}=\square(\square) \square^{m+1} d \square$ can be determined, provided the signals of the poloidal field measurements are accurate to $1 \%$. At this level, $I_{2}$ has already $50 \%$ uncertainty.

\subsubsection{Fluctuations}

Diagnostics for magnetic fluctuations are intrinsically long pulse compatible, since no signal integration is required. The main challenge is the proper integration of the magnetic sensors into the steady state capable first wall elements. Magnetic fluctuations are usually detected by Mirnov-coils, where the term fluctuation defines the time varying part of the magnetic field that is not attributed to a MHD equilibrium state. This typically covers all kinds of plasma oscillations like Alfvén-modes, pressure driven modes, tearing modes and sawteeth which even in disruptions may be analyzed using dedicated pick-up coils able to measure fast field changes. The essential parameters are mode frequencies and the poloidal and toroidal mode numbers for an efficient description of the spatio-temporal structure of the perturbation. A lower boundary for the range of oscillation frequencies is around $1 \mathrm{kHz}$, since the induced signal is proportional to $f$ and the winding area is naturally limited by the high frequency requirements of the coil. However, at TCV, a setup is used, where the fast amplifier, the low frequency and the integrator electronics are combined in order to detect magnetic signals from $\mathrm{DC}$ to frequencies above $10 \mathrm{kHz}$ [307]. In long pulse devices, this approach is presumably not usable, since the long pulse integrators are fed with error currents flowing from one input and protection stage to the other. The fluctuations with lowest frequencies are locked modes, which can induce disruptions in tokamaks [310]. They are caused by already small error fields of the order of $B_{\text {err }} / \mathrm{B}=10^{-4}$. They can be detected by particularly arranged saddle coils, which are sensitive to low order poloidal harmonics and the $n=1$ harmonic [23]. The highest frequencies, beside those in turbulent fluctuations, are observed in toroidally coupled Alfvén gap modes, the mirror induced Alfvén eigenmodes in stellarators [311]. Corresponding frequencies can exceed $1 \mathrm{MHz}$ in plasmas at fusion relevant densities.

Basically three problems have to be solved in long pulse experiments for pick-up coils: First, maximum distance between sensor and plasma in order to cope with the steady state heat fluxes. Any multipole structure of order $m$ decays, however, very fast with the distance $1 / r^{(m+1)}$ and limits the spatial poloidal resolution. The second problem is caused by the fact that more conducting material of the protecting shields is in proximity to the pick up coil, changing the signal phase by inductive and capacitive coupling. The third problem is the automated and reliable analysis of mode numbers, essential for plasma analysis and control. Due to axi-symmetry, this is less crucial for the toroidal mode number analysis in tokamaks and simple phase correlation algorithms can be used. In poloidal direction in a tokamak and in both poloidal and toroidal directions in stellarators the algorithms for mode number identification are more complex due to the geometric oscillation of the flux surface. Additionally, if phase corrections have to be applied on the transfer function of the system consisting of a conducting environment, the coil, an amplifier and a filter, the implementation of analysis algorithms becomes much more complex, also due to envisaged streaming capabilities for real time analysis. 
The requirements on the sensor given by the diagnostic objectives and the steady state capable design are often quite different, as discussed in the previous section. To avoid induction from eddy and halo currents and external fields, magnetic sensors should be as close as possible to the plasma and distant to conducting materials. With respect to steady state needs, however, the sensor should be as distant as possible to the plasma edge and should have sufficient heat conduction to actively cooled components. In practice, a good compromise has to be found.

Depending on desired time resolution, skin times of the plasma vessel and halo currents flowing in the vessel, pick-up coils and flux loops may be located in- or outside the plasma vessel. If both versions can be implemented, information on currents flowing in the vessel and the conducting surroundings can be derived. The advantage of installing the sensors outside is the inherent protection against any power fluxes like plasma radiation, convective plasma fluxes, arcs and ECRH stray radiation (see chapter 6).

A further long pulse aspect of the sensor design is related to the signal integrator problem. The integrator imperfections can be made less relevant, if the raw signal of the pick-up coils and flux loops is large. This can be achieved by a large winding area, which is obtained by increasing the number of windings and the cross sectional area. However, a low total resistance is beneficial for the subsequent integrator stage since it reduces the influence of creeping currents on the integrator circuit board as well as radiation induced currents in cables (see chapter 11). Increasing the number of windings increases the length and reduces the cross section of the wire. Consequently, an increase of the pick-up coil cross section is preferred, because under the assumption of a dense winding the internal resistance as function of number of windings $N$ and cross section area $A$ of a pick-up coil is $R_{i} \sim N^{3} \sqrt{A}$. The signal is $U \sim N A$ thus the current flowing into the input stage is in the optimum case $I=U / R_{i} \sim \sqrt{A} / N^{2}$. This current has to be compared with the creeping and radiation induced currents for the assessment of the coil and flux loop design. Although long pulse devices become larger, the space available for magnetic sensor is still restricted. This fact is caused by the need to have steady state enabled first wall elements, blankets in reactor experiments and cryogenic insulations outside the plasma vessel.

The design goal of maximizing the raw signal is limited considering the fastest field changes expected. Fast field changes, as they occur in the fast magnetic field ramp down for protecting the quenching superconductor or during plasma current disruptions, can easily induce voltages of the order of several $\mathrm{kV}$ in coils that are appropriate to detect $1 \%$ signal change reliably in a $1000 \mathrm{~s}$ discharge due to the high winding number.

Interestingly, the requirements on equilibrium coils are similar in spherical tokamaks as reported for NSTX [312] and MAST [313]. The Rogowski-coils have to fit into the limited space inside the centre stack and some additional Rogowski-coils for halo and eddy current detection are placed behind protection tiles at operating temperatures of $600{ }^{\circ} \mathrm{C}$. The coils, withstanding such high temperatures, are made of ceramic webbing as mandrel material and ceramic insulated wires and provide sufficient structural flexibility in case of NSTX. MAST uses Thermaleze wires, a combination of polyimide and polyester insulation, wound tightly on stainless steel tubes. Similar material is in use for the TS equilibrium coils [296]. At lower temperatures $\left(120^{\circ} \mathrm{C}\right)$, Teflon has been used for the main Rogowski-coil as mandrel material 
and polyimide insulation for the wire itself. KSTAR prefers to use mineral-insulated $(\mathrm{MgO})$ cables throughout for the magnetic equilibrium diagnostics, even for the Rogowski-coil [314]. This $\mathrm{MgO}$ insulated cable is wound tightly on a $9.5 \mathrm{~mm}$ stainless steel tube and can therefore cope with temperatures far above the specified $250{ }^{\circ} \mathrm{C}$. In $\mathrm{W} 7-\mathrm{X}$, polyimide insulated wires are used, which are wound tightly in a double layer onto stainless steel tubes of $5 \mathrm{~mm}$ diameter. Ten of these single Rogowski-elements are placed side by side outside and inside the vacuum vessel for an overall flat coil shape of less than $8 \mathrm{~mm}$ in height and $80 \mathrm{~mm}$ in width allowing to adapt the shape to the complex 3D shape of the plasma vessel.

Flux loops, comprising saddle loops and voltage and poloidal flux loops, are preferably made of MI cable. It is reported to use 2 and $3 \mathrm{~mm}$ MI cable for the ITER voltage and saddle loops, respectively [23, 282], and $1.55 \mathrm{~mm}$ MI cable is envisaged for the KSTAR flux loops [314]. MAST uses for flux loops single wires inside a thin walled stainless steel tube fixed by high temperature PTFE sleeves[313]. Tore Supra uses for the flux loops stainless steel shielded coaxial cable with Nextel ceramic fibres as insulation and a $1.13 \mathrm{~mm}$ thick $\mathrm{Cu}-\mathrm{Ni}$ wire [296]. W7-X is equipped with saddle coils, which are made of $3 \mathrm{~mm}$ MI cable consisting of 6 inner copper wires. These wires are connected to twisted $1 \mathrm{~mm}$ MI coax cable in a special vacuum tight and brazed T-connector. In this way, a closed 6-turn saddle loop could be mounted onto the highly $3 \mathrm{D}$ shaped vacuum vessel.

Diamagnetic loops are more critical in finding a design, which allows to have the main loop close to the plasma but to withstand high thermal loads. The response time of a diamagnetic loop depends on the compensation of fluxes caused by the external magnetic field and the fluxes caused by eddy currents. It is of advantage to have two loops inside the plasma vessel, one, which is very close to the plasma boundary and a second loop being located closer to the vessel wall. Taking the difference, a measure of the fluxes caused by vessel currents is obtained. The proper compensation leads to faster diamagnetic signals. The flux in the periphery of the plasma column can also be obtained by compensation loops. Fine compensation of single diamagnetic loops with a resultant diamagnetic bandwidth of $10 \mathrm{kHz}$ has been demonstrated for TCV [315], being, however, a rather complex task.

In a long pulse experiment the setup of a double diamagnetic loop is only feasible, if enough space is left between the vessel wall and the elements of the protecting first wall or if loops with low winding numbers are used as in TS, where a two turn loop made of $1.05 \mathrm{~mm}$ thick cable has been realized [296]. In the first case however, a loop design with large enough winding area and large compensation signal levels can be realized, which has its merits in particular when analogue pre-compensation has to be used. The latter is necessary if the dynamic range of the subsequent integrator is limited as for analogue integration techniques. In W7-X, the diamagnetic loop and its compensation coils are made of ribbon polyimide insulated cable consisting of 25 single wires. A 2- and 4-turn version is installed leading to 50 and 100 turns in total, respectively. The maximum induced voltage integral yields values of 5 $\mathrm{Vs}$ in plasmas at the highest expected $\langle\square>$ of about $5 \%$. The ribbon cable is fixed in two stainless steel side plates, which are formed to fit into the vessel shape. The whole construction protrudes by $11 \mathrm{~cm}$ into the vessel and, consequently, the shape of the first wall is adapted by a roof made of clamped graphite tiles [316] covering the loop. A design for this roof has been found, which can withstand heat fluxes of up to $500 \mathrm{~kW} / \mathrm{m}^{2}$ without any active cooling of the loop casing itself.

The discrete coils for local measurements of the magnetic flux density are usually less critical in a design for long pulses. KSTAR uses MI cable tightly wound on two distant ceramic rods [314]. Higher frequencies are damped out by the stainless steel tube of the MI cable used. However, for equilibrium information a bandwidth of $1 \mathrm{kHz}$ seems sufficient. Tore Supra uses 
alumina for the coil former and polyimide insulated $\mathrm{Cu}$ wire of 0.3 or $0.5 \mathrm{~mm}$ thickness [296]. These coils can withstand temperatures of up to $220^{\circ} \mathrm{C}$. In W7-X, the poloidal fields induced by plasma currents are low compared to those of a tokamak. Thus, rather long coils with a large winding area have been designed, which are almost identical to the W7-X Rogowskicoils. These coils measure the contour integral over B on a short poloidal section. The partitioning has been adapted to the poloidal field structure caused by internal plasma currents.

In general, connectors should be avoided for the equilibrium diagnostics where possible, because they reduce the reliability of the system and introduce further sources of contact voltages or even thermoelectric voltages, in case the pins are not made of the identical metal or alloy. In particular, thermoelectric voltages turned out to be one of the major problems of the conventional magnetic equilibrium diagnostics. Careful material selection is mandatory, in particular at wire connections. Tore Supra could avoid the use of any connections inside the plasma vessel by prolonging the coil wire up to the feedthrough [296]. This is of great advantage, since the strongest temperature differences occur inside the plasma vessel during steady state discharges.

Magnetic fluctuation measurements employing Mirnov coils do not have any intrinsic problems with long discharges. The sensor design, however, is directly affected by the conducting surrounding of the coil, which can not be avoided in particular in long pulse experiments. For a high spatial resolution, Mirnov-coils should reside close to the plasma edge to identify large poloidal mode numbers. High toroidal mode numbers are easier to detect, since the toroidal circumference is larger than the poloidal one. The mode number analysis is essential for identifying MHD activities. A precondition for the reliable analysis is to avoid frequency dependence in the transfer function of the coil in the desired bandwidth range or at least to know the influence of the conducting environment [317]. Such effects have also to be considered in large devices like ITER. The high frequency coils $(10-500 \mathrm{kHz})$ are located inside the poloidal gap between the shield modules [23]. The estimate of the field attenuation has been made on the theoretical basis of a waveguide.

In W7-X basically two different setups of Mirnov-coils exist. In the region of the highly loaded wall elements graphite tiles clamped on $\mathrm{CuCrZr}$ heat sinks are used. Mirnov-coils using AlN ceramic formers with grooves for the wires are placed between the heat sinks and thermally contacted and mechanically supported by clamps which are mounted on the cooling pipes. AlN is an insulator but has a very high heat conduction of $180 \mathrm{~W} / \mathrm{Km}$, which is half the value of copper. The heat conduction capabilities, however, degrade with neutron fluence [175]. The edges of the heat sinks and graphite tiles are aligned to the Mirnov-coil axis for avoiding phase changes in the transfer function. The second region is covered by water cooled stainless steel panels of $5 \mathrm{~mm}$ thickness, which are only loaded by plasma radiation. These panels are already rather distant to the plasma edge and electromagnetic damping behind these panels is simply too large for frequencies above $1 \mathrm{kHz}$. Thus, the Mirnov-coils in this region are placed on top of the panels with a graphite cap for protection against plasma interaction. The AlN former thermally couples the graphite cap to the stainless steel panels by virtue of an inter layer graphite foil. The design optimization has been performed for both thermal and electro-dynamical behaviour employing finite element calculations. 
Even in future long pulse experiments, inductive sensors seem to be the predominant method for the determination of magnetic fluxes and flux densities. Since only time derivatives of these quantities are recorded, stable electronic integrators are a prerequisite for discharges of duration $>1000 \mathrm{~s}$. In literature, several different types have been presented which intend to be stable enough for such long pulses.

The different error sources may influence the measurement of magnetic fluxes [282]. The most direct error source is noise coming from the electronic circuit of the integrator. The electronic noise can be minimized by careful circuit design. A common feature to all semiconductors is the noise below a frequency of $10 \mathrm{~Hz}$, which increases with $1 / \mathrm{f}$ [318]. It is, however, questionable, whether this noise is really of concern in analogue integration circuits, since so far, nothing has been reported with respect to long pulse integrators. Low frequency fluctuations of the plasma could also disturb the flux measurement, which is of relevance for fast feedback control for plasma stabilization. This problem can be solved reasonably by signal averaging in space and time as suggested in [282]. A major difficulty is the careful design of the connectors and the connection of the signal cables to the circuit of the integrator. At these contacts, variations of the contact potential and the thermoelectric effects can cause significant signal drifts. In particular the thermoelectric voltage reacts on temperature changes along the signal chain, which may occur at the backend of the system, i.e. temperature changes on the circuit board of the integrator in different operation states (signal integration or automatic offset correction at different output voltage levels) or at the front end of the system, when pick-up sensors and flux loops suffer thermal load by the plasma. Concerning the printed circuit boards, care has to be taken for leakage currents, since the input voltage offsets ( $\left.\mathrm{U}_{\text {off }}\right)$ have to be in the order of $100 \mathrm{nV}$ for $0.1 \mathrm{mVs}$ integration error after $1000 \mathrm{~s}$ of operation. Thus, typical on circuit voltages of $10 \mathrm{~V}$ can cause significant drifts if, e.g., the humidity of the air changes.

Several different approaches for stabilizing integrator operation can be found in literature. One important distinction is the integration method, i.e. analogue or digital. This property determines the dynamic range of the integrator. In analogue integrating circuits the dynamic range is limited by the output stage, which cannot exceed the voltage level of the power supply. The digital integrator typically performs conversion to digital values at the input voltage level and integrates by digital signal processing within a dynamic range determined by double precision values. On the other hand, analogue integration provides faster response of the output signal, whereas signal processing has some latency. Additionally, digital integrators are more complex due to the computer environment and the software involved and, hence, could be considered less reliable.

Classical analogue integrators as given in textbooks [319] are not suited for long pulse integration due to the temperature dependent $U_{\text {off }}(T)$ of typical operational amplifiers. Since the change of $U_{\text {off }}$ is usually slowly varying in time, automatic drift compensation has been added to such integrators (Figure 13a). This drift compensation is based on the idea to add an additional negative $U_{\text {comp }}=-U_{\text {off }}$ to the input and to close a feedback loop in time intervals between integration. The integrator output is thereby forced to a zero output level, regardless which effect is causing $U_{\text {off }} U_{\text {comp }}$ is stored and kept constant during integration by a sample and hold circuit. Additionally, operational amplifiers with low $\mathrm{U}_{\text {off }}$ (typ. $0.5 \square \mathrm{V}$ ) and low $\mathrm{dU}_{\text {off }} / \mathrm{dT}$ (typ. $0.01 \square \mathrm{V} / \mathrm{K}$ ) have to be chosen. These low values are only obtained in chopper 
stabilized operational amplifiers. This type of automatically drift compensated integrator has been improved at TS, where a symmetric variant could improve the drift to typical values of $0.135 \mathrm{mVs}$ after $1000 \mathrm{~s}$ [303]. The drift compensation of pure analogue integrators does not work perfectly, if the input signal is not quiescent. Although the integrator and the sample and hold circuit perform an averaging of the input signal, the overall circuit is typically equivalent to a low pass filter of first order. However, in devices with superconducting magnets, the magnetic field fluctuations caused by the current ripple of the power supply is usually rather low and such integrators are well suited for long pulse operation if the design of the circuit board is made properly and well selected electronic components are used.

The drift compensation can be improved with respect to noisy input signals by a digital circuit version of the drift compensation (Figure 13b). During the compensation phase the output signal is AD-converted allowing for the application of digital signal processing. Hence, any filter type of high order can be realized easily and $U_{\text {comp }}$ can be derived in this manner. The input amplifier is fed by the DA-converter of this stage [320] holding the appropriate offset voltage level during integration.

LHD has developed an analogue integrator concept by employing three analogue integrators simultaneously $[321,322]$. Each time interval is divided into three overlapping sub-intervals, in which the integrators perform the integration subsequently (Figure 13c). By digital signal processing the whole integrated signal is recovered. The advantage of such a system is due to the transformation of the long pulse into short pulse integrations in order to perform drift corrections of the inactive integrators. Linear drift of each integrator during the short integration interval can be assumed and the dynamic range is increased. Thereby, this integrator could be attributed to both analogue and digital integration.

Pure digital integration is performed within the integrator concept based on voltage to frequency converters at JT-60 [323]. The input voltage is converted to a square wave signal, whose frequency has a very accurate linear dependence $(<0.002 \%$ deviation of the full scale). The integration is performed simply by counting the frequency and to add them cumulative to a register (Figure 13d).

Another approach for digital integration including a dedicated offset correction has been developed at DIII-D [324]. The pick up coil signal is AD-converted and digitally integrated in a signal processing unit just by summing up the sample values. In order to get a measure of the drift, an electronic switch to dummy resistor has been added. During the measurement, the input is switched periodically to the resistor and the sample values are added to another register (Figure 13e). Thus, two different registers exist with integrated values of the offset alone and the sum of the induced and offset voltages. The drift could be reduced in this way by one order of magnitude.

For W7-X a somewhat different approach is planned. Usually, the quality of the integrator circuit depends on the quality of the input stage. In case of the classical analogue integrator and derived circuits (Figure 13a-d) it is the differential amplifier at the input stage which defines the overall quality most. Since active elements are usually semiconductors, the first element in the chain should be a switch, because any temperature dependence of non-linear current-voltage functions then becomes irrelevant in case the semiconductor is purely conducting or purely isolating. A further criterion is to make the raw induction signal as robust as possible. This can be achieved by chopping the polarity of the signal using fast analogue CMOS switches (Figure 13e). Once the signal is chopped, it is no longer vulnerable to thermoelectric and other nuisance voltages. Furthermore, it is beneficial to have the 
switching frequency at the lowest relevant frequency for the remaining signal chain. A high pass filter can be applied and the 1/f noise problem [318] could be mitigated in this way. An integrator prototype has been recently operated under real conditions at the WEGA stellarator [325].

This concept could be of advantage for burning plasma experiments, if the chopper is made of simple contacts like in standard relays and placed directly inside the plasma vessel as close as possible to the pick-up coils and the flux loops. All thermo-voltages and contact potential variations of the signal cables and feedthroughs are then no longer of concern.

All integrators have to be improved with respect to the common mode rejection. Such signals are introduced in machines with high neutron fluxes due to RIEMF and RIC (see chapter 11). However, even at devices with low neutron fluxes, common mode rejection could be of importance, if diagnostic coils design is based on MI-cables. The conductivity of the insulation material $(\mathrm{MgO})$ in these cables increases with temperature. Thus, common mode signals may appear if the integrator electronics and coils mounted on the plasmas vessel are at different electrostatic potentials.

\section{4}

\section{Alternative concepts}

The magnetic signal integration problem may be considered as solved on the basis of discharge durations in the order of one hour. However, real steady state plasmas in a reactor may have even higher demands on the long-term stability of the signals. It is worth to look on diagnostics, which deliver information on the magnetic field properties and which do not require any signal integration in time. Certainly there are physically caused limitations in finding a substitute for loop and coil signal measurements.

The possibilities are given for local magnetic flux density measurements outside the plasma. Many physical effects can be exploited, such like nuclear magnetic resonance measurements (NMR), hall sensors and even sensors, which exploit jxB forces. NMR measurements are well suited for the determination of the magnetic field strength, because the signal does not depend on the field direction. Recently, such a system has been used to re-investigate the vacuum magnetic field structure of W7-AS. It has the disadvantage, that it can be used in regions with low magnetic field gradients only. Hall-sensors have advantages due to their small size, since not much space is available for conventional pick-up coils in future long pulse devices [326]. However, these devices cannot be used at elevated temperatures and basically the output voltage is rather low. Sensors with integrated amplifiers are available [327] leading to useful voltage levels $(7 \mathrm{~V} / \mathrm{T}$ at $\mathrm{f}=0-20 \mathrm{kHz})$. As any semiconductor device, neutron bombardment in BPX may damage such devices. A combination of conventional inductive sensors and $j x B$ force measurements has been suggested for steady state measurements [328, 329]. In this system, the integrator drift is corrected by application of a current pulse into the pick-up coils exerting forces onto a strain gauge. This force depends on the absolute field strength allowing for the periodically correction of the integrated signal. A similar method, where a pick-up coil measurement is long term stabilized by mechanical motion, is based on a rotating pick-up coil [330].

Inside the plasma, atomic line spectra and the polarization degree of the emitted light carry information at least on the field direction as exploited in the motional Stark effect [331] and also for the plasma edge by the Li-beam diagnostic [332]. In particular for the tokamak this 
system is very helpful to determine the q-profile, which otherwise could be only determined if the current profile is known.

The measurement of the flux density along a given contour can be performed by virtue of the Faraday-rotation. Inside the plasma, the contour is typically a line of sight which measures the integral [333]. Outside the plasma, this effect can be exploited to replace the Rogowski-coil [334]. In this case, an optical fibre has to follow a contour enclosing the current. A precondition on the fibre is to keep the polarization state of the input light in order to avoid phase changes on the left and right circularly polarized by the stresses birefringence or the bending of the fibre itself. Such systems are already in use for current measurements in electrical power grids but only available for a circular contour.

Alternative diagnostic methods for flux measurements are hardly conceivable. A general alternative using flux loops would be to change the area normal in time, like a rotating coil delivering a periodic signal with the amplitude being directly proportional to the flux. Of course, for large loops like voltage and flux loops it is impossible to make such an area variation and for the diamagnetic measurements it would intersect the plasma. A second method could be to transfer the flux out of the plasma by superconductors, by enclosing the plasma with a loop, connecting it to a superconducting bus system and to let the induced current flow through an external loop. Inside the external loop, a rotating flux loop could be easily installed. A third approach would be to find a measure for the vector potential along a given contour. Since a direct measure of the vector potential is not possible, only quantum mechanical phase changes could be exploited [335]. The measurements of quantum mechanical interferences, however, can hardly be imagined on such large dimensions, like the poloidal circumference due to the short de Broglie-wavelength involved.

\subsection{Automated analysis}

In a long pulse experiment the automated analysis of magnetic data is essential for the evaluation of almost all discharge data. In short pulse experiments, a comprehensive analysis has typically been performed manually, mostly for a subset of discharges. In particular information derived from magnetic diagnostics, the equilibrium reconstruction and the spatiotemporal analysis of MHD activity, is a challenge for automated analysis. The equilibrium information, on the other hand, is key information for most of the other diagnostics and the automated mode analysis of fluctuations can become an issue for safe machine operation, e.g. early detection of locked modes for disruption mitigation.

For tokamaks, the automated equilibrium reconstruction is well developed. This is natural, since the equilibrium strongly depends on the toroidal current profiles and without such tools no estimates on flux surfaces are possible. Elongated tokamak plasmas have to be stabilized actively and the plasma shape reconstruction is essential for the avoidance of first wall element overloading and the divertor control. A comparison of different reconstruction methods with respect to the plasma shape accuracy has been performed for ITER [23] comprising artificial neural networks, function parameterisation and EFIT. Dedicated fast algorithms for plasma shape determination are reported for tokamaks based on a filament representation of the plasma current, in particular for plasma control and stabilization [336], but also EFIT [337] has been used for shape control at DIII-D [338]. The development of JET plasma shape control system and equilibrium reconstruction, using XLOC, XSC, EQUINOX and EFIT, is reported in [22]. 
The stellarator equilibrium reconstruction has to be performed in 3 dimensions and, hence, more effort is needed to obtain equilibrium information on demand. The free boundary VMEC code [339] is able to calculate the equilibrium surfaces on a few minutes to hour time scale, depending on the required accuracy and host system. This code assumes nested flux surfaces and is, hence, not suited to model boundary island configurations and internal island chains. At present, two codes are in use which do not rely on flux surfaces, namely HINT [340] and PIES [341]. These codes are able to describe the MHD equilibrium including islands and ergodic structures, but the computational effort is very large. At present, all the 3D MHD equilibrium codes are far away from being real-time capable. Thus, a database for the storage of such (expensive) equilibrium calculations is used for W7-X, which will allow to select pre-computed equilibria for a given input data set, like magnetic configuration, limiter setup and pressure and current profiles [342].

Tools for fast equilibrium reconstruction have been developed in the past, which are based on such pre-computed stellarator equilibria employing the free boundary VMEC version. Beside pre-computed equilibria, efficient methods for calculating the theoretically expected coil signals are needed (DIAGNO[343], V3RFUN [344]). These calculations for equilibrium and coil signal may serve as training set for artificial neural networks or as the basis for the function parameterisation $[345,346]$. The function parametrization and an artificial neural network are under development for $\mathrm{W} 7-\mathrm{X}$ and comprise so far vacuum magnetic field configurations, which include the separatrix position and the width of internal island chains in non-standard configurations [347]. These tools will enable real-time stellarator equilibrium reconstruction at least for those configurations, which are already known and contained in the training set. Once a fast and validated method for the equilibrium reconstruction has been established, the integrated data analysis approach becomes possible. It has been demonstrated for W7-AS, that even the position and shape uncertainty of flux surfaces can be deduced by incorporating many diagnostic systems via Bayesian graphical models [98].

Beside the challenges in determining the equilibrium sufficiently fast, the automated mode analysis is essential for a routine and systematic evaluation of the observed MHD activities of the plasma. This leads to a new quality in comparing theoretical models describing such instabilities with experimental data of all discharges. The frequency determination and phase correlation between different signals of coils at different positions can be done straight forward by well-known tools, like the fast Fourier transform or wavelet analysis. It is more difficult to obtain information on the angular spatial information of the plasma oscillation, in particular when the flux surface geometry is strongly oscillating like in poloidal direction in elongated tokamaks [348] or in stellarator configurations. The poloidal and toroidal mode numbers are mandatory for a reasonable comparison with theory, because the frequency of the mode may be modified by plasma velocities. Since the phase of single coil signals is affected by the flux surface oscillation, it is essential to correct all other phase errors. These can be caused by resonances in the coil-cable system and screening currents in the surrounding material [317]. Beside well-known methods for mode number identification like cross correlation and zero transition counting techniques, the mode identification can be enhanced by applying feature extraction methods, like singular value decomposition. Recently, a method for mode identification using unevenly spaced probes (in the frame of flux surface coordinates) has been developed, which gives probabilities only for the occurrence of mode numbers [349]. It combines the features of a wavelet transform and the Lomb-periodogram and is intended to be used in the integrated data analysis framework. 


\section{Summary and Conclusions}

The diagnostic systems of an increasing number of fusion experiments dedicated to long pulse high power non-BPX operation is facing a variety of problems reviewed in this paper. Most severe problems are connected with the thermal loading of the front-end components i.e. windows and mirrors of the optical diagnostics by UV- und VUV-radiation from the plasma, which demands for their active cooling. All plasma facing optical components are being degraded in addition by surface modifications and erosion due to the bombardment by energetic particles resulting from charge exchange processes in the plasma.

Suitable design solutions for actively cooled high heat load plasma facing vacuum windows, which are compatible with the thermo-mechanical properties of the various materials covering the spectral range from $110 \mathrm{~nm}$ to $\sim 10 \mu \mathrm{m}$, still need to be demonstrated for diameters in excess of a few centimetres. Various promising ideas exist and are presently being investigated in high heat load and ECRH stray radiation test chambers. As an interim solution for not too high power loads and pulse lengths an un-cooled window plate in front of the vacuum window could be foreseen before going to fully developed mirror-based systems as planned for KSTAR. Protection of plasma facing vacuum windows and/or the interior materials inside diagnostic tubes from ECRH stray radiation by micromeshes might prove unavoidable in same cases.

A point of major concern in long pulse experiments with carbon divertors is the build-up of predominantly soft a-C:H layers, in particular on wide angle observations systems which need to be located in the vicinity of the plasma wall, which will lead to intolerably high window transmission losses. A few days of long pulse operation easily corresponds one year of operation on present day pulsed fusion experiments. As recent in-situ measurements of contamination build-up suggest, keeping window protecting shutters closed whenever possible during the first two discharges of a day and in-between pulses might significantly improve the situation, however, the developments of active cleaning techniques either by thermal annealing, i.e. by heating windows, which need to be cooled during the experiments, to about $400{ }^{\circ} \mathrm{C}$ over night and during weekends, by photo-cleaning or even by plasma etching is essential. This needs to be accompanied by integration of in-situ calibration and possibly contamination build-up monitoring systems in the diagnostic designs.

While in non-BPX experiments n- and G-radiation do not have any detectable effect on windows, in optical fibres induced luminescence might have to be corrected for in high power experiments. Radiation induced absorption in optical fibres usually is insignificant, with sufficiently radiation hard fibres being commercially available for non-BPX for the spectral region from about $300 \mathrm{~nm}$ into the IR region. Even for the ITER environment significant progress has been made in the development of radiation hardened fibres in recent years, with the reduction of fibre luminescence being the main remaining, though not optical measurements preventing, challenge. For the spectral region below $350 \mathrm{~nm}$ no satisfying solution has been found yet.

In burning plasma experiments only mirrors can be used as first plasma facing optical component, however, also in non-BPX devices the use of mirrors can have significant advantages. They are easier to cool, contamination by deposits usually results in lesser reflectivity losses than transmission losses by coatings on windows and e.g. single crystalline $\mathrm{W}$ or Mo mirrors or $\mathrm{Rh}$ on polycrystalline $\mathrm{Cu}$ are believed to have sufficient live-times when exposed to erosion by CX neutrals even in ITER. All these effects can be reduced to insignificant levels by use of entrance apertures in front of the first mirror. Experiments with exposition of various mirror types are ongoing on all major fusion devices and are regarded as a high priory issue in ITER diagnostic development.

The other major concern is connected with the magnetic diagnostics in particular with the long-time electronic integrators used with inductance loops. However, due to recent progress 
in this field the conventional magnetic diagnostics are now well developed for use in long pulse experiments with pulse durations in the order of $1000 \mathrm{~s}$, even applicable in stellarators with strongly reduced internal currents. Concepts for the electronic design of the signal integrators have been successfully developed and used in the past, but care has to be taken for contact and thermo-electric voltages in the signal path.

Additional challenges will occur in the first burning plasma experiments conducted in ITER. Here, the major effects are the radiation induced currents and conductivity, which lead to a common mode signal disturbing the signal integration of inductive sensors. Alternative concepts for external measurements of magnetic flux densities look promising, but no real alternative for flux measurements exists. The diagnostic integration into the first wall elements is usually a complex task, comprising the trade-off in signal perturbation by screening currents and a proper thermal design. Although future experiments grow in size, not much space is left for diagnostics due to thermal shields and blanket modules. In particular the magnetic fluctuation diagnostics needs inductive sensors being close to the plasma edge and being able to detect signal frequencies up to $1 \mathrm{MHz}$. The required automated magnetic equilibrium analysis is well developed for tokamaks, even for real-time applications. For stellarators, conceptual designs exist on the basis of function parametrization, artificial neural networks and well-structured databases. Routinely use, however, has still to be demonstrated.

To prepare diagnostic components for long pulse operation is primarily a technical challenge, ranging from the window and mirror requirements of the optical plasma diagnostics and the integrator problem to the development of reliable diagnostics for the careful monitoring of all the other actively cooled plasma facing components. Successful operation has already been demonstrated in Tore Supra. It is obvious in spite of still open questions, mainly connected with the harsh environment of future burning plasma experiments, that the combined effort of various programs in a number of institutions around the world will enable the generation of steady state fusion experiments to parry the challenge. 


\section{References}

[1] Costley, A.E., T. Sugie, G. Vayakis et al., Technological challenges of ITER diagnostics. Fusion Engineering and Design, 2005. 74(1-4): p. 109-119.

[2] ITER, Progress in the ITER Physics Basis. Special issue of Nucl. Fusion, to appear, 2006. chapter 7, Diagnostic.

[3] Donne, A.J.H. and A.E. Costley, Key issues in diagnostics for burning plasma experiments. IEEE Transactions on Plasma Science, 2004. 32(1): p. 177-186.

[4] van Houtte, D., G. Martin, A. Becoulet et al., Recent fully non-inductive operation results in Tore Supra with 6 min, 1 GJ plasma discharges. Nuclear Fusion, 2004. 44(5): p. L11-L15.

[5] J. Jaquinot on behalf of the Tore Supra Team. Steady-State Operation of Tokamaks : Key Physics and technology Developments on Tore Supra. in Proc. 20th Int. Conf. on Fusion Energy. 2004. Vilamoura, IAEA, Wien Conference Proceedings CD-ROM file $\mathrm{OV} / 2-2$,

[6] Ide, S., t.J.-. Team, T. Suzuki et al. Overview of JT-60U Progress towards Steadystate Advanced Tokamak. in Proc. 20th Int. Conf. on Fusion Energy. 2004. Vilamoura, IAEA, Wien Conference Proceedings CD-ROM file OV/1-1,

[7] Zushi, H., K. Nakamura, K. Hanada et al. Overview of stedy-state tokamak operation and current drive experiments in TRIAM-1M. in Proc. 20th Int. Conf. on Fusion Energy. 2004. Vilamoura, IAEA, Wien Conference Proceedings CD-ROM file OV/52 ,

[8] Kumazawa, R., T. Mutoh, K. Saito et al., Long-pulse plasma discharge on the Large Helical Device. Nuclear Fusion, 2006(3): p. S13-S21.

[9] Yamamoto, S., L. De Kock, V. Belyakov et al., Irradiation Tests on ITER Diagnostic Components, in International School of Plasma Physics "Piero Caldirola" Workshop on Diagnostics for Experimental Fusion Reactors 2, ed. P.E. Stott, G. Gorini, P. Prandoni, and E. Sandoni, 1998, New York and London: Plenum Press, p. 269.

[10] Ibarra, A. and E.R. Hodgson, The ITER project: the role of insulators. Nuclear Instruments \& Methods in Physics Research Section B-Beam Interactions with Materials and Atoms, 2004. 218: p. 29-35.

[11] Decreton, M., T. Shikama, and E. Hodgson, Performance of functional materials and components in a fusion reactor: the issue of radiation effects in ceramics and glass materials for diagnostics. Journal of Nuclear Materials, 2004. 329-33: p. 125-132.

[12] Barabash, V., G. Federici, J. Linke et al., Material/plasma surface interaction issues following neutron damage. Journal of Nuclear Materials, 2003: p. 42-51. 
[13] Hodgson, E.R., Radiation Problems for Insulators in ITER and Beyond. Problems of Atomic Science and Technology, Series: Plasma Physics 2002. 4(7): p. 76.

[14] Young, K.M., A.E. Costley, R. Bartiromo et al., Chapter 7: Measurement of plasma parameters. Nuclear Fusion, 1999. 39(12): p. 2541-2575.

[15] Costley, A., K. Ebisawa, P. Edmonds et al., Overview of the ITER Diagnostic System, in International School of Plasma Physics "Piero Caldirola" Workshop on Diagnostics for Experimental Fusion Reactors 2, ed. P.E. Stott, G. Gorini, P. Prandoni, and E. Sandoni, 1998, New York and London: Plenum Press, p. 41.

[16] Costley, A., Requirements and Issues in Diagnostics for Next Step Burning Plasma Experiments, in Advanced Diagnostics for Magnetic and Inertial Fusion, ed. P.E. Stott, A. Wotton, G. Gorini, E. Sindoni, and D. Batani, 2002, New York: Kluwer Academic/Plenum Publishers, p. 1.

[17] Aymar, R., ITER status, design and material objectives. Journal of Nuclear Materials, 2002. 307: p. 1-9.

[18] Lister, J.B., P.L. Bruzzone, A.E. Costley et al., Technical issues associated with the control of steady state tokamaks. Nuclear Fusion, 2000. 40(6): p. 1167-1181.

[19] Turkin, Y.A., H. Maassberg, C.G. Beidler et al. Predictive Transport Modeling for W7-X. in 31st EPS Conference on Plasma Physics. 2004. London UK, Conference Proceedings ECA Vol. 28G, P-1.198

[20] Fischer, R., A. Dinklage, and E. Pasch, Bayesian modelling of fusion diagnostics. Plasma Physics and Controlled Fusion, 2003. 45(7): p. 1095-1111.

[21] Hennig, C., P. Heimann, S. Heinzel et al., A concept of online monitoring for the Wendelstein 7-X experiment. Fusion Engineering and Design, 2004. 71(1-4): p. 107110.

[22] Murari, A., E. Joffrin, R. Felton et al., Development of real-time diagnostics and feedback algorithms for JET in view of the next step. Plasma Physics and Controlled Fusion, 2005. 47(3): p. 395-407.

[23] de Kock, L., C. Walker, E. Coccorese et al., Design of the magnetic diagnostic for ITER. Review of Scientific Instruments, 1999. 70(1): p. 452.

[24] Laviron, C., Strategy of the Tore Supra Diagnostic Set for Steady State High Power Operation, in Advanced Diagnostics for Magnetic and Inertial Fusion, ed. P.E. Stott, A. Wotton, G. Gorini, E. Sindoni, and D. Batani, 2002, New York: Kluwer Academic/Plenum Publishers, p. 87.

[25] Mitarai, O. and K. Muraoka, Ignition Burn Control and Diagnostics in ITER. 2 ed. Diagnostics for Experimental Thermonuclear Fusion Reactors ed. G.G. Peter Stott, Paolo Prandoni and Elio Sindoni. 1998, New York and London: Plenum Press p. 93.

[26] Kusama, Y., Requirements for Diagnostics in Controlling Advanced Tokamak Modes in Advanced Diagnostics for Magnetic and Inertial Fusion, ed. P.E. Stott, A. Wotton, 
G. Gorini, E. Sindoni, and D. Batani, 2002, New York: Kluwer Academic/Plenum Publishers, p. 31.

[27] Sips, A.C.C. and I.T.P. Activity, Advanced scenarios for ITER operation. Plasma Physics and Controlled Fusion, 2005. 47: p. A19-A40.

[28] Saoutic, B., Status of long pulse experiments in magnetic fusion devices. Plasma Physics and Controlled Fusion, 2002(12B): p. B11-B22.

[29] Guillerminet, B., Y. Buravand, M. Le Luyer et al., Tore Supra continuous acquisition system. Fusion Engineering and Design, 2002. 60(3): p. 427-434.

[30] Saint Laurent, F., J. Bucalossi, and G. Martin, Real Time Control for Steady State Operation at Tore Supra. IAEA-TCM on Steady State operation of Magnetic Fusion Devices, 2002, Arles, France.

[31] Giruzzi, G., F. Imbeaux, J.L. Segui et al., New tokamak plasma regime with stationary temperature oscillations. Physical Review Letters, 2003. 91(13): p. 135001.

[32] Raupp, G., G. Neu, W. Treutterer et al., Control process structure of ASDEX Upgrade's new Control and Data Acquisition System. Fusion Engineering and Design, 2005. 74(1-4): p. 697-705.

[33] Sips, A., J. Hobirk, and A.G. Peeters, Chapter 4: Advanced tokamak studies in ASDEX Upgrade. Fusion Science and Technology, 2003. 44(3): p. 605-617.

[34] Kallenbach, A., P.T. Lang, R. Dux et al., Integrated exhaust control with divertor parameter feedback and pellet ELM pacemaking in ASDEX Upgrade. Journal of Nuclear Materials, 2005. 337-39(1-3): p. 732-736.

[35] Burrell, K.H., Diagnostics for advanced tokamak research (invited). Review of Scientific Instruments, 2001. 72(1): p. 906-914.

[36] Colas, L., E. Faudot, S. Bremond et al. Theory and Practice in ICRF Antennas for Long Pulse Operation. in RADIO FREQUENCY POWER IN PLASMAS: 16th Topical Conference on Radio Frequency Power in Plasmas. 2005. Park City, Utah (USA), AIP Conference Proceedings 787, 150-157

[37] Martin, G. and Tore Supra Team, Overview of Steady-State Operation on the Tore Supra Tokamak. IAEA-TCM on Steady State operation of Magnetic Fusion Devices, 2002, Arles, France.

[38] Moreau, P., J. Bucalossi, C. Brosset et al. Long Pulse Operation on Tore-Supra: Towards Steady State. in PLASMA 2005: Int. Conf. on Research and Applications of Plasmas; 3rd German-Polish Conf.on Plasma Diagnostics for Fusion and Applications; 5th French-Polish Seminar on Thermal Plasma in Space and Laboratory. 2006. Opole-Turawa (Poland), AIP Conference Proceedings 812, 11-18

[39] Seki, T., R. Kumazawa, T. Mutoh et al., Steady-state amplifier at megawatt level for LHD ICRF heating. Fusion Science and Technology, 2001. 40(3): p. 253-64. 
[40] Sudo, S., B.J. Peterson, K. Kawahata et al., LHD diagnostics toward steady-state operation. IEEE Transactions on Plasma Science, 2004. 32(1): p. 167-176.

[41] Jernigan, T.C., T.S. Bigelow, R.J. Colchin et al., Long-Pulse Experiments on the Advanced Toroidal Facility. Physics of Plasmas, 1995. 2(6): p. 2435-2439.

[42] Lyon, J.F., B.A. Carreras, K.K. Chipley et al., The Advanced Toroidal Facility. Fusion Technology, 1986. 10(2): p. 179-226.

[43] Renner, H., J. Boscary, V. Erckmann et al., The capabilities of steady state operation at the stellarator W7-X with emphasis on divertor design. Nuclear Fusion, 2000. 40(6): p. 1083-1093.

[44] Renner, H., J. Boscary, H. Greuner et al., Divertor concept for the W7-X stellarator and mode of operation. Plasma Physics and Controlled Fusion, 2002. 44(6): p. 10051019.

[45] Reichle, R., D. Guilhem, R. Mitteau et al., Status of power balance monitoring for long pulse operation at Tore Supra. Nuclear Fusion, 2003. 43(9): p. 797-804.

[46] Guilhem, D., G. Martin, R. Reichle et al., Infrared surface temperature measurement for long pulse real time feed-back control in an actively cooled machine. Review of Scientific Instruments, 1999. 70(1): p. 427-430.

[47] Bucalossi, J. and Tore Supra Team, Problems and issues for tokamak long-pulse operation: Major progress on Tore Supra. Fusion Science and Technology, 2004. 46(1): p. 184-191.

[48] Grisolia, C. and Tore Supra Team, Plasma wall interaction during long pulse operation in Tore Supra. Journal of Nuclear Materials, 1999. 269: p. 146-152.

[49] Seki, T., T. Mutoh, R. Kumazawa et al. Long Pulse Plasma Heating Experiment by Ion Cyclotron Heating in LHD. in RADIO FREQUENCY POWER IN PLASMAS: 16th Topical Conference on Radio Frequency Power in Plasmas. 2005. Park City, Utah (USA), AIP Conference Proceedings 787, 98-105

[50] Costley, A., R. Bartiromo, L. De Kock et al., Requirements for ITER Diagnostics. Diagnostics for Experimental Thermonuclear Fusion Reactors, ed. P.E. Stott, G. Gorini, and E. Sindoni. 1996, New York and London: Plenum Press. p. 1.

[51] Hartfuss, H.J., R. Brakel, M. Endler et al., Diagnostic strategy of the W7-X stellarator. Review of Scientific Instruments, 1997. 68(2): p. 1244-1249.

[52] Kick, M., H.J. Hartfuss, J.V. Hofmann et al., Overview of W7-X Diagnostics. Fusion Engineering and Design, 1997. 34-5: p. 817-821.

[53] Grieger, G., W. Lotz, P. Merkel et al., Physics Optimization of Stellarators. Physics of Fluids B-Plasma Physics, 1992. 4(7): p. 2081-2091. 
[54] Hofmann, J.V., H.J. Hartfuss, H. Ringler et al., Overview of W7-X Diagnostics. Diagnostics for Experimental Thermonuclear Fusion Reactors, ed. P.E. Stott, G. Gorini, and E. Sindoni. 1996, New York and London: Plenum Press. p. 607.

[55] Sudo, S., H.J. Hartfuss, T. Mizuucchi et al., Diagnostics for Helical Systems. Advanced Diagnostics for Magnetic and Inertial Fusion, ed. P.E. Stott, A. Wotton, G. Gorini, E. Sindoni, and D. Batani. 2002, New York: Kluwer Academic/Plenum Publishers. p. 363.

[56] Ebisawa, K., A.E. Costley, A.J.H. Donne et al., Plasma diagnostics for INTER-FEAT. Review of Scientific Instruments, 2001. 72(1): p. 545-550.

[57] Nishitani, T., T. Shikama, M. Fukao et al., Neutron irradiation tests on diagnostic components at JAERI. Fusion Engineering and Design, 2001. 56-57: p. 905-909.

[58] Takatsu, H., K. Sato, K. Hamada et al., Overview on materials R\&D activities in Japan towards ITER construction and operation. Journal of Nuclear Materials, 2004. 329-333(Part 1): p. 178-182.

[59] Donne, A.J.H. and et al. Progress with High Priority R\&D Topics in Support of ITER/BPX Diagnostic Development. in 20th IAEA Fusion Energy Conference. 2004. Vilamoura, Portugal, Conference Proceedings, IT/P3-19

[60] Voitsenya, V., A.E. Costley, V. Bandourko et al., Diagnostic first mirrors for burning plasma experiments (invited). Review of Scientific Instruments, 2001. 72(1): p. 475482.

[61] Voitsenya, V.S., On the mechanism of contamination of diagnostic windows in fusion devices. Review of Scientific Instruments, 1999. 70(1): p. 787-789.

[62] Balden, M., A.F. Bardamid, A.I. Belyaeva et al., Surface roughening and grain orientation dependence of the erosion of polycrystalline stainless steel by hydrogen irradiation. Journal of Nuclear Materials, 2004. 329-33: p. 1515-1519.

[63] Gott, Y.V., Radiation effects on the components of diagnostic systems for a thermonuclear reactor: A review. Instruments and Experimental Techniques, 2005. 48(4): p. 423-432.

[64] Noda, N., Y. Nakamura, Y. Takeiri et al., Experimental studies towards long pulse steady state operation in LHD. Nuclear Fusion, 2001. 41(6): p. 779-790.

[65] Morita, S. and M. Goto, Space-resolved VUV spectroscopy using the $3 \mathrm{~m}$ normal incidence spectrometer with back-illuminated CCD detector in the LHD. Review of Scientific Instruments, 2003. 74(3): p. 2036-2039.

[66] Nagayama, Y., K. Kawahata, A. England et al., Electron cyclotron emission diagnostics on the large helical device. Review of Scientific Instruments, 1999. 70(1): p. 1021-1024. 
[67] Ozaki, T., S. Murakami, and P. Goncharov, Spatial resolved high-energy particle diagnostic system using time-of-flight neutral particle analyzer in Large Helical Device. Review of Scientific Instruments, 2003. 74(3): p. 1878-1882.

[68] Liang, Y., K. Ida, S. Kado et al., Photon-counting CCD detector as a tool of $x$-ray imaging. Review of Scientific Instruments, 2001. 72(1): p. 717-720.

[69] Peterson, B.J., A.Y. Kostrioukov, N. Ashikawa et al., Bolometer diagnostics for oneand two-dimensional measurements of radiated power on the Large Helical Device. Plasma Physics and Controlled Fusion, 2003. 45(7): p. 1167-1182.

[70] Gasparino, U., V. Erckmann, H.J. Hartfuss et al., Transport analysis through heat waves driven at different radial positions. Plasma Physics and Controlled Fusion, 1998. 40(2): p. 233-244.

[71] Hartfuss, H.J., M. Hase, C. Watts et al., Temperature fluctuation measurements with ECE on W7-AS. Plasma Physics and Controlled Fusion, 1996. 38(12A): p. A227A236.

[72] Watts, C., H.J. Hartfuss, and M. Hase, Comparison of different methods of electron cyclotron emission-correlation radiometry for the measurement of temperature fluctuations in the plasma core. Review of Scientific Instruments, 2004. 75(10): p. 3177-3184.

[73] Fredian, T.W. and J.A. Stillerman, MDSplus. Current developments and future directions. Fusion Engineering and Design, 2002. 60(3): p. 229-233.

[74] Hideya, N., K. Mamoru, O. Masaki et al., Steady-state data acquisition method for LHD diagnostics. Fusion Engineering and Design, 2003. 66-8: p. 827-832.

[75] Luo, J.R., L. Zhu, H.Z. Wang et al., Towards steady-state operational design for the data and PF control systems of the HT-7U. Nuclear Fusion, 2003. 43(9): p. 862-869.

[76] Heimann, P., S. Heinzel, C. Hennig et al., Status report on the development of the data acquisition system of Wendelstein 7-X. Fusion Engineering and Design, 2004. 71(1-4): p. 219-224.

[77] Schacht, J., H. Niedermeyer, C. Wiencke et al., A trigger-time-event system for the W7-X experiment. Fusion Engineering and Design, 2002. 60(3): p. 373-379.

[78] Reetz, J., P. Heimann, S. Heinzel et al., Image data acquisition in the scope of long duration discharges. Fusion Engineering and Design, 2004. 71(1-4): p. 231-237.

[79] How, J.A., J.W. Farthing, and V. Schmidt, Trends in computing systems for large fusion experiments. Fusion Engineering and Design, 2004. 70(2): p. 115-122.

[80] Erckmann, V., P. Brand, H. Braune et al. The $10 \mathrm{MW}, \mathrm{CW}$ ECRH System For W7-X: Status And First Integrated Tests. in RADIO FREQUENCY POWER IN PLASMAS: 16th Topical Conference on Radio Frequency Power in Plasmas. 2005. Park City, Utah (USA), AIP Conference Proceedings 787, 371-378 
[81] Nowak, S. and A. Orefice, 3-Dimensional Propagation and Absorption of HighFrequency Gaussian Beams in Magnetoactive Plasmas. Physics of Plasmas, 1994. 1(5): p. 1242-1250.

[82] Gandini, F., M. Hirsch, S. Cirant et al. Measurement of stray EC radiation on W7-AS. in RADIO FREQUENCY POWER IN PLASMAS:14th Topical Conference. 2001. Oxnard,California (USA), AIP Conference Proceedings 595, 358-361

[83] Laqua, H.P. and et al. Distribution of the ECRH stray radiation in fusion devices. in 28th Conference on Control. Fusion and Plasma Phys. 2001. Funchal , Madeira, Conference Proceedings ECA 25A, 1277

[84] Nowak, S., A. Airoldi, A. Bruschi et al., Measurements of residual power of EC waves propagating in FTU tokamak during ECRH experiments at $140 \mathrm{GHz}$. Fusion Engineering and Design, 2001. 53: p. 315-320.

[85] Prater, R., Heating and current drive by electron cyclotron waves. Physics of Plasmas, 2004. 11(5): p. 2349-2376.

[86] Erckmann, V. and U. Gasparino, Electron-Cyclotron-Resonance Heating and Current Drive in Toroidal Fusion Plasmas. Plasma Physics and Controlled Fusion, 1994. 36(12): p. 1869-1962.

[87] Laqua, H.P., V. Erckmann, H.J. Hartfuss et al., Resonant and nonresonant electron cyclotron heating at densities above the plasma cutoff by $O-X-B$ mode conversion at the W7-AS stellarator. Physical Review Letters, 1997. 78(18): p. 3467-3470.

[88] Gandini, F., S. Cirant, M. Hirsch et al., The detection of the non-absorbed millimeterwave power during EC heating and current drive. Fusion Engineering and Design, 2001. 56-7: p. 975-979.

[89] Sanchez, M. and J. Sanchez, Thermo-optical effect in zinc selenide windows for twocolor interferometer for fusion plasma diagnostics. Review of Scientific Instruments, 2005. 76(4): p. 046104-4.

[90] Lamb, J.W., Miscellaneous data on materials for millimetre and submillimetre optics. International Journal of Infrared and Millimeter Waves, 1996. 17(12): p. 1997-2034.

[91] Simonis, G.J., Index to the literature dealing with the near-millimeter wave properties of materials. International Journal of Infrared and Millimeter Waves, 1982. 3(4): p. 439-469.

[92] Ullrich, S., Charakterisierung einer Materialtestkammer als MillimeterwellenResonator bei $140 \mathrm{GHz}, 2005$, Diploma Thesis, Ernst-Moritz-Arndt-University, Greifswald, Germany.

[93] Hartfuss, H.J. and et al. Problems with ECRH stray radiation in Wendelstein 7-X. in Proc of 30th Conference on Contr. Fusion and Plasma Phys. 2003. St. Petersburg, Russia, Conference Proceedings ECA Vol. 27A, O-3.2C 
[94] Laqua, H., The launching scheme is based on an idea of H. Laqua of Max-Planck Institut für Plasmaphysik, Greifswald, Germany.

[95] Wanner, M., V. Erckmann, J.H. Feist et al., Status of WENDELSTEIN 7-X construction. Nuclear Fusion, 2003. 43(6): p. 416-424.

[96] Walker, C.I., A.E. Costley, K. Itami et al., ITER diagnostics: Integration and engineering aspects. Review of Scientific Instruments, 2004. 75(10): p. 4243-4246.

[97] Fischer, R. and A. Dinklage, Integrated data analysis of fusion diagnostics by means of the Bayesian probability theory. Review of Scientific Instruments, 2004. 75(10): p. 4237-4239.

[98] Svensson, J., A. Dinklage, J. Geiger et al., Integrating diagnostic data analysis for W7-AS using Bayesian graphical models. Review of Scientific Instruments, 2004. 75(10): p. 4219-4221.

[99] Dinklage, A., R. Fischer, and J. Svensson, Topics and methods for data validation by means of Bayesian probability theory. Fusion Science and Technology, 2004. 46(2): p. 355-364.

[100] Equipe, T.F.R., Nuclear Fusion, 1978. 18: p. 647.

[101] Stangeby, P.C., Effect of Bias on Trapping Probes and Bolometers for Tokamak Edge Diagnosis. Journal of Physics D-Applied Physics, 1982. 15(6): p. 1007-1029.

[102] Davies, S.J., X. Tellier, G.F. Matthews et al., Development of popup Langmuir probe system for the JET MkIIa divertor. Fusion Engineering and Design, 1999. 46(1): p. $27-$ 35.

[103] Pedrosa, M.A., A. Lopez-Sanchez, C. Hidalgo et al., Fast movable remotely controlled Langmuir probe system. Review of Scientific Instruments, 1999. 70(1): p. 415-418.

[104] Von Hellermann, M.G., W.G.F. Core, A. Howman et al., Feasibility of Quantitative Spectroscopy on ITER. Diagnostics for Experimental Thermonuclear Fusion Reactors, ed. P.E. Stott, G. Gorini, and E. Sindoni. 1996, New York and London: Plenum Press. p. 321.

[105] König, R., O. Ogorodnikova, D. Hildebrandt et al., Development of cooled UV, visible and IR windows for quasicontinuous operation of the W7-X stellarator. Review of Scientific Instruments, 2004. 75(10): p. 4258-4260.

[106] Hawkes, N.C., R. DeAngelis, S. Dillon et al., XUV and VUV Spectroscopy of ITER, in International School of Plasma Physics "Piero Caldirola" Workshop on Diagnostics for Experimental Fusion Reactors 2, ed. P.E. Stott, G. Gorini, P. Prandoni, and E. Sandoni, 1998, New York and London: Plenum Press, p. 297.

[107] Von Hellermann, M. and et al. in 30th EPS Conference on Controlled Fusion and Plasma Physics. 2003. St. Petersburg, Russia, Conference Proceedings ECA Vol. 27A, O-4.2D 
[108] Malaquais, A., M. Von Hellermann, P. Lotte et al. Polarization and Reflectivity Changes on Mirror Based Systems during long pulse Operation. in 30th EPS Conference on Controlled Fusion and Plasma Physics. 2003. St. Petersburg, Russia, Conference Proceedings ECA Vol. 27A, O-3.4C

[109] Herrmann, A., Optical Surface Temperature Measurement. Diagnostics for Experimental Thermonuclear Fusion Reactors, ed. P.E. Stott, G. Gorini, and E. Sindoni. 1996, New York and London: Plenum Press. p. 581.

[110] Hildebrandt, D., D. Naujoks, and D. Sunder, Surface temperature measurements of carbon materials in fusion devices. Journal of Nuclear Materials, 2005. 337-39(1-3): p. 1064-1068.

[111] Giannone, L., Experiments are being conducted at ASDEX Upgrade. Private Communication.

[112] Reichle, R., J.C. Fuchs, R.M. Giannella et al., Bolometer for ITER. Diagnostics for Experimental Thermonuclear Fusion Reactors, ed. P.E. Stott, G. Gorini, and E. Sindoni. 1996, New York and London: Plenum Press. p. 559.

[113] Wurden, G.A. and B.J. Peterson, Imaging Bolometry Development for Large Fusion Devices, in International School of Plasma Physics "Piero Caldirola" Workshop on Diagnostics for Experimental Fusion Reactors 2, ed. P.E. Stott, G. Gorini, P. Prandoni, and E. Sandoni, 1998, New York and London: Plenum Press, p. 399.

[114] Peterson, B.J., A.Y. Kostrioukov, N. Ashikawa et al., Calibration and sensitivity of the infrared imaging video bolometer. Review of Scientific Instruments, 2003. 74(3): p. 2040-2043.

[115] Peterson, B.J., N. Ashikawa, S. Konoshima et al. Imaging Bolometer for Burning Plasma Experiment. in 30th EPS Conference on Controlled Fusion and Plasma Physics. 2003. St. Petersburg, Conference Proceedings ECA Vol. 27A, P-4.067

[116] Konoshima, S., B.J. Peterson, N. Ashikawa et al. Radiated Power Profile Observed by Tangentially Viewing IR Bolometer in JT-60U Tokamak. in 32nd EPS Conference on Controlled Fusion and Plasma Physics. 2005. Tarragona, Spain, Conference Proceedings ECA Vol. 29C, P-4.092

[117] Gonzalez, M. and E.R. Hodgson, Radiation resistant alternative substrates for ITER bolometers. Fusion Engineering \& Design, 2003. 66-8: p. 881-885.

[118] Sheffield, J., Plasma Scattering of Electromagnetic Radiation. 1975, New York: Academic Press.

[119] Pasqualotto, R., P. Nielsen, C. Gowers et al., High resolution Thomson scattering for Joint European Torus (JET). Review of Scientific Instruments, 2004. 75(10): p. 38913893.

[120] Nielsen, P., L. De Kock, C.W. Gowers et al., LIDAR Thomson Scattering for the ITER Core Plasma. Diagnostics for Experimental Thermonuclear Fusion Reactors ed. G.G. 
Peter Stott, Paolo Prandoni and Elio Sindoni. 1998, New York and London: Plenum Press p. 217.

[121] Brown, B.W., C.W. Gowers, P. Nielsen et al., Window transmission monitoring and cleaning schemes used with the Light Detection and Ranging Thomson scattering diagnostic on the JET tokamak. Review of Scientific Instruments, 1995. 66(4): p. 3077-3078.

[122] Smith, O.R.P., C. Gowers, P. Nielsen et al., A self-calibration technique for a Thomson scattering system. Review of Scientific Instruments, 1997. 68: p. 725-727.

[123] Martin, P., A. Morono, and E.R. Hodgson, Surface degradation effects on laser damage in KU1 quartz glass windows for LIDAR applications. Journal of Nuclear Materials, 2002. 307(Part B): p. 1260-1263.

[124] Tartari, U., Collective Thomson Scattering Diagnostic of confined Alpha Particle Distributions in ITER. Diagnostics for Experimental Thermonuclear Fusion Reactors, ed. P.E. Stott, G. Gorini, and E. Sindoni. 1996, New York and London: Plenum Press. p. 513.

[125] Orsitto, F., Review of Possibilities for a Collective Thomson Scattering System on ITER. Diagnostics for Experimental Thermonuclear Fusion Reactors, ed. P.E. Stott, G. Gorini, and E. Sindoni. 1996, New York and London: Plenum Press. p. 527.

[126] Meo, F., H. Bindslev, S.B. Korsholm et al., Design of the collective Thomson scattering diagnostic for International Thermonuclear Experimental Reactor at the 60 GHz frequency range. Review of Scientific Instruments, 2004. 75(10): p. 3585-3588.

[127] Bindslev, H., F. Meo, E.L. Tsakadze et al., Feasibility study of fast ion diagnosis in ITER by collective Thomson scattering, millimeter waves to CO[sub 2] laser. Review of Scientific Instruments, 2004. 75(10): p. 3598-3600.

[128] Donne, A.J.H. and B.C. Schokker, Advantages and Limitations of Microwave Diagnostics in ITER. Diagnostics for Experimental Thermonuclear Fusion Reactors, ed. P.E. Stott, G. Gorini, and E. Sindoni. 1996, New York and London: Plenum Press. p. 215.

[129] Bornatici, M. and U. Ruffina, ECE Diagnostics on ITER. Diagnostics for Experimental Thermonuclear Fusion Reactors, ed. P.E. Stott, G. Gorini, and E. Sindoni. 1996, New York and London: Plenum Press. p. 193.

[130] Hartfuss, H.J., Instrumentation of ECE for ITER. Diagnostics for Experimental Thermonuclear Fusion Reactors, ed. P.E. Stott, G. Gorini, and E. Sindoni. 1996, New York and London: Plenum Press. p. 197.

[131] Hartfuss, H.J., T. Geist, and M. Hirsch, Heterodyne methods in millimetre wave plasma diagnostics with applications to ECE, interferometry and reflectometry. Plasma Physics and Controlled Fusion, 1997. 39(11): p. 1693-1769.

[132] Vayakis, G., T. Ando, N. Bretz et al., The ITER ECE Diagnostic Front End Design, in International School of Plasma Physics "Piero Caldirola" Workshop on Diagnostics 
for Experimental Fusion Reactors 2, ed. P.E. Stott, G. Gorini, P. Prandoni, and E. Sandoni, 1998, New York and London: Plenum Press, p. 185.

[133] Hartfuss, H.J., M. Zerbini, P. Buratti et al. The Non-Imaging Flux Collector as a Compact ECE Antenna for ITER. in International School of Plasma Physics "Piero Caldirola" Workshop on Diagnostics for Experimental Fusion Reactors 2. 1998. New York and London, Plenum Press, New York Conference Proceedings Diagnostics for experimental thermonuclear fusion reactors 2, 189

[134] Bornatici, M. and U. Ruffina, ECE Diagnostic on ITER in the Presence of Suprathermals. 2 ed. Diagnostics for Experimental Thermonuclear Fusion Reactors ed. G.G. Peter Stott, Paolo Prandoni and Elio Sindoni. 1998, New York and London: Plenum Press p. 181.

[135] Vayakis, G., D. Bartlett, P. Edmonds et al., Overview of the ITER Reflectometry Diagnostic System, in International School of Plasma Physics "Piero Caldirola" Workshop on Diagnostics for Experimental Fusion Reactors 2, ed. P.E. Stott, G. Gorini, P. Prandoni, and E. Sandoni, 1998, New York and London: Plenum Press, p. 97.

[136] Laviron, C., A.J.H. Donne, M.E. Manso et al., Reflectometry techniques for density profile measurements on fusion plasmas. Plasma Physics and Controlled Fusion, 1996. 38(7): p. 905-936.

[137] Sabot, R., F. Clairet, J.C. Giacalone et al. Reflectometry: A Reliable And Sensitive Plasma Diagnostic For Density Profile And Turbulence Measurements On ToreSupra. in PLASMA 2005: Int. Conf. on Research and Applications of Plasmas; 3rd German-Polish Conf.on Plasma Diagnostics for Fusion and Applications; 5th FrenchPolish Seminar on Thermal Plasma in Space and Laboratory. 2006. Opole-Turawa (Poland), AIP Conference Proceedings 812, 112-119

[138] Mazzucato, E., Microwave reflectometry for magnetically confined plasmas. Review of Scientific Instruments, 1998. 69(6): p. 2201-2217.

[139] Doyle, E.J., K.W. Kim, J.H. Lee et al., Reflectometry Applications to ITER. Diagnostics for Experimental Thermonuclear Fusion Reactors, ed. P.E. Stott, G. Gorini, and E. Sindoni. 1996, New York and London: Plenum Press. p. 117.

[140] Vershkov, V.A., M.E. Manso, G. Vayakis et al., ITER Reflectometer Diagnostics for the Main Plasma, in International School of Plasma Physics "Piero Caldirola" Workshop on Diagnostics for Experimental Fusion Reactors 2, ed. P.E. Stott, G. Gorini, P. Prandoni, and E. Sandoni, 1998, New York and London: Plenum Press, New York, p. 107.

[141] Veron, D., ITER Reflectometer Diagnostics for the Main Plasma. Infrared and Millinmeter Waves, ed. K.J. Button. Vol. 2. 1979, New York: Academic Press. p. 67.

[142] Snider, R.T., T.N. Carlstrom, C.H. Ma et al., Application of Interferometry and Faraday Rotation Techniques for Density Measurements on ITER. Diagnostics for Experimental Thermonuclear Fusion Reactors, ed. P.E. Stott, G. Gorini, and E. Sindoni. 1996, New York and London: Plenum Press. p. 225. 
[143] Cheon, M.S., Y.U. Nam, J.H. Ha et al., Design of a far-infrared interferometer/polarimeter system for Korea Superconducting Tokamak Advanced Research. Review of Scientific Instruments, 2004. 75(10): p. 3402-3404.

[144] Donne, A.J.H., High spatial resolution interferometry and polarimetry in hot plasmas. Review of Scientific Instruments, 1995. 66(6): p. 3407-3423.

[145] Akiyama, T., K. Tanaka, L.N. Vyacheslavov et al., $\mathrm{CO}_{2}$ laser imaging interferometer for high spatial resolution electron density profile measurements on LHD. Review of Scientific Instruments, 2003. 74(3): p. 1638-1641.

[146] Kawahata, K., K. Tanaka, Y. Ito et al., A two color millimeter-wave interferometer for the measurement of line integral electron density on large helical device. Review of Scientific Instruments, 1999. 70(1): p. 695-698.

[147] Irby, J., R. Murray, P. Acedo et al., A two-color interferometer using a frequency doubled diode pumped laser for electron density measurements. Review of Scientific Instruments, 1999. 70(1): p. 699-702.

[148] Hopf, F.A. and M. Cervantes, Non-Linear Optical Interferometer. Applied Optics, 1982. 21(4): p. 668-677.

[149] Kawano, Y., JT-60, Japan, Private Communication.

[150] Segre, S.E., Plasma Polarimetry for Large Cotton-Mouton and Faraday Effects. Physics of Plasmas, 1995. 2(8): p. 2908-2914.

[151] Giudicotti, L., S.L. Prunty, C. Nyhan et al., A polarization modulation technique for far-infrared polarimetry in large plasmas. Plasma Physics and Controlled Fusion, 2004. 46(4): p. 681-693.

[152] Segre, S.E., Analytic expressions for polarimetry in plasma with large Cotton-Mouton or Faraday effects. Physics of Plasmas, 1996. 3(3): p. 1182-1183.

[153] Fuchs, C. and H.J. Hartfuss, Cotton-Mouton effect measurement in a plasma at the W7-AS stellarator. Physical Review Letters, 1998. 81(8): p. 1626-1629.

[154] Fuchs, C. and H.J. Hartfuss, Line integrated density measurements based on CottonMouton polarimetry. Review of Scientific Instruments, 1999. 70(1): p. 722-725.

[155] Carlstrom, T.N., R.T. Snider, C.B. Baxi et al., Baseline Design of a Multi-Channel Interferometer and Polarimeter for Density Measurements on ITER, in International School of Plasma Physics "Piero Caldirola" Workshop on Diagnostics for Experimental Fusion Reactors 2, ed. P.E. Stott, G. Gorini, P. Prandoni, and E. Sandoni, 1998, New York and London: Plenum Press, p. 193.

[156] Kondoh, T., A.E. Costley, T. Sugie et al., Toroidal interferometer/polarimeter density measurement system on ITER. Review of Scientific Instruments, 2004. 75(10): p. $3420-3422$. 
[157] Donne, A.J.H., M.F. Graswinckel, M. Cavinato et al., Poloidal polarimeter for current density measurements in ITER. Review of Scientific Instruments, 2004. 75(11): p. 4694-4701.

[158] Schweer, B., Atomic beam diagnostics. Fusion Technology, 2000. 37(2T): p. 368-375.

[159] Kornejew, P., W. Bohmeyer, and G. Fussmann, Thermal Helium Beam for Diagnostic of Electron Density and Temperature. Diagnostics for Experimental Thermonuclear Fusion Reactors, ed. P.E. Stott, G. Gorini, and E. Sindoni. 1996, New York and London: Plenum Press. p. 599.

[160] Perfilov, S., A. Melnikov, L. Krupnik et al. Applicability of Heavy Ion Beam Probing for Stellarator W7-X. in PLASMA 2005: Int. Conf. on Research and Applications of Plasmas; 3rd German-Polish Conf.on Plasma Diagnostics for Fusion and Applications; 5th French-Polish Seminar on Thermal Plasma in Space and Laboratory. 2006. Opole-Turawa (Poland), AIP Conference Proceedings 812, 199202

[161] Fujisawa, A., H. Iguchi, A. Taniike et al., A 6 MeV Heavy-Ion Beam Probe for the Large Helical Device. Ieee Transactions on Plasma Science, 1994. 22(4): p. 395-402.

[162] Kislyakov, A.I., V.I. Afanissiev, A.V. Khudoleev et al., NPA at ITER : physical basis and conceptual design, in International School of Plasma Physics "Piero Caldirola" Workshop on Diagnostics for Experimental Fusion Reactors 2, ed. P.E. Stott, G. Gorini, P. Prandoni, and E. Sandoni, 1998, New York and London: Plenum Press, p. 353.

[163] Walker, C.I. and L. De Kock, ITER Plasma Diagnostics generic Access. Diagnostics for Experimental Thermonuclear Fusion Reactors, ed. P.E. Stott, G. Gorini, and E. Sindoni. 1996, New York and London: Plenum Press. p. 39.

[164] Johnson, L.C., C.W. Barnes, R.E. Bell et al., Fusion Product Measurement in D-T Plasmas in TFTR. Diagnostics for Experimental Thermonuclear Fusion Reactors, ed. P.E. Stott, G. Gorini, and E. Sindoni. 1996, New York and London: Plenum Press. p. 369.

[165] Asai, K., T. Iguchi, K. Watanabe et al., Detailed design of ex-vessel neutron yield monitor for ITER. Review of Scientific Instruments, 2004. 75(10): p. 3537-3539.

[166] Elevant, T. and J. Scheffel, Role of Neutron Spectrometers for ITER, in International School of Plasma Physics "Piero Caldirola" Workshop on Diagnostics for Experimental Fusion Reactors 2, ed. P.E. Stott, G. Gorini, P. Prandoni, and E. Sandoni, 1998, New York and London: Plenum Press, p. 429.

[167] Ericsson, G., L. Ballabio, S. Conroy et al., Neutron emission spectroscopy at JET Results from the magnetic proton recoil spectrometer (invited). Review of Scientific Instruments, 2001. 72(1): p. 759-766.

[168] Thomas, P.R., Divertor Diagnostics for JET. Diagnostics for Experimental Thermonuclear Fusion Reactors, ed. P.E. Stott, G. Gorini, and E. Sindoni. 1996, New York and London: Plenum Press. p. 531. 
[169] Leonard, A.W., Diagnostic Needs for Divertor and Edge Physics. Advanced Diagnostics for Magnetic and Inertial Fusion, ed. P.E. Stott, A. Wotton, G. Gorini, E. Sindoni, and D. Batani. 2002, New York: Kluwer Academic/Plenum Publishers. p. 39.

[170] Dore, P. and E. Gauthier. Speckle Interferometry: a Diagnostic for ErosionRedeposition Measurements in Fusion Devices. in 17th International Conference on Plasma Surface Interaction in Controlled Fusion Devices. May 22-26. Hefei, China, Conference Proceedings,

[171] Hodgson, E.R., Radiation Problems and Testing of ITER Diagnostic Components, in International School of Plasma Physics "Piero Caldirola" Workshop on Diagnostics for Experimental Fusion Reactors 2, ed. P.E. Stott, G. Gorini, P. Prandoni, and E. Sandoni, 1998, New York and London: Plenum Press, p. 261.

[172] Yamamoto, S., T. Shikama, V. Belyakov et al., Impact of irradiation effects on design solutions for ITER diagnostics. Journal of Nuclear Materials, 2000. 283-287(Part 1): p. 60-69.

[173] Brichard, B., A.F. Fernandez, H. Ooms et al., Radiation-hardening techniques of dedicated optical fibres used in plasma diagnostic systems in ITER. Journal of Nuclear Materials, 2004. 329-33: p. 1456-1460.

[174] Tanaka, T., A. Suzuki, T. Muroga et al., Radiation induced conductivity of ceramic coating materials under $14 \mathrm{MeV}$ neutron irradiation. Journal of Nuclear Materials, 2004. 329-33: p. 1434-1437.

[175] Shikama, T., T. Nishitani, T. Kakuta et al., Irradiation test of diagnostic components for ITER application in the Japan Materials Testing Reactor. Nuclear Fusion, 2003. 43(7): p. 517-521.

[176] Van Nieuwenhove, R. and L. Vermeeren, Irradiation effects on temperature sensors for ITER application. Review of Scientific Instruments, 2004. 75(1): p. 75-83.

[177] König, R., O. Ogorodnikova, H. Schmidt et al. Development of optical and IR periscopes for quasi-continuous operation of the W7-X stellarator. in 30th EPS Conference on Contr. Fusion and Plasma Phys. 2003. St. Petersburg, Conference Proceedings ECA Vol. 27A, P-4.83

[178] Merrigan, M. and G.A. Wurden, Tokamak Physics Experiment: Diagnostic Window Study. 1995.

[179] Beck, C.M. and C.J. Brearley, CVD diamond as infra-red and optical windows. Gec Review, 1999. 14(2): p. 115-+.

[180] Takahashi, K., K. Sakamoto, T. Imai et al. Development of EC Launcher and Diamond Window for ITER. in IAEA TCM on ECRH Physics and Technology for ITER. 2003. Kloster Seeon, Conference Proceedings,

[181] Thumm, M., R. Heidinger, A. Arnold et al., ITER ECRF Window Development - CVDDiamond Window -. 2002: ITER Task No.: G 55 TT 19 EU (TWO-ECRF/WIN). 
[182] Thumm, M., MPACVD-diamond windows for high-power and long-pulse millimeter wave transmission. Diamond and Related Materials, 2001. 10(9-10): p. 1692-1699.

[183] Thumm, M., MW gyrotron development for fusion plasma applications. Plasma Physics \& Controlled Fusion, 2003. 45(12A): p. 61.

[184] Portafaix, C., V. Basiuk, A. Beraud et al., Design of the infrared monitoring system for CIEL project. Fusion Engineering and Design, 2000. 51-52: p. 1065-1069.

[185] Doane, J.L., Waveguide diamond vacuum windows with Helicoflex seals. 2002: GAA23971.

[186] Lipa, M., C. Portafaix, E. Pluyette et al., Development and testing of diagnostic windows for Tore Supra/CIEL and ITER. Fusion Engineering and Design, 2002. 61-2: p. 801-806.

[187] Missirlian, M., M. Lipa, C. Portafaix et al., Methodology for the design of diagnostic windows for Tore Supra. Fusion Engineering and Design, 2003. 66-8: p. 911-917.

[188] Ogorodnikova, O.V., R. König, A. Pospieszczyk et al., Thermo-stress analysis of actively cooled diagnostic windows for quasi-continuous operation of the W7-X stellarator. Journal of Nuclear Materials, 2005. 341(2-3): p. 175-183.

[189] Guilhem, D., J.L. Bondil, B. Bertrand et al., Tore-Supra infrared thermography system, a real steady-state diagnostic. Fusion Engineering and Design, 2005. 74(1-4): p. $879-883$.

[190] Yoshimura, Y., S. Kubo, T. Shimozuma et al., Achievement of One Hour Discharge with ECH on LHD. Journal of Physics: Conference Series, 2005: p. 189-197.

[191] König, R., K. Grosser, D. Hildebrandt et al., Development of an actively cooled periscope head suitable for divertor observation during quasi-continuous operation of the W7-X stellarator. Fusion Engineering and Design, 2005. 74(1-4): p. 751-755.

[192] Bates, S.C., Low Loss Sapphire Windows for High Power Microwave Transmission. 1999: p. US DOE Contract \# DE-FG02-95ER86038.

[193] Harris, D.C., F. Schmid, D.R. Black et al., Factors that influence mechanical failure of sapphire at high temperatures. Proceedings of SPIE - the International Society for Optical Engineering, 1997: p. 226-35.

[194] Cook, R.L., M. Kochis, I.E. Reimanis et al. A New Powder Production Route for Transparent Spinel Windows: Powder Synthesis and Window Properties. in International Society for Optical Engineering (SPIE) Defense and Security Symposium 2005: Window and Dome Technologies and Materials IX. 2005. Orlando, FL, Conference Proceedings,

[195] Touloukian, Y.S., Thermophysical Properties of high temperature solid materials Y S Touloukian, ed. 1967, New York: Macmillan. 
[196] Klocek, P., Handbook of infrared optical materials. Optical engineering 30. 1991, New York.

[197] Klein, C.A., B. Dibenedetto, and J. Pappis, Zns, Znse, and Zns/Znse Windows - Their Impact on Flir System Performance. Optical Engineering, 1986. 25(4): p. 519-531.

[198] Pickles, C.S.J. and J.E. Field, The Laboratory Simulation of Thermal Shock Failure. Journal of Physics D-Applied Physics, 1996. 29(2): p. 436-441.

[199] Lanin, A.G., E.L. Muravin, V.P. Popov et al., Thermal shock resistance and thermalmechanical processing of sapphire. Journal of the European Ceramic Society, 2003. 23(3): p. 455-68.

[200] Zaccaria, P. and S. Bello, Thermal and mechanical behaviour of diagnostic windows subjected to intense heat fluxes. Fusion Engineering and Design, 2000. 49-50: p. 417.

[201] Hearn, D.R. Vacuum window optical power induced by temperature gradients. in Proceedings of SPIE, Earth Observing Systems IV. 1999, Conference Proceedings 3750, 297-308

[202] Harris, D.C., Infrared Window and dome materials. Tutorial texts in Optical Engineering, ed. SPIE. Vol. TT 10. 1992, Bellingham, Washington: SPIE Optical Engineering Press. p. 175.

[203] Zelikin, Y.M., N.K. Morozova, V.G. Galstyan et al., Optical properties of various structural forms of zinc oxide. Zhurnal Prikladnoi Spektroskopii, 1990. 53(3): p. 46470.

[204] Arenas, O.L., M.T.S. Nair, and P.K. Nair, Chemical bath deposition of ZnS thin films and modification by air annealing. Semiconductor Science and Technology, 1997(10): p. $1323-1330$.

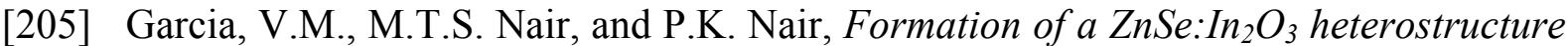
by air annealing ZnSe-In thin film. Semiconductor Science \& Technology, 1999. 14(4): p. 366-72.

[206] Omega, Non-Contact Temperature Measurement. Transactions in Measurement and Control. Vol. 1. 1998: Putman Publishing Company and Omega Press LLC. 83.

[207] Tiernan, R.J. and J.E. Saunders, Normal Emittance Measurements by a Transient Temperature Technique. Journal of Applied Physics, 1988. 64(2): p. 459-463.

[208] Thomas, M.E., R.I. Joseph, and W.J. Tropf, Infrared Transmission Properties of Sapphire, Spinel, Yttria, and Alon as a Function of Temperature and Frequency. Applied Optics, 1988. 27(2): p. 239-245.

[209] Gillespie, D.T., A.L. Olsen, and L.W. Nichols, Transmittance of Optical Materials at High Temperatures in 1-Mu to 12-Mu Range. Applied Optics, 1965. 4(11): p. 1488-\&.

[210] Freeland, C.M., High temperature transmission measurements of IR window materials. SPIE, 1988. 929: p. 79. 
[211] Cox, J.A., D. Greenlaw, G. Terry et al., Comparative study of advanced IR transmissive materials. SPIE 1986. 683: p. 49.

[212] Ghosh, G., Handbook of Thermo-Optic Coefficients of Optical Materials with Applications, ed. 1998, Academic Press, San Diego, p. 325.

[213] Sato, F., T. Iida, Y. Oyama et al., Photon emission induced by fusion neutrons on optical window materials. Journal of Nuclear Materials, 1998. 258-263(Part 2): p. 1897-1901.

[214] Ishitsuka, E., H. Sagawa, A. Nagashima et al., Neutron Irradiation Test of Optical Components for Fusion Reactor, in Effects of Radiation on Materials: 19th International Symposium ASTM STP Ser., ed. M.L. Hamilton, A. Kumar, S.T. Rosinski, and M.L. Grossbeck, 2000, West Conshohocken: American Society for Testing \& Materials, p. p. 1176.

[215] Morono, A. and E.R. Hodgson, Oxygen interstitial trapping in electron irradiated sapphire. Journal of Nuclear Materials, 2002. 307-311(Part 2): p. 1246-1249.

[216] Flerov, A. and V. Flerov, Afterglow of heavily irradiated sapphire. Nuclear Instruments and Methods in Physics Research Section B: Beam Interactions with Materials and Atoms, 1998. 141(1-4): p. 378-380.

[217] Morono, A. and E.R. Hodgson, On the origin of the F+centre radioluminescence in sapphire. Journal of Nuclear Materials, 1997. 249(2-3): p. 128-132.

[218] Morono, A. and E.R. Hodgson, Enhanced oxygen vacancy aggregation and colloid production in Al2O3. Journal of Nuclear Materials, 1997. 250(2-3): p. 156-163.

[219] Morono, A. and E.R. Hodgson, Radioluminescence problems for diagnostic windows. Journal of Nuclear Materials, 1995. 224(3): p. 216-221.

[220] Orlinski, D.V., Investigations of materials for windows of the fusion reactor diagnostic systems. Plasma Devices \& Operations, 2001. 9(3-4): p. 173-209.

[221] Morono, A., R. Vila, and E.R. Hodgson, KU1 and KS-4V quartz glass lenses for remote handling and diagnostic optical transmission systems. Journal of Nuclear Materials, 2004. 329-33: p. 1438-1441.

[222] Garcia-Matos, M., A. Morono, and E.R. Hodgson, KU1 quartz glass for remote handling and LIDAR diagnostic optical transmission systems. Journal of Nuclear Materials, 2000. 283-287(pt.B): p. 890-3.

[223] Orlinski, D.V., I.V. Al'tovsky, T.A. Bazilevskaya et al., Preliminary results of window radiation resistance investigations. Journal of Nuclear Materials, 1994. 212-215(Part 2): p. 1059-1064.

[224] Martín, P., M. León, and A. Ibarra, Photoluminescence in neutron irradiated fused silica. physica status solidi (c), 2005. 2(1): p. 624-628. 
[225] Yoshida, T., T. Ii, T. Tanabe et al., In situ luminescence and optical absorption measurements of silica in reactor core. Journal of Nuclear Materials, 2002. 307311(Part 2): p. 1268-1272.

[226] Cooke, D.W. and B.L. Bennett, Optical absorption and luminescence of 14-MeV neutron-irradiated CaF2 single crystals. Journal of Nuclear Materials, 2003. 321(2-3): p. $158-164$.

[227] Martin, P., A. Morono, and E.R. Hodgson, Radiation effects on laser damage in KU1 quartz glass. Journal of Nuclear Materials, 2000. 283-287(Part 2): p. 894-897.

[228] Voitsenya, V.S., Analysis of the mechanism and source of contamination of diagnostic windows in fusion devices. Journal of Nuclear Materials, 1998. 258-263(pt.A): p. 65861.

[229] Yamamura, Y., Y. Itikawa, and N. Itoh, Angular Dependence of sputtering yields of monoatomic solids. 1983.

[230] König, R.W.T., H. Anderson, P. Breger et al. Active spectroscopy on JET and ITER. in International School of Plasma Physics "Piero Caldirola" Workshop on Diagnostics for Experimental Fusion Reactors 2. 1998. New York and London, Plenum Press Conference Proceedings Diagnostics for experimental thermonuclear fusion reactors 2, 371

[231] Morsi, H.W., M. vonHellermann, R.W.T. Konig et al., A visible and UV charge exchange spectroscopy system for the tritium phase of JET. Plasma Physics and Controlled Fusion, 1995. 37(12): p. 1407-1431.

[232] Martin, P., A. Morono, and E.R. Hodgson, Surface degradation effects on laser damage in KU1 quartz glass windows for LIDAR applications. Journal of Nuclear Materials, 2002. 307-311(Part 2): p. 1260-1263.

[233] Martin, P., A. Morono, and E.R. Hodgson, Laser induced damage enhancement due to stainless steel deposition on KS-4V and KU1 quartz glasses. Journal of Nuclear Materials, 2004. 329-333(Part 2): p. 1442-1445.

[234] Gowers, C.W., M. Beurskens, and P. Nielsen, Higher Spatial Resolution LIDAR Thomson Scattering at JET. Journal of Plasma and Fusion Research, 2000. 76: p. 974877.

[235] Johnson, D. and B. Grek, ITER Edge Thomson Scattering. Diagnostics for Experimental Thermonuclear Fusion Reactors ed. G.G. Peter Stott, Paolo Prandoni and Elio Sindoni. Vol. 2. 1998, New York and London: Plenum Press p. 227.

[236] Razdobarin, G.T. and E.E. Mukhin, Status of Thomson Scattering Diagnostic Design for ITER X-Point and Divertor Plasmas. Diagnostics for Experimental Thermonuclear Fusion Reactors ed. G.G. Peter Stott, Paolo Prandoni and Elio Sindoni. Vol. 2. 1998, New York and London: Plenum Press p. 237. 
[237] Kochergin, M.M., E.E. Mukhin, G.T. Razdobarin et al., Interfaces for Thomson scattering diagnostics in the Divertor and SOL near the X-point plasmas of ITER-feat. Plasma Devices and Operations, 2003. 11(1): p. 1-6.

[238] McNeill, D.H., Effect of changes in viewing window transmission on high-temperature Thomson scattering data. Review of Scientific Instruments, 1990. 61(4): p. 12631266.

[239] Jacob, W., Redeposition of hydrocarbon layers in fusion devices. Journal of Nuclear Materials, 2005. 337-39(1-3): p. 839-846.

[240] Jacob, W., Surface reactions during growth and erosion of hydrocarbon films. Thin Solid Films, 1998. 326(1-2): p. 1-42.

[241] Maruyama, K., W. Jacob, and J. Roth, Erosion behavior of soft, amorphous deuterated carbon films by heat treatment in air and under vacuum. Journal of Nuclear Materials, 1999. 264(1-2): p. 56-70.

[242] Schwarz-Selinger, T., A. von Keudell, and W. Jacob, Plasma chemical vapor deposition of hydrocarbon films: The influence of hydrocarbon source gas on the film properties. Journal of Applied Physics, 1999. 86(7): p. 3988-96.

[243] Keudell, A.v., C. Hopf, T. Schwarz-Selinger et al., Surface loss probabilities of hydrocarbon radicals on amorphous hydrogenated carbon film surfaces: Consequences for the formation of re-deposited layers in fusion experiments. Nuclear Fusion, 1999(10): p. 1451-1462.

[244] Coad, J.P., N. Bekris, J.D. Elder et al., Erosion/deposition issues at JET. Journal of Nuclear Materials, 2001. 290-293: p. 224-230.

[245] Esser, H.G., V. Philipps, M. Freisinger et al., Effect of plasma configuration on carbon migration measured in the inner divertor of JET using quartz microbalance. Journal of Nuclear Materials, 2005. 337-339: p. 84-88.

[246] Yoshida, H., O. Naito, T. Hatae et al., Approach to a window coating problem by in situ transmission monitoring and laser blow-off cleaning developed in the JT-60U Thomson scattering system. Review of Scientific Instruments, 1997. 68(1): p. 256257.

[247] Wang, W., W. Jacob, and J. Roth, Oxidation and hydrogen isotope exchange in amorphous, deuterated carbon films. Journal of Nuclear Materials, 1997. 245(1): p. 66-71.

[248] Ventura, S.D., E.G. Birgin, J.M. Martinez et al., Optimization techniques for the estimation of the thickness and the optical parameters of thin films using reflectance data. Journal of Applied Physics, 2005. 97(4): p. 043512-12.

[249] Birgin, E.G., I. Chambouleyron, and J.M. Martinez, Estimation of the optical constants and the thickness of thin films using unconstrained optimization. Journal of Computational Physics, 1999. 151(2): p. 862-880. 
[250] Birgin, E.G., I.E. Chambouleyron, J.M. Martinez et al., Estimation of optical parameters of very thin films. Applied Numerical Mathematics, 2003 . 47(2): p. 109119.

[251] Skinner, C.H., H. Kugel, A.L. Roquemore et al., Time resolved deposition measurements in NSTX. Journal of Nuclear Materials, 2005: p. 129-33.

[252] Rohde, V., M. Mayer, J. Likonen et al., Carbon erosion and a:C-H layer formation at ASDEX Upgrade. Journal of Nuclear Materials, 2005. 337-339: p. 847-851.

[253] Okazaki, K., H. Oyama, Y. Ishibe et al., Influence of Insitu Carbon Coatings on Window Materials in Fusion-Experimental Devices. Journal of Nuclear Materials, 1987. 147: p. 761-764.

[254] Brown, B.C., C.W. Gowers, P. Nielsen et al., WindowTransmission Monitoring and Cleaning Schemes used with the LIDAR Thomson Scattering Diagnostic on the JET Tokamak. 1994.

[255] Gonzalez, S.M., A. Morono, and E.R. Hodgson, Optical and electrical degradation of $H+$ implanted $K S-4 V$ quartz glass. Fusion Engineering and Design, 2005. 74(1-4): p. 831-834.

[256] Counsell, G.F. and C.H. Wu, In-situ detection and removal of carbon debris - a challenge for the next-step fusion device. Physica Scripta, 2001. T91: p. 70-75.

[257] Narihara, K. and S. Hirokura, Cleaning of Thomson scattering window by a laser blow-off method. Review of Scientific Instruments, 1992. 63(6): p. 3527-3528.

[258] Gibson, K.J., G.F. Counsell, C. Curran et al., The removal of co-deposited hydrocarbon films from plasma facing components using high-power pulsed flashlamp irradiation. Journal of Nuclear Materials, 2005. 337-339: p. 565-569.

[259] Gubbini, E., G. Kommol, M. Schnurer et al., On-line cleaning of optical components in a multi-TW-Ti:Sa laser system. Vacuum, 2004. 76(1): p. 45-49.

[260] Park, S.-G., C. Kim, and B.-h. O, An array of inductively coupled plasma sources for large area plasma. Thin Solid Films, 1999. 355-356: p. 252-255.

[261] Orlinski, D.V., Radiation Hardening of Diagnostic Components. Diagnostics for Experimental Thermonuclear Fusion Reactors, ed. P.E. Stott, G. Gorini, and E. Sindoni. 1996, New York and London: Plenum Press. p. 59.

[262] Lipa, M., B. Schunke, and C. Gil, First Mirror Studies in Tore Supra. 2004: EFDA Ref.: TW2-TPDS-DIADEV-D02.

[263] Mayer, M., R. Behrisch, C.W. Gowers et al., Change of Optical Reflectivity of Mirror Surfaces Exposed to JET Plasmas, in International School of Plasma Physics "Piero Caldirola" Workshop on Diagnostics for Experimental Fusion Reactors 2, ed. P.E. Stott, G. Gorini, P. Prandoni, and E. Sandoni, 1998, New York and London: Plenum Press, p. 279. 
[264] Linke, J.M., T. Hirai, M. Rodig et al., Performance of plasma-facing materials under intense thermal loads in tokamaks and stellarators. Fusion Science and Technology, 2004. 46(1): p. 142-151.

[265] Voitsenya, V.S., V.G. Konovalov, A.F. Shtan et al., Some problems of the material choice for the first mirrors of plasma diagnostics in a fusion reactor. Review of Scientific Instruments, 1999. 70(1): p. 790-793.

[266] Verbeek, H., J. Stober, D.P. Coster et al., Interaction of charge exchange neutrals with the main chamber walls of plasma machines. Nuclear Fusion, 1998. 38(12): p. 17891803.

[267] Voitsenya, V.S., A.J.H. Donne, A.F. Bardamid et al., Simulation of environment effects on retroreflectors in ITER. Review of Scientific Instruments, 2005. 76(8): p. 083502 .

[268] Malaquias, A., M. von Hellermann, S. Tugarinov et al., Active beam spectroscopy diagnostics for ITER: Present status (invited). Review of Scientific Instruments, 2004. 75(10): p. 3393-3398.

[269] von Hellermann, M., M. de Bock, R. Jaspers et al., Pilot experiments for the International Thermonuclear Experimental Reactor active beam spectroscopy diagnostic. Review of Scientific Instruments, 2004. 75(10): p. 3458-3461.

[270] Kuldkepp, M., E. Rachlew, N.C. Hawkes et al., First mirror contamination studies for polarimetry motional Stark effect measurements for ITER. Review of Scientific Instruments, 2004. 75(10): p. 3446-3448.

[271] Voitsenya, V.S., A. Sagara, A.I. Belyaeva et al., Effect of exposure inside the Large Helical Device vessel on the optical properties of stainless steel mirrors. Plasma Devices and Operations, 2005. 13(4): p. 291-300.

[272] Ramsey, A.T. and K.W. Hill, Nuclear radiation effects in fused $\mathrm{SiO}_{2}$ lightguides. Review of Scientific Instruments, 1992. 63(10): p. 4735-4737.

[273] Morgan, P.D. Irradiation of optical fibres at JET through $14 \mathrm{MeV}$ Neutron Production. in Fusion Technology. 1992. Rome, Italy, North-Holland, 1993 Conference Proceedings, 722

[274] Friebele, E.J., C.G. Askins, M.E. Gingerich et al., Optical fiber waveguides in radiation environments, II. Nuclear Instruments and Methods in Physics Research Section B: Beam Interactions with Materials and Atoms, 1984. 1(2-3): p. 355-369.

[275] Ramsey, A.T., W. Tighe, J. Bartolick et al., Radiation effects on heated optical fibers. Review of Scientific Instruments, 1997. 68(1): p. 632-635.

[276] Maas, A.C., P. Andrew, P. Coad et al., Diagnostic experience during deuteriumtritium experiments in JET, techniques and measurements. Fusion Engineering and Design, 1999. 47(2-3): p. 247-265. 
[277] Sporea, D.G. and R.A. Sporea, Setup for the in situ monitoring of the irradiationinduced effects in optical fibers in the ultraviolet-visible optical range. Review of Scientific Instruments, 2005. 76(11): p. 113110-5.

[278] Brichard, B., P. Borgermans, A.F. Fernandez et al., Radiation effect in silica optical fiber exposed to intense mixed neutron-gamma radiation field. IEEE Transactions on Nuclear Science, 2001. 48(6 Part 1): p. 2069-2073.

[279] Marrone, M.J., Radiation-induced luminescence in silica core optical fibers. Applied Physics Letters, 1981. 38(3): p. 115-117.

[280] Siegel, G.H., IEEE Trans. Nucl. Sci. NS, 1981. 28: p. 4095.

[281] Herschel, H. and O. Kohn. Regeneration of irradiated optical fibres by photobleaching. in Fifth European Conference on Radiation and its Effects on Components and Systems. 1999. Fontevraud, France, Conference Proceedings, 502507

[282] Vayakis, G., C. Walker, and I.I.T.P. Teams, Magnetic diagnostics for ITER/BPX plasmas (invited). Review of Scientific Instruments, 2003. 74(4): p. 2409-2417.

[283] Hodgson, E.R. and S. Clement, Dose-Rate Dependence of the Radiation-Induced Electrical-Conductivity in Mgo. Journal of Nuclear Materials, 1988. 155: p. 357-360.

[284] Kinoshita, C. and S.J. Zinkle, Potential and limitations of ceramics in terms of structural and electrical integrity in fusion environments. Journal of Nuclear Materials, 1996: p. 100-10.

[285] Bender, S.E., V.M. Chernov, P.V. Demenkov et al., Investigation of RIEMF nature in magnetic sensors for ITER. Fusion Engineering and Design, 2001: p. 911-15.

[286] Vermeeren, L. and R. Van Nieuwenhove, Theoretical study of radiation induced electromotive force effects on mineral insulated cables. Review of Scientific Instruments, 2003. 74(11): p. 4667-4674.

[287] Van Nieuwenhove, R. and L. Vermeeren, Experimental study of radiation induced electromotive force effects on mineral insulated cables. Review of Scientific Instruments, 2003. 74(11): p. 4675-4682.

[288] Vila, R. and E.R. Hodgson, RIEMF in $\mathrm{MgO}$ and Al2O3 insulated MI cable ITER magnetic diagnostic coils. Journal of Nuclear Materials, 2004. 329-33: p. 1524-1528.

[289] Bender, S.E., P.V. Demenkov, O.A. Plaksin et al., Effect of the dose rate and temperature on radiation-induced charge separation in cables with mineral insulation under pulsed reactor irradiation. Instruments and Experimental Techniques, 2004. 47: p. 163.

[290] Nishitani, T., G. Vayakis, M. Yamauchi et al., Radiation-induced thermoelectric sensitivity in the mineral-insulated cable of magnetic diagnostic coils for ITER. Journal of Nuclear Materials, 2004. 329-33: p. 1461-1465. 
[291] Vayakis, G., T. Sugie, T. Kondoh et al., Radiation-induced thermoelectric sensitivity (RITES) in ITER prototype magnetic sensors. Review of Scientific Instruments, 2004. 75(10): p. 4324-4327.

[292] Lazzaro, E., Magnetic Equilbrium Reconstruction Techniques for Tokamak Reactors. Diagnostics for Experimental Thermonuclear Fusion Reactors, ed. P.E. Stott, G. Gorini, and E. Sindoni. 1996, New York and London: Plenum Press. 83.

[293] Takeda, T. and S. Tokuda, Computation of Mhd Equilibrium of Tokamak Plasma. Journal of Computational Physics, 1991. 93(1): p. 1-107.

[294] Braams, B.J., The Interpretation of Tokamak Magnetic Diagnostics. Plasma Physics and Controlled Fusion, 1991. 33(7): p. 715-748.

[295] Zwingmann, W., Equilibrium analysis of steady state tokamak discharges. Nuclear Fusion, 2003. 43(9): p. 842-850.

[296] Moreau, P., P. Defrasne, E. Joffrin et al., A magnetic diagnostic on Tore Supra. Review of Scientific Instruments, 2003. 74(10): p. 4324-4330.

[297] Jaenicke, R., E. Ascasibar, P. Grigull et al., Detailed Investigation of the Vacuum Magnetic-Surfaces on the W7-AS Stellarator. Nuclear Fusion, 1993. 33(5): p. 687-704.

[298] Zarnstorff, M.C., L.A. Berry, A. Brooks et al., Physics of the compact advanced stellarator NCSX. Plasma Physics and Controlled Fusion, 2001. 43: p. A237-A249.

[299] Lazarus, E.A., M.C. Zarnstorff, S.R. Hudson et al., Simulation of a discharge for the NCSX stellarator. Fusion Science and Technology, 2004. 46(1): p. 209-214.

[300] Yamaguchi, T., K.Y. Watanabe, S. Sakakibara et al., Measurement of anisotropic pressure using magnetic measurements in LHD. Nuclear Fusion, 2005. 45(11): p. L33-L36.

[301] Zwingmann, W., L.G. Eriksson, and P. Stubberfield, Equilibrium analysis of tokamak discharges with anisotropic pressure. Plasma Physics and Controlled Fusion, 2001. 43(11): p. 1441-1456.

[302] De Kock, L., S. Ali-Arshad, V. Belyakov et al., Overview of Mangnetic Diagnostic Planned for ITER. Diagnostics for Experimental Thermonuclear Fusion Reactors, ed. P.E. Stott, G. Gorini, and E. Sindoni. 1996, New York and London: Plenum Press. 71.

[303] Spuig, P., P. Defrasne, G. Martin et al., An analog integrator for thousand second long pulses in Tore Supra. Fusion Engineering and Design, 2003. 66-8: p. 953-957.

[304] Deranian, R.D., J.R. Ferron, D.A. Humphreys et al., Integrated plasma control in nextgeneration devices using DIII-D modeling and simulation approaches. Fusion Science and Technology, 2005. 47(3): p. 768-773.

[305] Jhang, H. and J.Y. Kim, Magnetics control simulations for KSTAR plasmas. Fusion Engineering and Design, 2002. 60(3): p. 397-401. 
[306] Jhang, H.G., C.E. Kessel, N. Pomphrey et al., Simulation studies of plasma shape identification and control in Korea Superconducting Tokamak Advanced Research. Fusion Engineering and Design, 2001. 54(1): p. 117-134.

[307] Moret, J.M., F. Buhlmann, D. Fasel et al., Magnetic measurements on the TCV Tokamak. Review of Scientific Instruments, 1998. 69(6): p. 2333-2348.

[308] Shchepetov, S.V., J.A. Jimenez, and A.B. Kuznetsov, Stellarator magnetic analysis: resolvability problems. Plasma Physics and Controlled Fusion, 2001. 43(4): p. 417427.

[309] Pustovitov, V.D., Magnetic diagnostics: General principles and the problem of reconstruction of plasma current and pressure profiles in toroidal systems. Nuclear Fusion, 2001. 41(6): p. 721-730.

[310] Wesley, J., H.W. Bartels, D. Boucher et al., Operation and control of ITER plasmas. Nuclear Fusion, 2000. 40(3Y): p. 485-494.

[311] Kolesnichenko, Y.I., V.V. Lutsenko, H. Wobig et al., Alfven instabilities driven by circulating ions in optimized stellarators and their possible consequences in a Helias reactor. Physics of Plasmas, 2002. 9(2): p. 517-528.

[312] McCormack, B., R. Kaita, H. Kugel et al., Rogowski loop designs for NSTX. IEEE, 1999: p. 306-9.

[313] Edlington, T., R. Martin, and T. Pinfold, MAST magnetic diagnostics. Review of Scientific Instruments, 2001. 72(1): p. 421-425.

[314] Lee, S.G. and J.G. Bak, Magnetic diagnostics for Korea superconducting tokamak advanced research. Review of Scientific Instruments, 2001. 72(1): p. 439-441.

[315] Moret, J.M., F. Buhlmann, and G. Tonetti, Fast single loop diamagnetic measurements on the TCV tokamak. Review of Scientific Instruments, 2003. 74(11): p. 4634-4643.

[316] Greuner, H., J. Glagla, O. Jandl et al., Concepts and prototype elements of the low-Z wall protection for the stellarator W7-X. Fusion Engineering and Design, 2001. 56-7: p. 297-302.

[317] Schittenhelm, M., H. Zohm, and ASDEX_Upgrade_Team, Analysis of coupled MHD modes with Mirnov probes in ASDEX upgrade. Nuclear Fusion, 1997. 37(9): p. 12551270.

[318] Wong, H., Low-frequency noise study in electron devices: review and update. Microelectronics Reliability, 2003. 43(4): p. 585-599.

[319] Hutchinson, I.H., Principles of Plasma Diagnostics. 1987: Cambridge University Press. 
[320] Bak, J.G., S.G. Lee, D. Son et al., Performance of the magnetic sensor and the integrator for the KSTAR magnetic diagnostics. Review of Scientific Instruments, 2004. 75(10): p. 4305-4307.

[321] Sudo, S., Y. Nagayama, B.J. Peterson et al., Recent diagnostic developments on LHD. Plasma Physics and Controlled Fusion, 2003. 45: p. A425-A443.

[322] Sakakibara, S. Magnetic Measurements in LHD. in 3rd Validation Workshop. 2004. Cadarache, France, Conference Proceedings,

[323] Kawarnata, Y., I. Yonekawa, and K. Kurihara, Development of an intelligent digital integrator for long-pulse operation in a tokamak. IEEE, 2002: p. 172-5.

[324] Strait, E.J., J.D. Broesch, R.T. Snider et al., A hybrid digital-analog long pulse integrator. Review of Scientific Instruments, 1997. 68(1): p. 381.

[325] Werner, A., W7-X magnetic diagnostics: Performance of the digital integrator. Review of Scientific Instruments, 2006. 77(1): p. scheduled for publication.

[326] Duran, I., J. Stockel, G. Mank et al., Measurements of magnetic field fluctuations using an array of Hall detectors on the TEXTOR tokamak. Review of Scientific Instruments, 2002. 73(10): p. 3482-3489.

[327] Liu, Y.H., D.A. Maurer, G.A. Navratil et al., High spatial resolution Hall sensor array for edge plasma magnetic field measurements. Review of Scientific Instruments, 2005. 76(9): p. 093501.

[328] Woolley, R.D., Tokamak poloidal magnetic field measurements accurate for unlimited time durations. IEEE, 1995. 2: p. 1530-3.

[329] Woolley, R.D., Progress on a steady-state tokamak magnetic field sensor: accurate for unlimited time durations for fusion reactors. IEEE, 2003: p. 351-8.

[330] Fujita, J., K. Kawahata, K. Matsuura et al., A Hybrid Magnetic Probe for Steady State Magnetic Field Measurements. Diagnostics for Experimental Thermonuclear Fusion Reactors, ed. P.E. Stott, G. Gorini, and E. Sindoni. 1996, New York and London: Plenum Press. 103.

[331] Shi, Y.J., Improved signal analysis for the calibration of the motional Stark effect diagnostic. Review of Scientific Instruments, 2006. 77(2): p. 023503.

[332] Jayakumar, R.J., S.L. Allen, K.H. Burrell et al., Current profile measurement on the DIII-D tokamak. Fusion Science and Technology, 2005. 48(2): p. 852-863.

[333] Segre, S.E., On the Simultaneous Measurement by Polarimetry of Both Electron Line Density and Poloidal Magnetic-Field in Tokamaks. Plasma Physics and Controlled Fusion, 1993. 35(9): p. 1261-1269.

[334] Alasia, D. and L. Thevenaz, A novel all-fibre configuration for a flexible polarimetric current sensor. Measurement Science \& Technology, 2004. 15(8): p. 1525-1530. 
[335] Osakabe, N., T. Matsuda, T. Kawasaki et al., Experimental Confirmation of AharonovBohm Effect Using a Toroidal Magnetic-Field Confined by a Superconductor. Physical Review A, 1986. 34(2): p. 815-822.

[336] Kuznetsov, Y.K. and I.V. Yasin, Fast and Accurate Methods of Plasma Boundary Determination in ITER from External Magnetic Measurements. Diagnostics for Experimental Thermonuclear Fusion Reactors, ed. P.E. Stott, G. Gorini, and E. Sindoni. 1996, New York and London: Plenum Press. p. 97.

[337] Lao, L.L., H.E. St John, Q. Peng et al., MHD equilibrium reconstruction in the DIII-D tokamak. Fusion Science and Technology, 2005. 48(2): p. 968-977.

[338] Ferron, J.R., M.L. Walker, L.L. Lag et al., Real time equilibrium reconstruction for tokamak discharge control. Nuclear Fusion, 1998. 38(7): p. 1055-1066.

[339] Hirshman, S.P. and J.C. Whitson, Steepest-Descent Moment Method for 3Dimensional Magnetohydrodynamic Equilibria. Physics of Fluids, 1983. 26(12): p. 3553-3568.

[340] Suzuki, Y., Y. Nakamura, K. Kondo et al., MHD equilibrium of a low-shear helical axis Heliotron. Plasma Physics and Controlled Fusion, 2003. 45(6): p. 971-981.

[341] Drevlak, M., D. Monticello, and A. Reiman, PIES free boundary stellarator equilibria with improved initial conditions. Nuclear Fusion, 2005. 45(7): p. 731-740.

[342] Dinklage, A., J. Geiger, G. Kuhner et al., A Magnetic Configuartion Database for Wendelstein 7-X and its Application Programming Interface. Fusion Engineering \& Design, 2006: p. accepted for publication.

[343] Gardner, H.J., Modeling the Behavior of the Magnetic-Field Diagnostic Coils on the W7-AS Stellarator Using a 3-Dimensional Equilibrium Code. Nuclear Fusion, 1990. 30(8): p. 1417-1424.

[344] Hirshman, S.P., E.A. Lazarus, J.D. Hanson et al., Magnetic diagnostic responses for compact stellarators. Physics of Plasmas, 2004. 11(2): p. 595-603.

[345] Callaghan, H.P., P.J. McCarthy, and J. Geiger, Fast equilibrium interpretation on the W7-AS stellarator using function parameterization. Nuclear Fusion, 1999. 39(4): p. 509-523.

[346] Callaghan, H.P., P.J. McCarthy, and J. Geiger, Pressure profile recovery on W7-AS with idealized external magnetic measurements using function parametrization. Plasma Physics and Controlled Fusion, 2000. 42(10): p. 1013-1022.

[347] Sengupta, A., P.J. McCarthy, J. Geiger et al., Fast recovery of vacuum magnetic configuration of the W7-X stellarator using function parametrization and artificial neural networks. Nuclear Fusion, 2004. 44(11): p. 1176-1188.

[348] Kim, J.S., M.S. Chance, D.H. Edgell et al., Phase folding of magnetic signals in tokamak plasmas. Plasma Physics and Controlled Fusion, 2001. 43(11): p. 1593-1613. 
[349] Zegenhagen, S., A. Werner, A. Weller et al., Analysis of Alfven Eigenmodes in stellarators using non-evenly spaced probes. Plasma Physics \& Controlled Fusion, 2006: p. accepted for publication. 


\section{Figures}

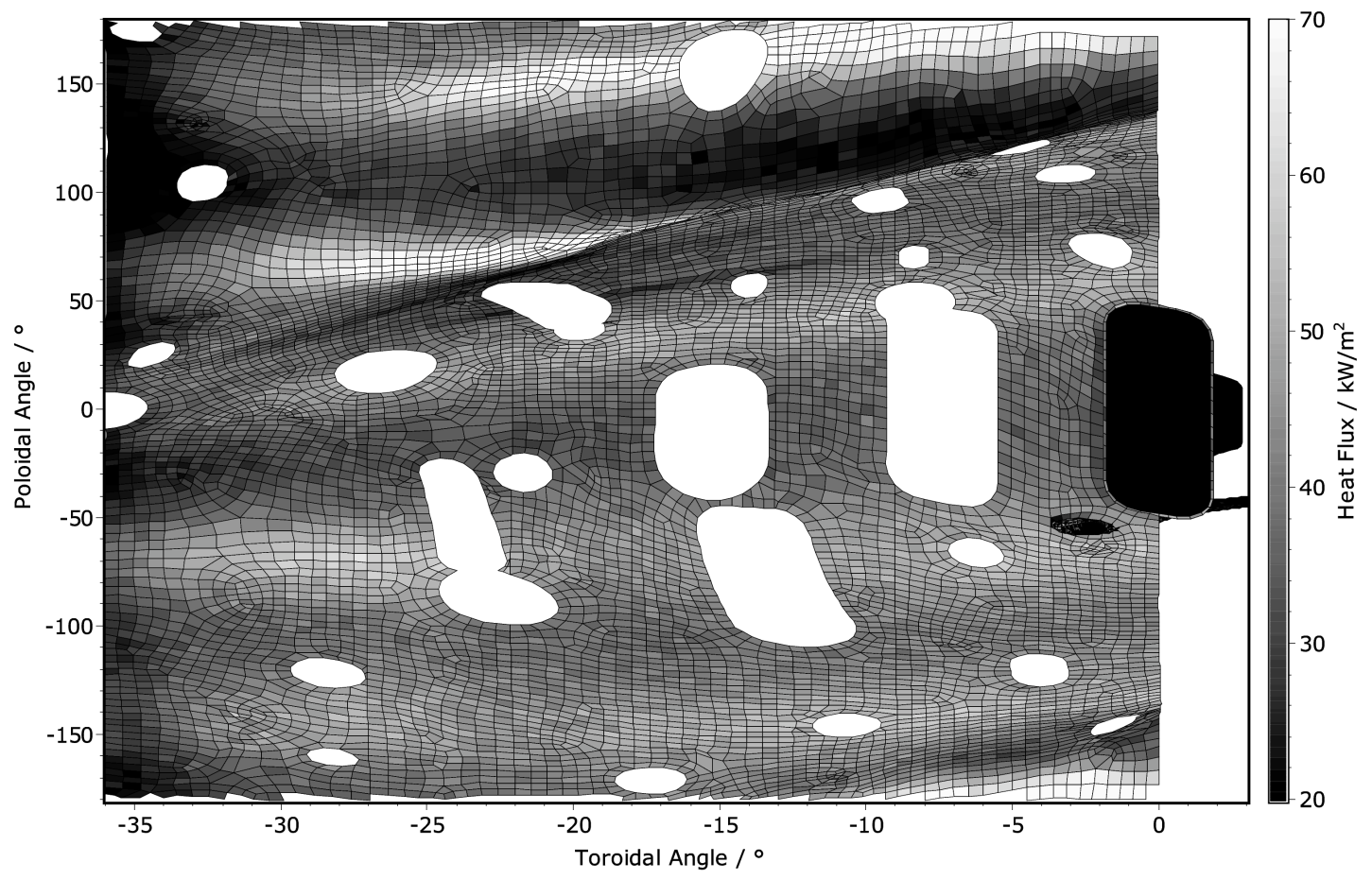

Figure 1: Unrolled view of the plasma radiation load distribution for concentrated $X$-line emission onto the W7-X vessel and ports. Shadowing target and baffle plates of the divertor are not shown. The average load is in the order of $40 \mathrm{~kW} / \mathrm{m}^{2}$ with localized maxima of up to $80 \mathrm{~kW} / \mathrm{m}^{2}$. 


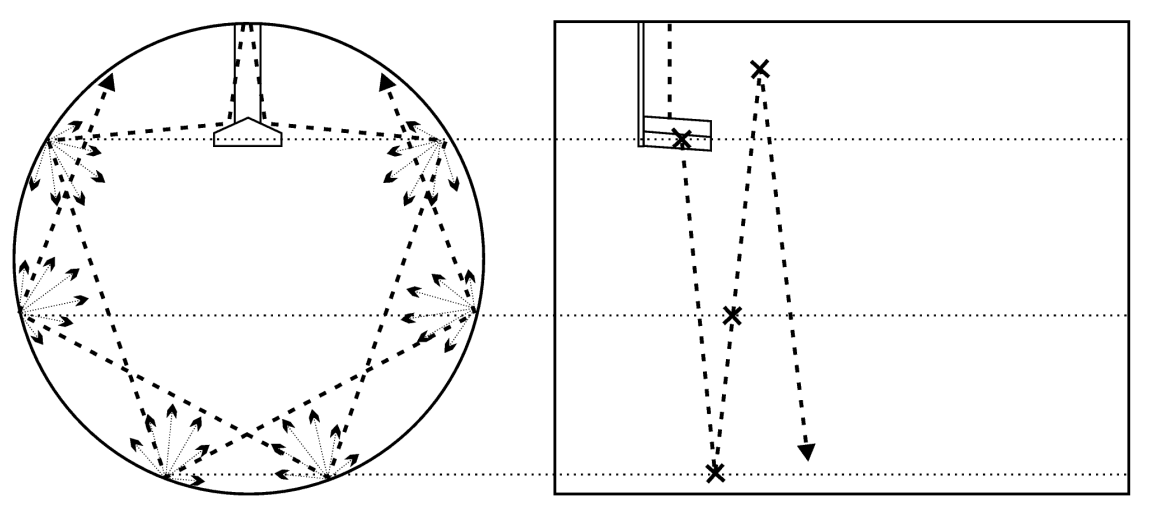

Figure 2: Cross sections through the stray radiation test chamber of W7-X. Millimetre wave radiation is launched from above to a mirror reflecting the radiation to the vessel walls. Due to the wall roughness the radiation is scattered into the central part, generating a power flux density with high isotropy and homogeneity. In the central part the devices under test are positioned [94]. 


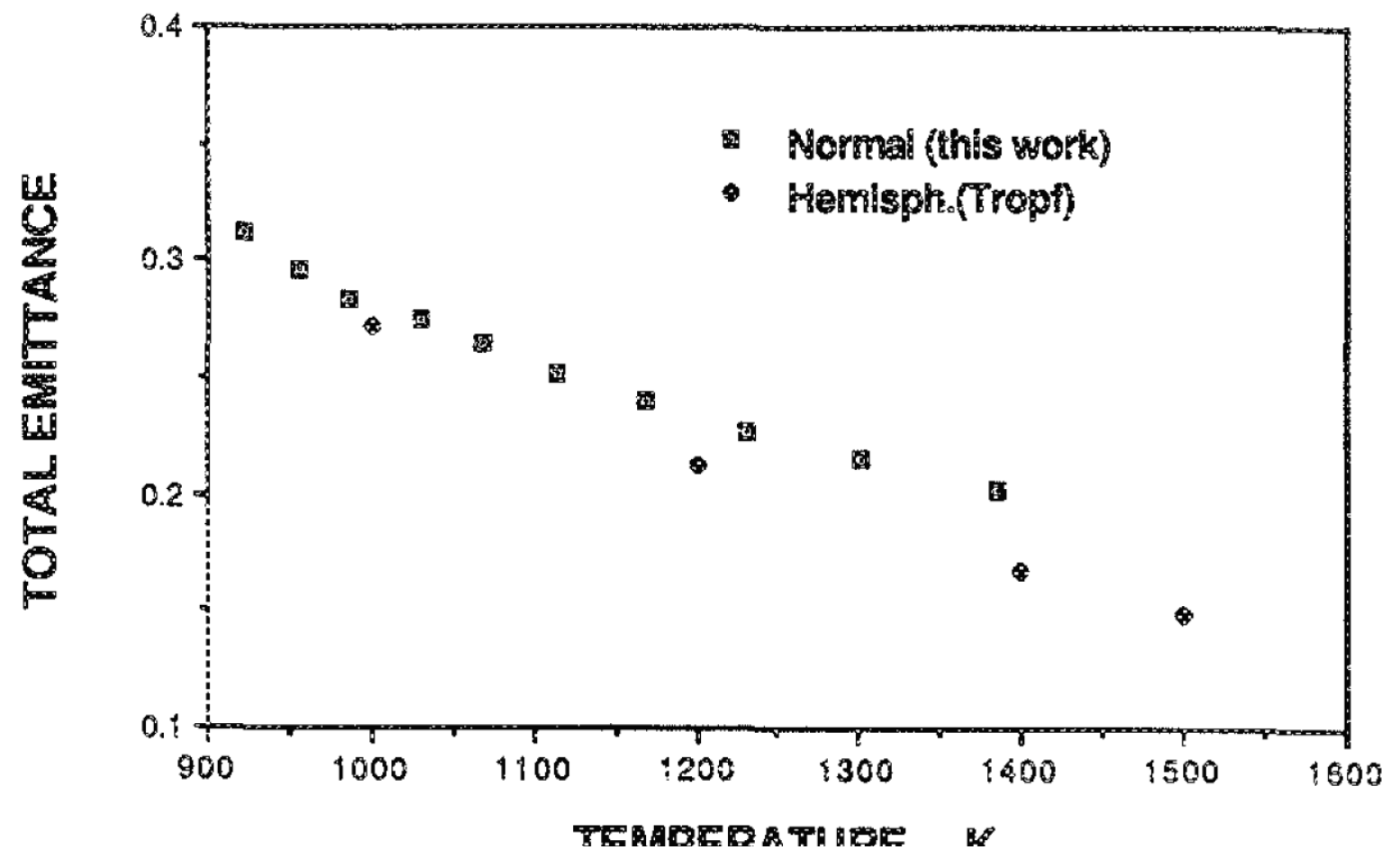

Figure 3: Compilation of total normal [207] and total hemispherical emittance of sapphire. 


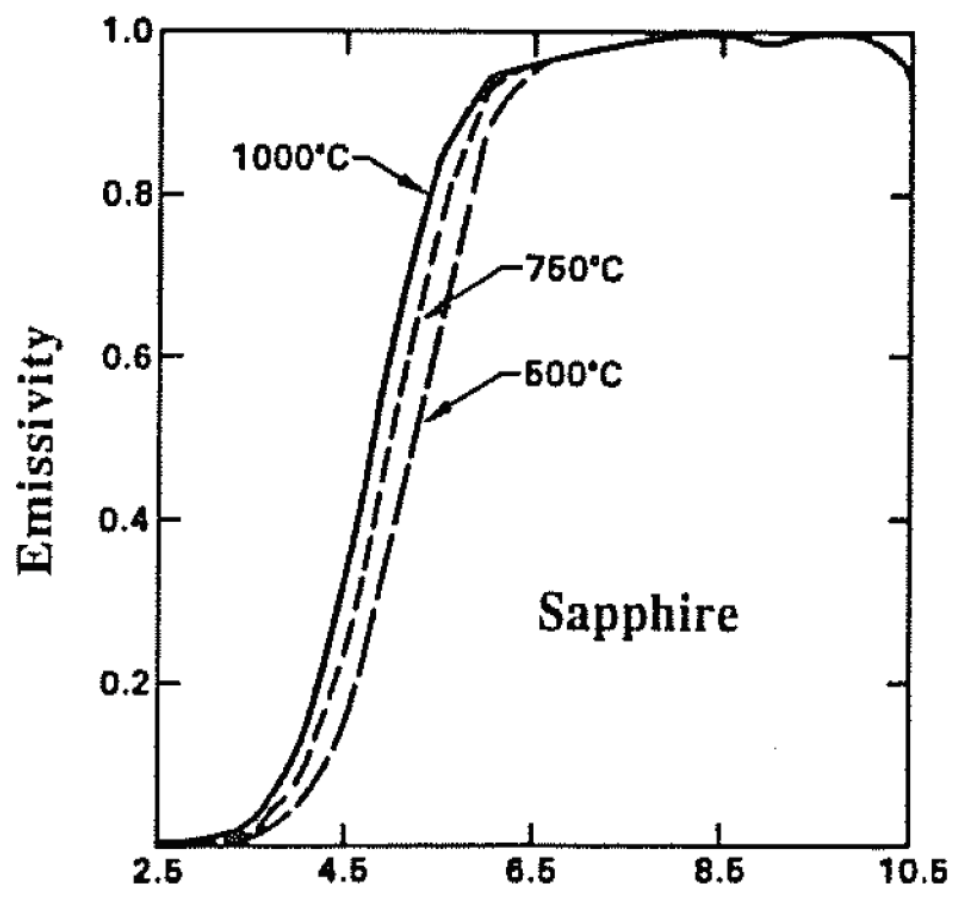

Figure 4: Emissivity of $2 \mathrm{~mm}$ thick sapphire derived from measured normal incidence absorption coefficients [202]. 


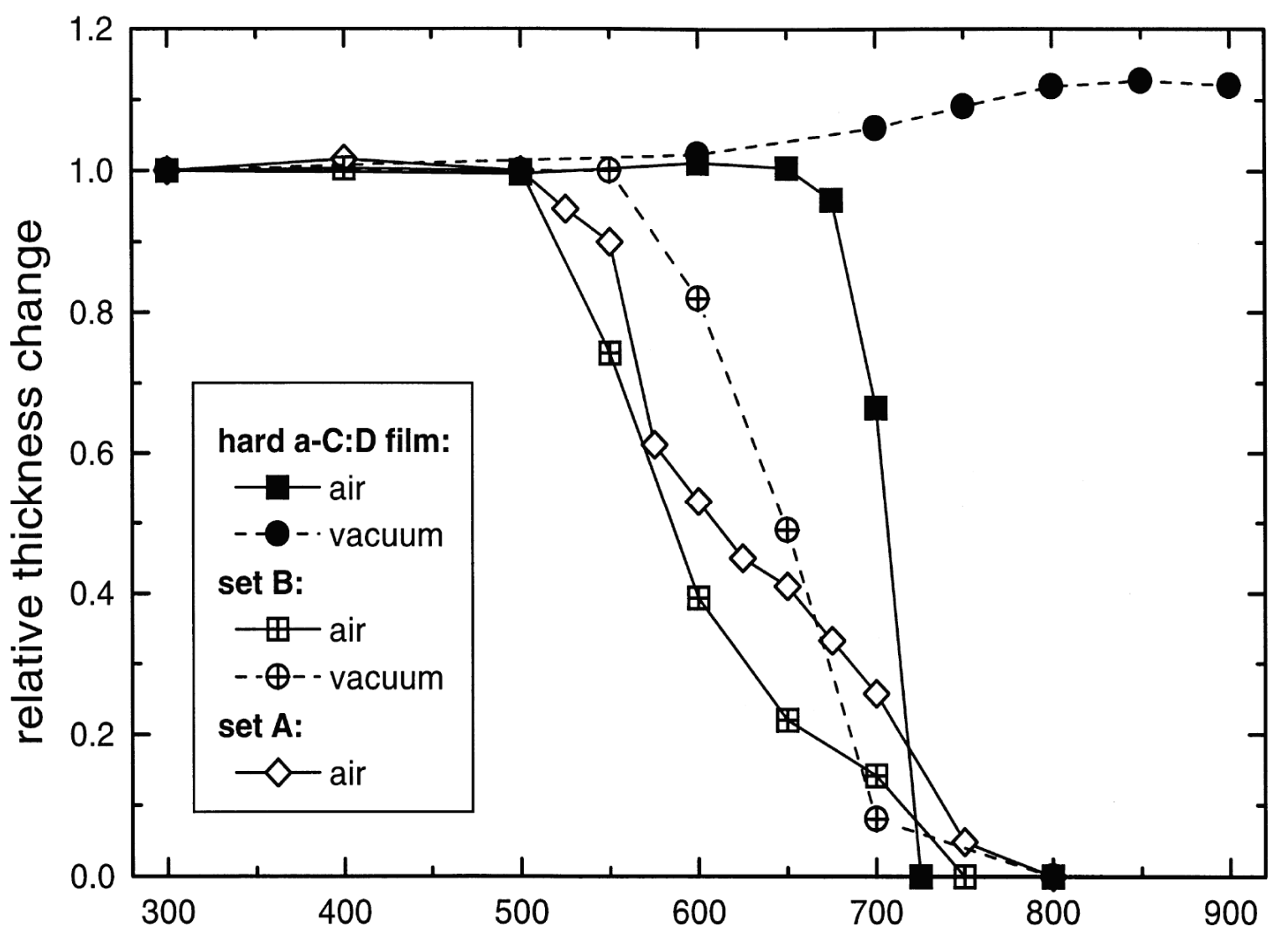

Figure 5: Comparison of the thickness change of soft [241] and hard [247] a-C:D films during annealing for $1 \mathrm{~h}$ in air and vacuum. 


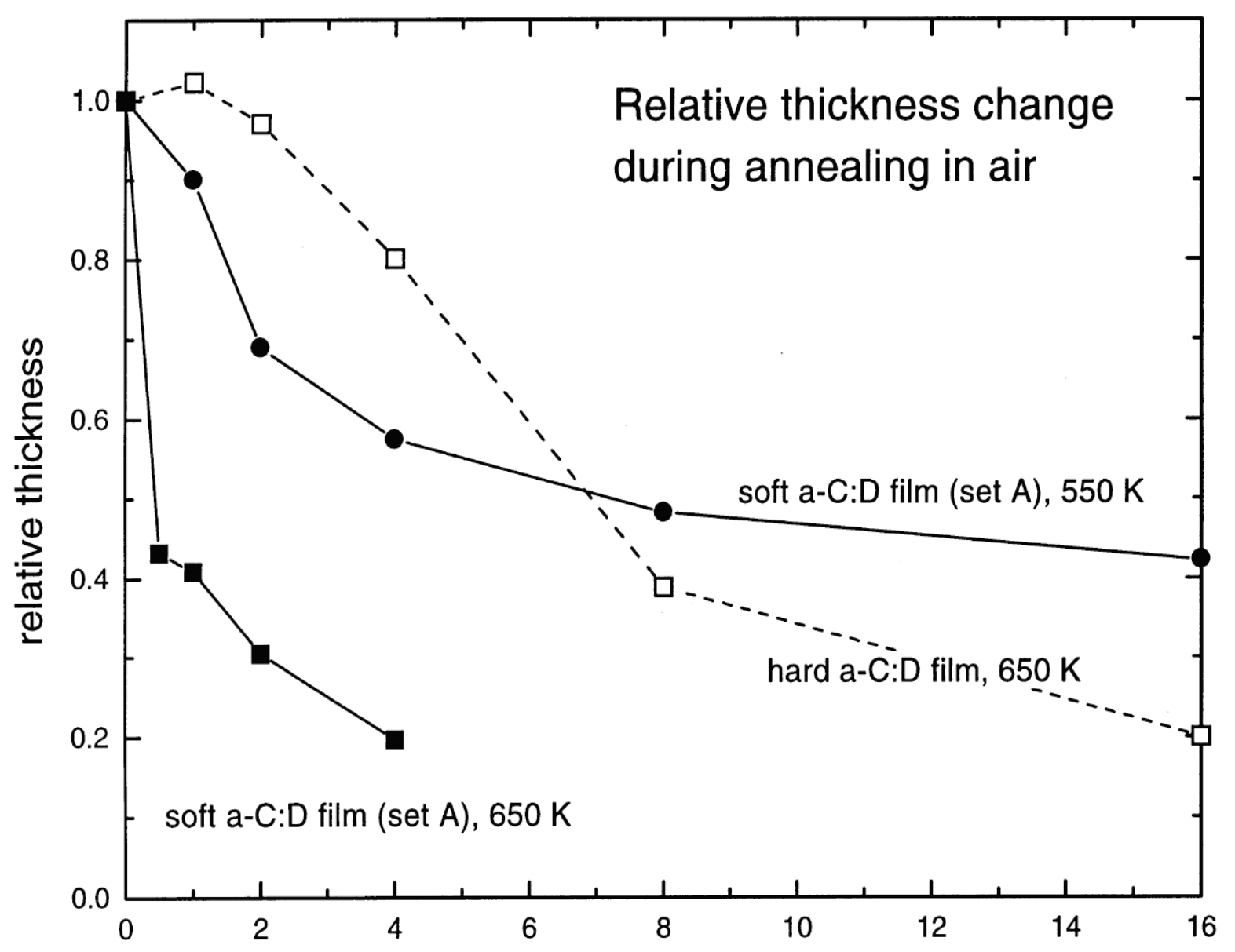

Figure 6: Comparison of the thickness of soft [241] and hard a-C:D [247] films during thermal annealing. 


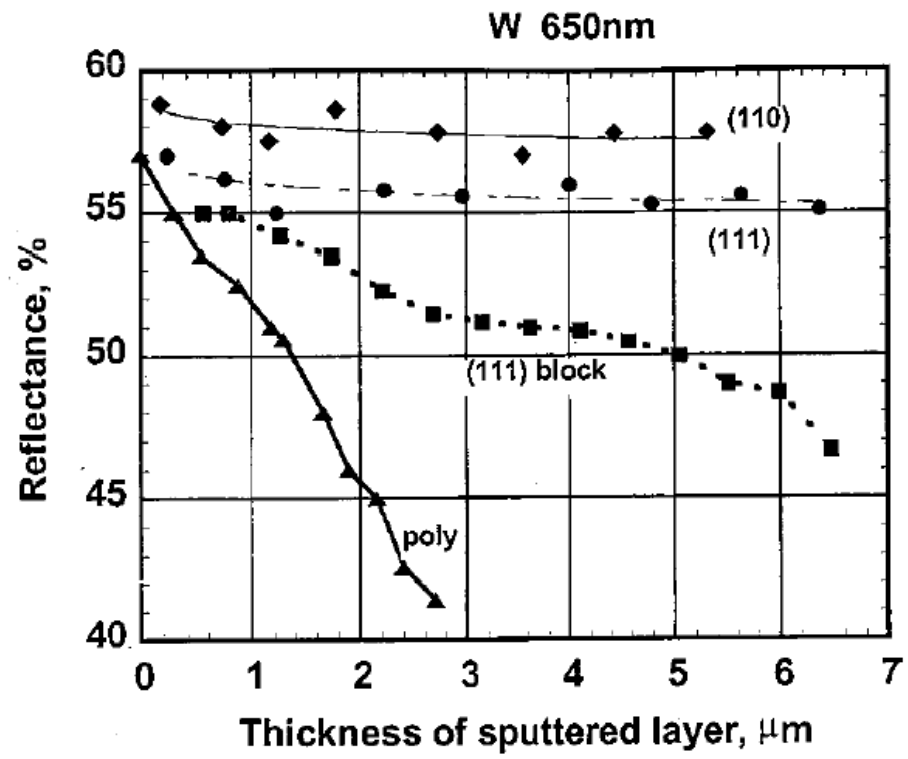

Figure 7: Reflectance change with erosion under ion bombardment measured in the visible spectral range at $650 \mathrm{~nm}$. Only slight changes occur with single crystalline tungsten material compared to polycrystalline one [60]. 


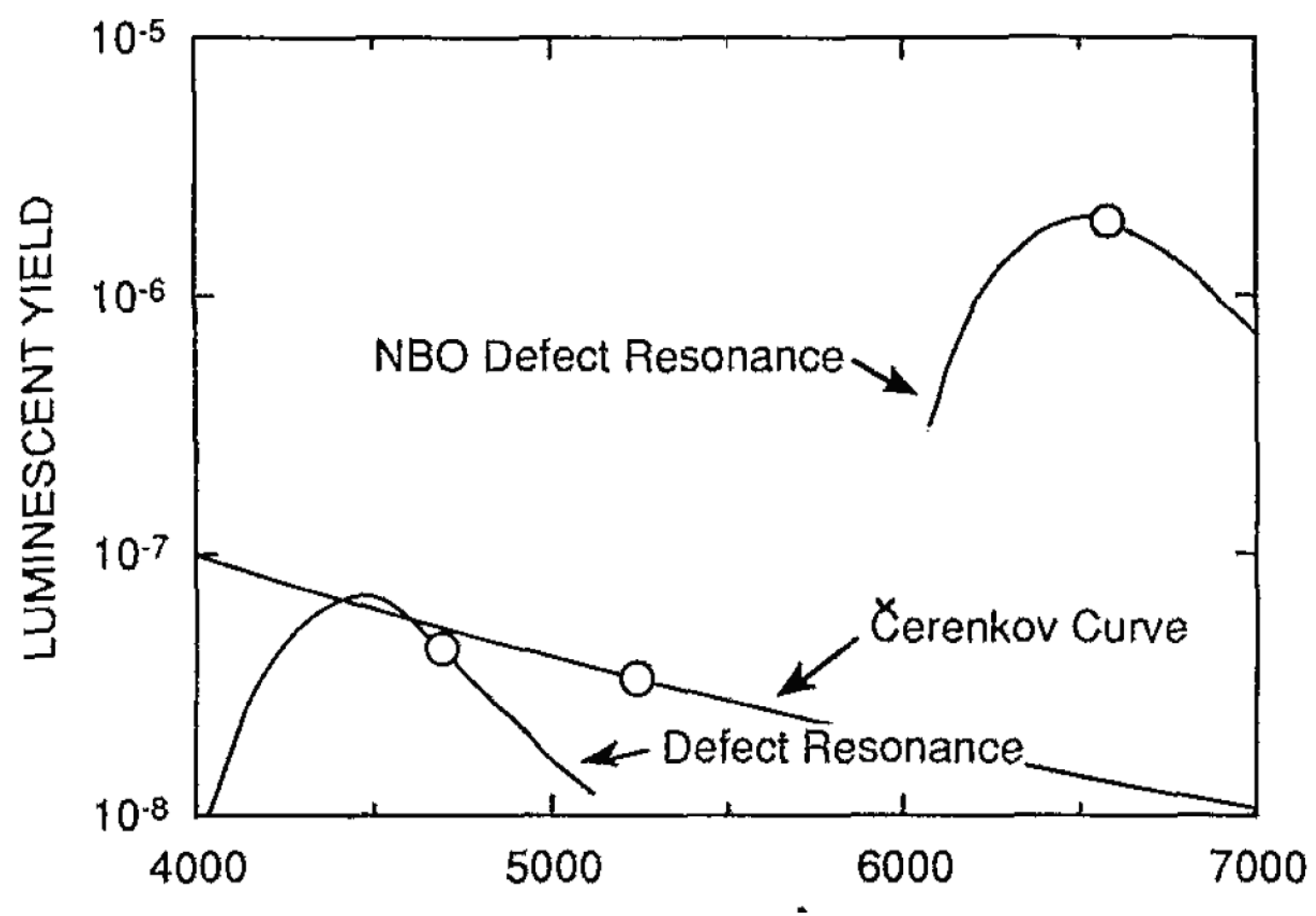

Figure 8: Luminescence in a PCS fibre. The units are luminous surface brightness per neutron emitted by TFTR. The curves are normalised to the points, and are included to show the spectral shape of possible luminescence sources [272]. 


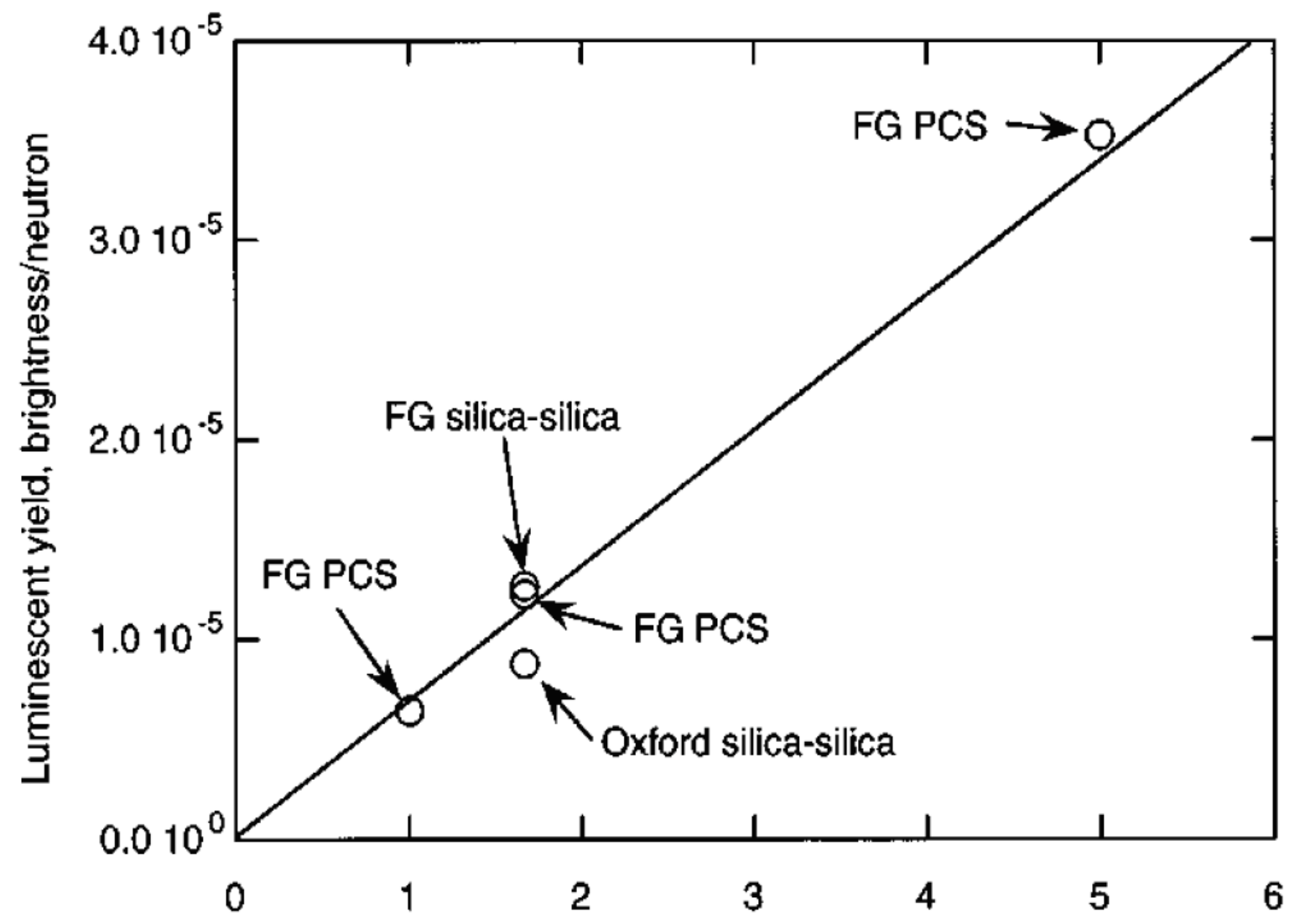

Figure 9: Radioluminescence in the red expressed as equivalent surface brightness and normalised to total neutron production [275]. 


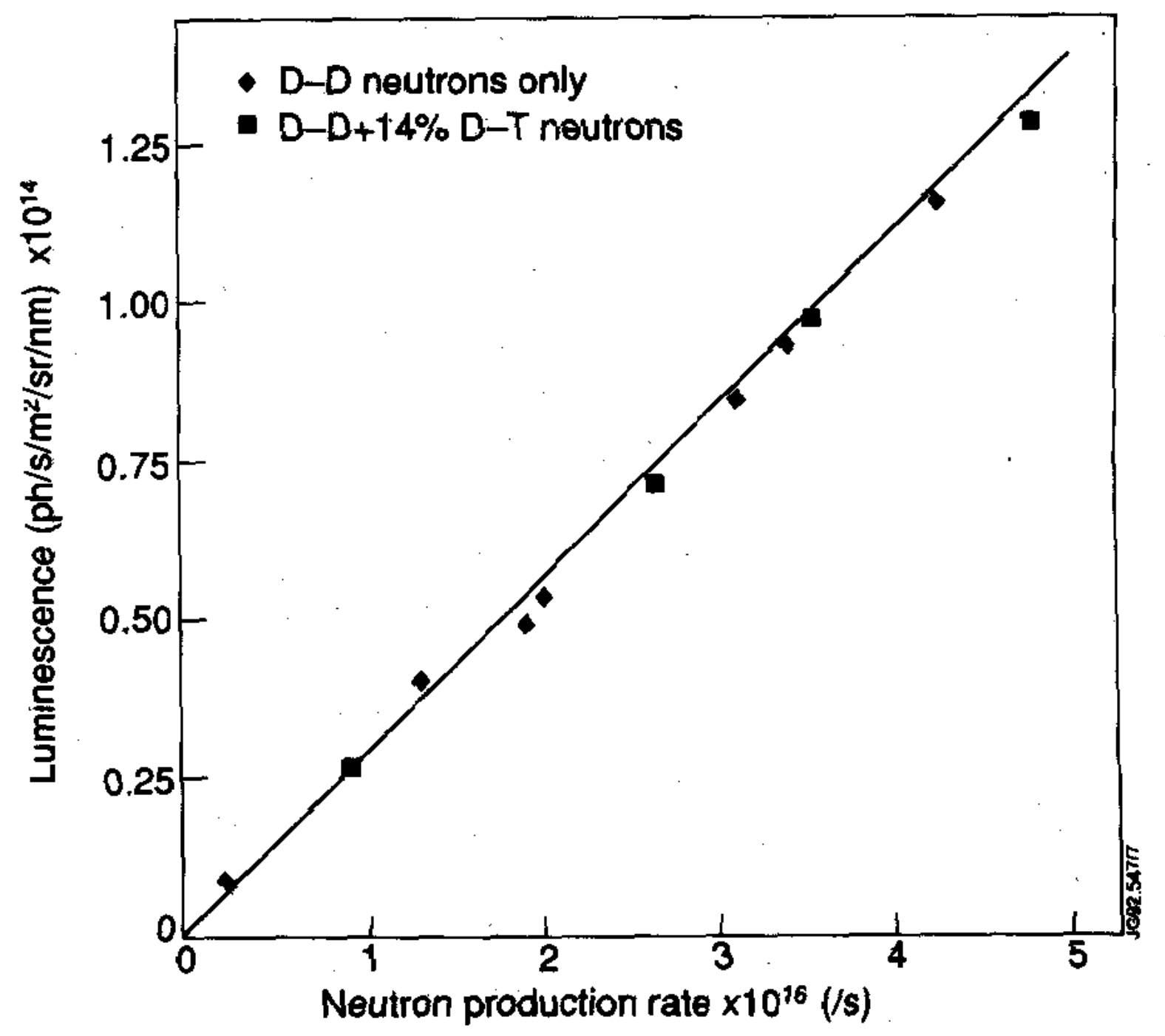

Figure 10: Dependence of luminescence on neutron production rate measured with an interference filter centred at $523.5 \mathrm{~nm}$ (width $1 \mathrm{~nm}$ ). The maximum signal observed in the JET DTE1 experiment corresponds to about one quarter of the continuum intensity measured in another identical channel [273]. 


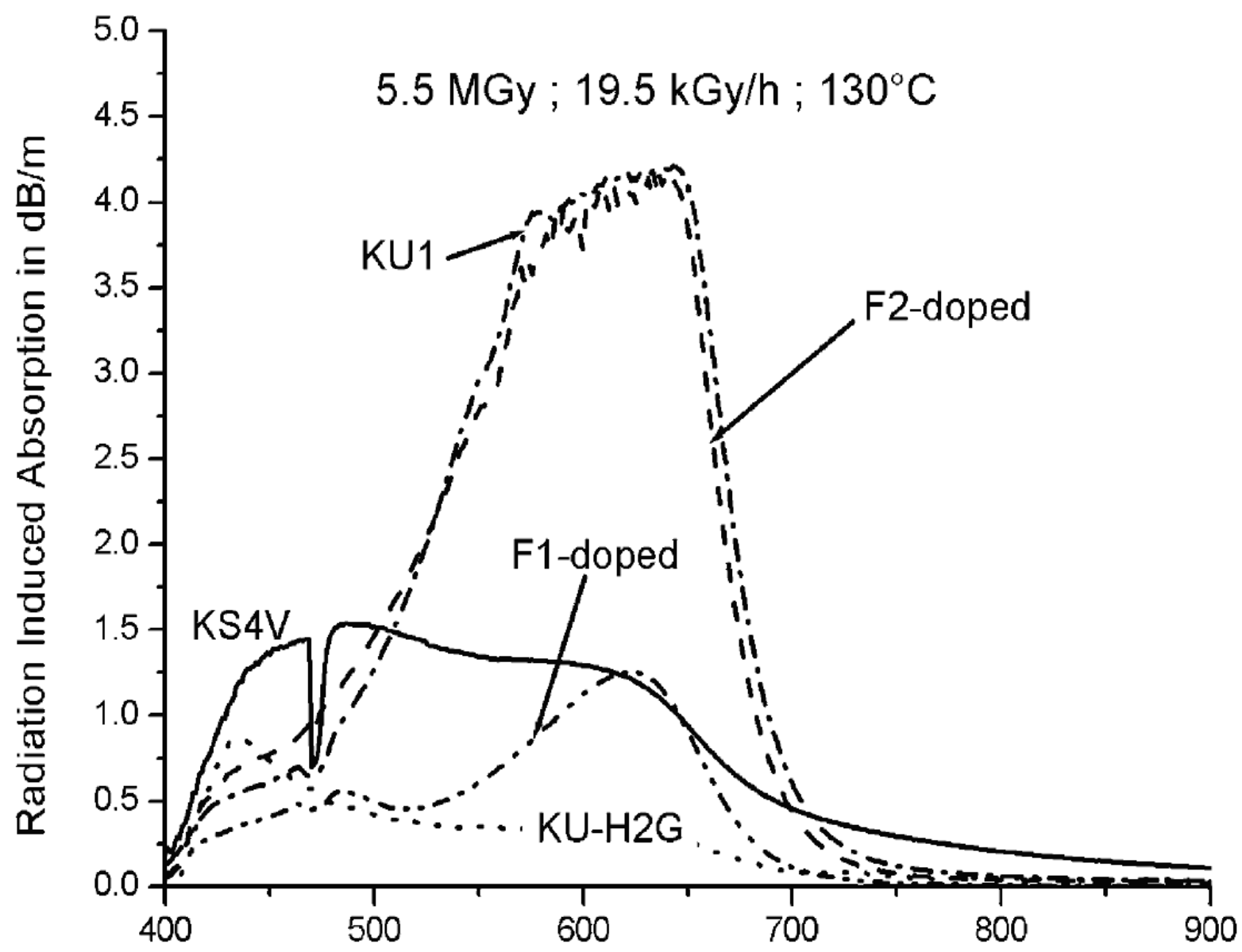

Figure 11: Spectral comparison of the radiation induced absorption due to ${ }^{60} \mathrm{Co}$ irradiation. The lowest absorption is measured in hydrogenated glass (KU-H2G) [173]. 


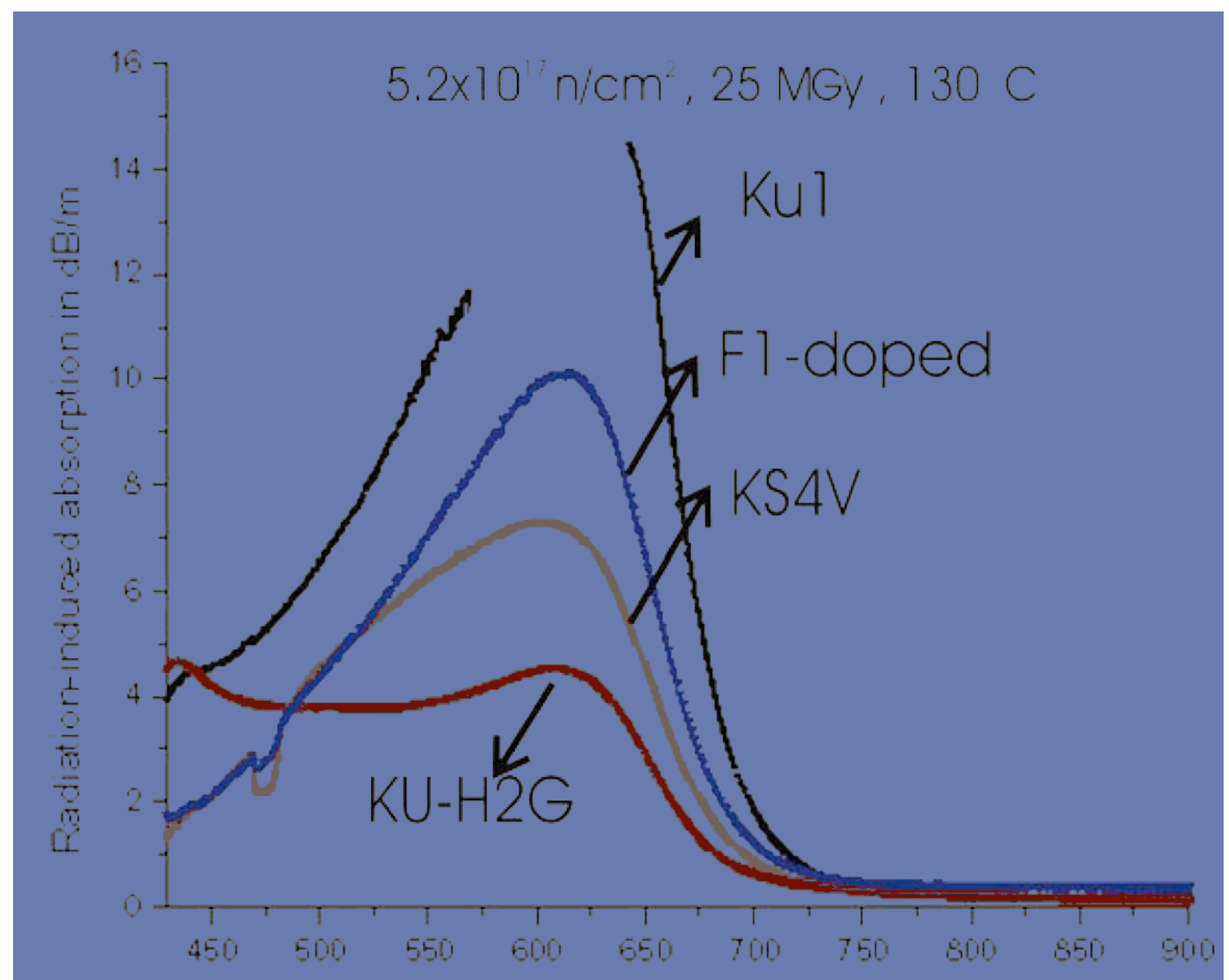

Figure 12: Spectral comparison of radiation induced absorption due to fission reactor irradiation. The lowest absorption is measured in the hydrogen treated material (KU-2HG) [173]. 
a)

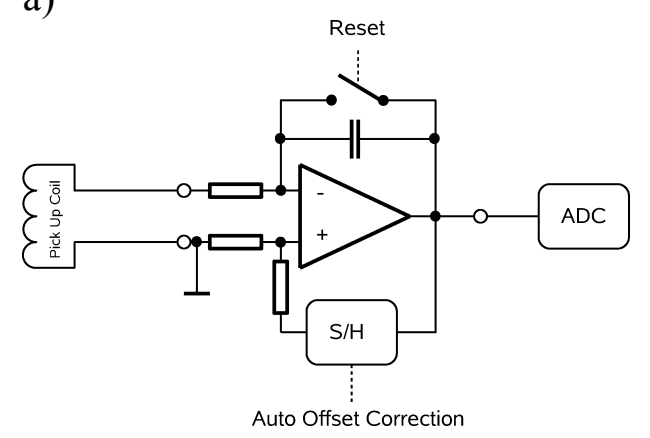

c)

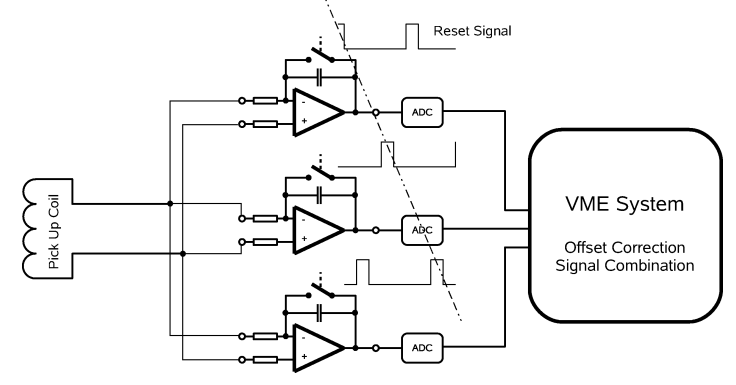

e)

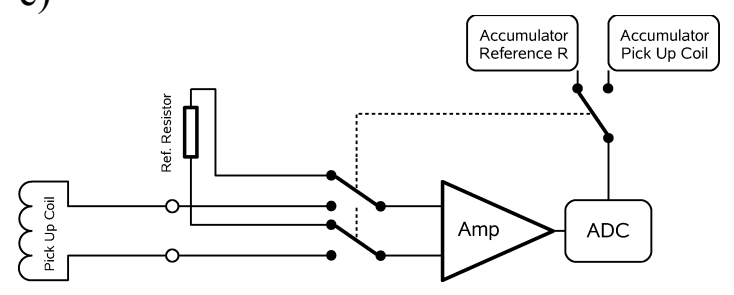

b)

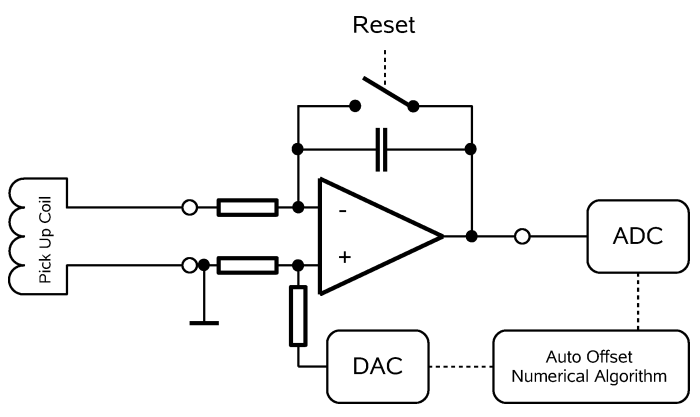

d)

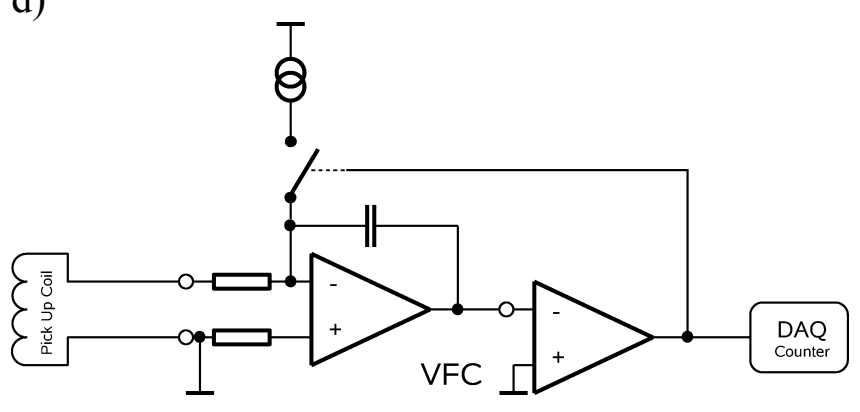

f)

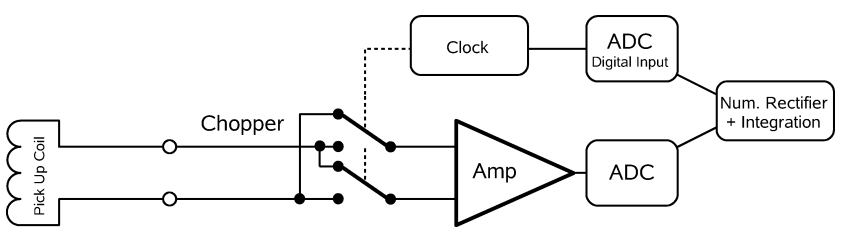

Figure 13: Different types of analogue and digital integrators: a) Analogue integrator with analogue offset correction. b) Analogue integrator with digital offset correction. c) Hybrid analogue and digital integrator with digital offset correction. d) Digital integrator based on voltage to frequency converter, digital offset correction. e) Digital integrator with zero reference. f) Digital chopper integrator, software drift compensation. 